\section{FRANZ FISCHER}

\section{Dinglers Polytechnisches Journal \\ bis zum Tode seines Begründers $(I 820-1855)^{*}$}

Inhalt

Einführung in Problem und Zeit

4.r.4 Sonstige Länder $\ldots \ldots \ldots \ldots \ldots$. . . . . . . . . . . . . . .

x.2 Quellen- und Literaturlage $\ldots \ldots \ldots \ldots$ I03I 4.3 Ubersetzer $\ldots \ldots \ldots \ldots \ldots \ldots$ Ixo8

x.3 Zur Situation der technischen Berichterstattung $\quad 4.4$ Mitarbeiterhonorare . . . . . . . . Irr am Beginn des $x 9$. Jahrhunderts $\ldots \ldots$ ro32

Gründung, Zielsetzung und Herausgeber

des Journals

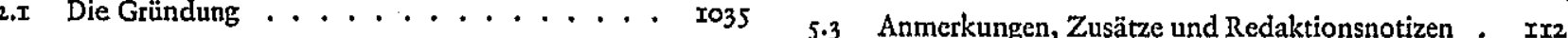

2.2 Die Zielsetzung . . . . . . . . . . . . . r040 6 Preis, Hcrstellungskosten, Auflage und Absatz

2.3 Die Herausgeber $\ldots \ldots \ldots \ldots \ldots$ T043 6.I Preis und Herstellungskosten

2.3.I Johann Gottrried Dingler. . . . . . . . . T043 6.x.x Der Preis des Journals . . . . . . . . . II23

2.3.2 Emil

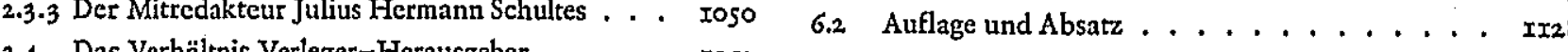

2.4 Das Verhältnis Verleger-Herausgcber . . . . . To52 6.2.x Einschaltung der Behörden zur Absatzstcigerung. II28

Die Grundlagen der Herstellung und das $\quad 6.2 .2$ Werbung in Zeitschriften und Zeitungen . . . . Ir3

Erscheinungsbild des Journals

3.I Das Herausgeberhonorar. . . . . . . . ross

3.2 Die Grundsätze der Schriftleitung . . . . . . . 1060

3.3 Druck und Erscheinungsweise des Journals . . . ro6 Exkurs: Der Zeitungsplan . . . . . . . I067

3.4 Aufmachung und Ausstattung der Zeitschrift

3.4.x Format, Umfang und Zählung. . . . . . . ro7x

3.4.2 Papier und Schrift . . . . . . . . . ro7

3.4.3 Die Tafeln . . . . . . . . . . . . ro ro

3.4.4 Das Register . . . . . . . . . . . ro78

3.5 Der Aufbau des Journals

3.5.x Die Beiträge . . . . . . . . . . xо80

3.5.2 Die Miszellen . . . . . . . . . . . . . ro8

3.5.3 Polytcchnischer Anzeigcr . . . . . . . . . . . .

4 Die Herkunft des Nachrichtenstoffes

4.x Ausgewertete Zeitschriften

4.x.x Englische Zeitschriften. . . . . . . . . x08 $_{5}$

4.r. Französische Zeitschriften . . . . . . . . xo8

4.r.3 Deutsche Zeitschriften . . . . . . . . . xog2

Anhang ............. Ixy Tafel I:

Die Herkunft der Aufsätze im Beitragsteil des Polytechnischen Journals nach den Erscheinungsländern
der ausgewerteten Zeitschriften (einschließlich Monographien und Originalbeiträgen)

Tafel II:

Auflage und Absatz zum Polytcchnischen Journal

8 Quellen- und Litcraturverzechnis . . . . . . Ix37

* Die vorliegende Abhandlung ist dic geringfügig geänderte Hassung einer Hausarbeit zur Prüfung für den höheren Diens Bibliothekathehrinstitut des Köln vorgelegen hat. Gerenüber dem Nordhein-Westtalen in aus Platzgründen zwei Tafeln nicht: Polytechnischen Journal und 2. Die Inhaltsersabließun zum Polytechnischen Journals nach der Dezimalklassifikation.

I Einführung in Problem und Zeit

r.I Aufgabenstellung und Typ der

\section{Zeitschrif}

In der historischen Aufeinanderfolge des Auftretens der Zeitschriftentypen ist die Zeit nach den Napoleonischen Zrien in Dautsond der beginnenden Industrialisierung einherlaufende Aufkommen der technischen Zeitschriften in ihrer ersten, der kommen der technischen Zeitschriften

Diese widmeten sich erstmals vorrangig der Berichterstattung auf dem Gebiet der gesamten mechanischen Technik und sprachen in ihrem Inhalt unter Berïcksichtigung der mathematischen und naturwissenschaftlichen Grundlagen alle diejenigen Kenntnisse und Fertigkeiten an, welche zur gehr , we whe zur Gewerbe und Kunste thr notwendig gehalten wurden Ihre publizistische Wirksamkeit gründete aber vor allem darin, daß sie sich in gemeinfaßlicher Sprache und durch ihren breiten polytechnischen Themenkatalog an den gesamten Kreis der an anwendungsbezogener Technil Interessierten wandten.

Eine der ältesten und erfolgreichsten Zeitschriften dieser neuen Gatung der technischen Berichterstattun in iluer polytechishen tor war dos Polytechische in ilher polytechischen Journal. Es wurde seit I820 von dem Augsburger Che miker und Fabrikanten Johann Gottfried Dingler ( $177^{8}$ bis 1855 ) herausgegeben und von der J. G. Cotta'schen Buchhandlung in Stuttgart verlegt. Der Werdegang dieser Zeitschrift soll hier bis zum Tode ihres ersten Herausgebers und Begründers Johann Gottfried Dingler im Jahre 1855 , das heißt, bis zum Abschluß des 36. JahrIn wangs unden.

Aufgabe der vorliegenden Untersuchung ist es, die sachlichen und personellen, die organisatorischen und wirtschaftlichen Grundlagen und Aktivitäten von Redaktion und Verlag im Zusammenhang mit der Herausgab sowie die Gestaltung von Form und Inhalt der Zeitschrift aufzuzeigen. Um nicht selbstgenügsam in der Isolierung des Einzelfalls zu verharren, wird darüber hinaus versucht den allgemeinen zeitlichen Rahmen und Wardel der technischen Berichterstattung mit der
des Polytechnischen Journals zu verbinden.

${ }^{1}$ In Allgemeine deutsche Real-Encyklopädie für die gebildeten Stände. Converstions-Lexikon I2 (Leipzig: Brockhaus IO. Auf x854), S. 256 werden die Ziele der polytechnischen Journale wie folgt umrissen: "Polytechnische Journale wollen nicht blos alle Richtungen der Technik umfassen, sondern auch der reine Empirik gegeniiber das wissenschaftliche Element festhalten.
Das durch den Tod des Begründers und ersten Herausgebers J. G. Dingler gewonnene zufällige Abgrenzungsdatum 2855 bedeutet keinc einschneidende Zäsur im Sinne einer neuen proklamatischen Zielsetzung oder einer wesentlichen Veränderung im Stil des Polytechnischen Journals. Diese Abgrenzung erhält aber doch einen tieferen Sinn aus der allgemeinen Wirtschafts- und Technikgeschichte und den mit ihr in Zusammenhang stehenden Veränderungen der technischen periodischen Fachpresse dieser Jahre um kurz nach der Mitte des I9. Jahrhunderts.

In diesen Jahren ist der entscheidende Durchbruch von der Frühindustrialisierung zu den späteren industriellen liserus Answeitung und Verselbständigung der technichen Einzeldisziplinen untereinander sowie von anderen Wissensbereichen, dann dic Herausbildung und der Bedeutungsanstieg eines bereits theoretisch an technischen Schulen ausgebildeten Technikerberufsstandes mit qualifizierten Literaturwünschen hatten überdies - schon vor der Jahrhundertmitte erkennbar - um etwa $x 850$ das Dende der Ain der Vorherrschaft der Zeitschriften des polytechnischen Charakters zur Folge. Es entstanden

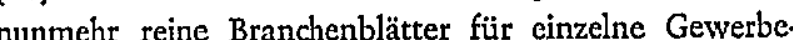
und Industriezwcige mit oft weitgefächerter Thematik, berufsständisch orientierte Zeitschriften zur Interessenvertretung und fachlichen Unterrichtung der Berufsgruppen oder rein ingenieurtechnische Zeitschriften. Ihre Lektüre verlangte technische Vorbildung und theoretische Schulung. Diese Zcitschriften führten in Verbindung mi dem nunmelr spürbar auflebenden Vereinswesen ab de Jahrhundertmitte den einzelnen Berufsangehörigen aus seiner individuellen oder "örtlichen. Isolation in eine von fachlichen Bildungsinteressen oder berufsständischen Zielsetzungen geformte Gemeinschaft" hinein².

Jedenfalls ist mit der Untersuchung von Dinglers Polytechnischem Journal im Zeitraum I820-I855 der publizistisch, bildungs- und technikgeschichtlich relevanteste Abschnitt dieses Zeitschriftentyps erfaßt, in welchem dieser Art der technischen Berichterstattung im Vormär and der Fring und der Frühindustrillsienng in erster Linie die Mittle kunktio 2 Hans-Joachim Hamann: Dic ökonomischen und techni-
schen Z Zeitschriften in der Frühzeit der deutschen Industrie. Mschr. Dipl.arbeit Seminar f. Wirtschafts- und Sozialgeschichte Univ. Köln (Köln 1957/58), S. I05. 
Das Polytechnische Journal selbst erschien allerdings noch bis in das erste Drittel unseres Jahrhunderts. Nach Tode Der Emil Maximilion Dingler (r806-r874), führte die Zeitschrift seit Band 2ra (Jg. 1874) den Namen "Dinglers Polytechnisches Journal«. Die Schriftleitung des Journals ging damals auch aus den Händen von Praktikern in die von Dozenten und Professoren an Technischen Hochschulen über. 1897 ist dic Zeitschrift aus dem Verlag Cotta an Arnold Bergsträsser in Stuttgart übergegangen. Seit 1902 wurde dos Journal in Berlin durch die Verlagsuch andlung $R$ thard Dinge vahrgang und 346 and fel sic dann Igs i den damaligen wirtschaftlichen btheit dieses Zeitschriftentyps zum Opfer.

I.2 Quellen- und Literaturlage

Eine Hauptquelle dieser Arbeit ist die Zeitschrift selbs Aufmachung und Ausstattung Autorennamen, Artikel nachweise und Inhalt sowie redaktionelle Anmerkungen der Notizen sind zentralc authentische historische Quellen, die Wesentliches zur Geschichte der Zeitschrift aus zusagen vermögen.

An ungedruckten Quellen konnten aus dem CottaArchiv (Stiftung der Stuttgarter Zeitung) des SchillerNationalmuseums in Marbach/Neckar vor allem 363 Briefe der Herausgeber Johann Gottried und Emil Maximilian Dingler an Cotta aus dem Zeitraum I8I7 bis 1874 herangezogen werden. Diese Briefe vermittelten zahlreiche wertvolle Erkenntnisse und Einsichten. Sie tragen allerdings gelegentlich nur Notizen- oder Anweisungscharakter in Zettelform und waren insgesamt nicht so dicht und nicht durchwegs so ergiebig, wie man es sich gewünscht hätte. Manche Probleme sind in ihnen nur punktuell oder in unzusammenhängenden Details angesprochen, wie andererseits besonders im Anfangsjhrzehnt allgemeine nicht das Polytechnische Joun etreffende Frater

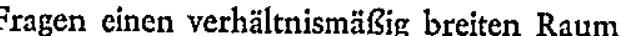
einnehmen. Vor allem aber fehlen die Bricfe Cottas an die Herausgeber. Aufgeworfene Probleme können daher

${ }^{3}$ W. Dietze: Aus der Geschichte des Journals. Dinglers olytechn. Journal I820-T20. Festschrift zum hundertiährier Bestchen der Zeitschrift (Berlin 1920), S. 8; Liselotte Lohrer: Cotta. Geschichte eines Verlages 1659-X959 (Stuttgart x959), S. I5o, gibt an, daß Dinglers Polyt. Journal bereits 1896 an Richard Dictze abgegeben worden ist; Wilhelm Treuc: Din eitschrift. echnik-Geschichte 37 (Dïsseldorf I970), S. I2. nur aus der Sicht der Antwortbriefe Dinglers ohne genaue Kenntnis des Inhalts der Schreiben Cottas an die beiden Dingler beurteilt werden. Auf eine Auswertun der unter Ceorg von Cotn im Verlaghous Cota Die darin enthaltenen Auszüge der aus dem Verlag haus Cotta ausgehenden Post sind zu kurz, um aus ihnen wesentliche Aussagen gewinnen zu können.

Literatur zum Polytechnischen Journal, welche über zeitgenössische Würdigungen oder Erwähnungen in Da stellungen zur Geschichte des Zeitschriftenwesens hinder Geschichte des Journalsu, den der damalige Verleger V. Dietze in der Festschrift zum hundertijhirigen Bestehen der Zeitschrift I920 verfaßt hat ${ }^{3}$.

Die verstärkte Beschäftigung mit neuerer Wirtschaftsund Technikgeschichte in Deutschland im letzten Vierteljahrhundert hat kurz hintereinander $x 969$ und $\times 970 \mathrm{zwC}$ Vertreter dieses Faches veranlaßt, in zum Teil programmatischer Weise auf die Bedeutung der polytechnische die Technik- und Industrialisierungsceschichte im allge meinen sowie auf die Wichtigkeit von Dinglers Polytechnischem Journal im besonderen hinzuweisent. Einer de beiden Propagatoren der Zeitschrift, Prof. Wilhelm Treu vom Historischen Seminar der Technischen Universitär Hannover, ist gemeinsam mit Karl-Heinz Manegold auch Herausgeber der "Documenta Technica. Darstellungen und Quellen zur Technikgeschichte", in deren Reihe Zeit YZim-Ne York, in Reprine heraugegeben wird. Nach dem Ve lagskatalog "Zeitschriften I974" und schriftlicher Mi teilung des Verlags Georg Olms sind bisher dic Bände I-xo lieferbar. Die Reproduktion der weiteren Bände wird in Microform weitergeführt.

I.3 Zur Situation der technischen

Berichterstattung am Beginn des

I9. Jahrhunderts

Die Anfänge einer regelmäßigen Nachrichtenvermittlun auf technischem Gebiet bcgegnen in Deutschland bereits im zweiten Viertel des $x 8$. Jahrhunderts in den vorwio

4 Ulrich Troitzsch: Zur Entwicklung der (poly-) technischen Zeitschriften in Deutschland zwischen 1820 und $x 850$. Wissen schaft, Wirtschaft und Technik. Studien zur Geschichte. Wihelm Treuc zum 60. Geburtstag. Hrsg. von Karl-Hcinz Mane gold (München x969), S. 331-339; W. Treuc: Dinglers Poly- gend ökonomisch-staatswissenschaftlich ausgerichteten kameralistischen Zeitschriften. Die Begründung des akademischen Lehrfachs Technologic im Rahmen der Staatswissenschaften durch den Göttinger Professor der Okonomie Johann Beckmann (I739-I8II) und das Aufkommen der kamerastisch-technologischen Zeitschriften in Dite Gewichte in der Berichterstattung nicht grundlegend Wie Beckmann blieben auch dic kameralistisch-technologischen Zeitschriften im enzyklopädischen Sinne der Zeit einer beschreibenden Gewerbekunde verhaftet, wclche Technik und Wirtschaft mit Staatskunde und Gesellschaftslehre verklammerte.

Entsprechend der geringen Bedeutung der Maschine im deutschen Gewerbelcben war vor allem dic mechansche Technik untervertreten ${ }^{5}$.

Erst in der Folge hat das Zusammenwirken verschiodener Faktoren die Gründung und die Lebensfähigkei der polytechnischen Zeitschriften begünstigt:

I) Führte die Wirtschaftslehre von Adam Smith vom wohl verstandenen Selbstinteresse als grundlegende Kraft der wirtschaftlichen Entwicklung in einer wirtschaftspolitisch möglichst staatsfreien Gesellschaft sowic die gesamte politisch-geistige Umwälzung der Zeit zur Aulös Auflosung jener au die oberste Lenkung und Ordnung der Wirtschaft vorbehalten hattc. Unter bewußter Aktivierung der Kräfte des mitwirkenden Bürgertums ging es der Gewerbeförderung des heraufkommenden individualistischen und liberalen Zeitalters in der ersten Hälfte des 19 . Jahrhunderts um die Erweckung und Förderung der Initiative der Unternehmer sowic um die Pflege des Geistes der Selbsthilfe und

"Siehe dic umfassende, zu der Zeit des Erscheinens des Lexikons cigentlich schon überholte Definition und Aufgabenbeschreibung der Technologie in: Das großsc ConversationLexikon thr die gebildeten Stando. Has. Von Josef Meyer, 4 berts a cer Technologie in der nhezu gleichcelts (Bir die ro. Auldeten Stände (Brockhaus) I4, S. 7 rgf. Dic cngere Begriffserklärung "Technologicu bereits in der Auflage de Brockhaus von I817: Conversations-Lexikon oder encyklop disches Handwörterbuch für gebildete Stände 9 (Leipzi Altenburg: Brockhaus, $x 8 \mathrm{I}_{7}$ ), S. 709. Zum übrigen siehe: Ku Schulz: Um dic Zukunft der technischen Fachpresse. Entwicklung und Gegenwartsprobleme der technischen Berichterstatung in Deutschland (Berlin X93r), S. I7ff.; Karl Karmarsch. Geschichte der Technologic seit der Mitte des Is. Jahrhunderts (Micse sillo a der Gemeinnützigkeit. Polytechnische Schulen, polytechnische oder Gewerbevercine, Zeitschriften, Patentschutz als Anregung für den Erfindungsgeist und Ausstellungen wurden nun dic Hauptmittel der von der zuständigen Ressortbürokratie staatlicherseits meist höchst energisch cförderten Erziehung zur Industrie ${ }^{6}$.

2) Erfuhr der Gebrauch des Begriffs allgemeine Technologie mit seiner Herauslösung aus der kameralistischen etrachtungswcise einer im funktollon Zusammenang der einzelnen Wirtschafts we gesehen Technik allmählich eine Einengung auf das Gebiet der chemischen und mechanischen Technologie im Sinne einer reinen Verfahrenskunde. Der neuc Name Polytechnik wurde hingegen zum »Inbegriff aller zur Ausübung der verschiedenen Künste und Gewerbe erforderlichen Kenntnisse und Geschicklichkeiten, meist mit dem Nebenbe-

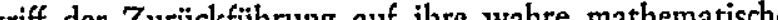
the nun, zunehmend losglost von ihrem staatlichen oder gesellschaftlichen Bezug, gesehen um ihrer selbst willen bzw. in ihrem unmittelbaren Anwendungsbezug. Dic eine Schulgattung begründende und den Namen Polytechnik popularisierende Gründung der Pariser "Êcole polytechnique" in der Französischen Revolution I795 will denn auch nur mehr »umfassende technische Kenntnisse im Hinblick auf eine praktische Anwendung vermitreln $\ll^{8}$

3) Hatte schon ausgangs des I8. Jahrhunderts der industrielle Aufschwung Englands und von da sich fortpllanzend besonders in Frankreich und Belgien das Interesse an dessen technischen Grundlagen geweckt. De Leistungsfähigkeit des maschineneinsetzenden englischen Fabrikenwesens war man sich nach der durch Kontinentalsperre und Kriege verursachten Absperrung in de Uherchwemmung des deutschen Marktes mit englischen in den ersten Nachkriegsjahren nach 8x5 neuerdings schlagartig und schmerzlich in Deutschland bcwußt geworden. Und »es ist gar nicht zu leugnen, $\mathrm{da} B$ die ungeheuere Entwickelung der Industrie in England den Anstoß zur Entwickelung der Polytechnik au dem Continente ... gegeben hat ${ }^{7}$. Der wiederhergestellt Friedenszustand erleichterte überdies nach r8Is die lite-

Siehe auch Franz Schnabel: Deutschc Geschichte im neunnik (Freiburg/Breisgau, 1934).S. 292 ff., 3 roff.

' Albrecht Timm: Kleine Geschichte der Technologie (Urban it. Eing, Suschiche 
rarische Verbindung Deutschlands mit England und auch Frankreich 0

4) Bildete die ausgedehnte, bcreits entwickelte naturwissenschaftliche und technische Zeitschriftenliteratur Englands und Frankreichs ein Vorbild sowie eine Herausforderung zur Nachahmung ${ }^{10}$. Thre Existemz stellee cine zentrale Voraussetzung für dic Nachrichtenbeschaffung eines $\mathrm{zu}$ gründenden deutschen technischen Organs dar das der inlandischen Rückständigkeit unter anderem durch Vernittlung der neuesten technischen Kenntnisse

des Auslandes abhelfen wollte und das nur in Grenzen auf qualifizierte einheimische Mitarbeiter zurückgreife konnte. Neben den schon existierenden naturwissenschaftlichen und den nahezu gleichzeitig aufkommenden gewerblichen und polytechnischen Periodika in Deutschdeten Technikerstandes wuchsen aber einem Blatt von der Art des Polytechnischen Journals auch im Inland in Laufe der Jahrc zunchmend Nachrichtenquellen, Beiträger und Leser $\mathrm{zu}$.

\section{Gründung, Zielsetzung und Herausgeber des Journals}

\section{2.x Die Gründung}

Die Initiative zur Gründung des Polytechnischen Journals ging von Johann Gottfricd Dingler aus

Am 27. Juni $x 8 x 9$ wandte er sich brieflich an den bereits renommierten Verleger Johann Friedrich von Cotta (I764-x832) in Stuttgart und teilte ihm mit, daß er sich entschließen würde, ab Jänner I820 eine polytechnisch Zeitschrift in monatlichen Heften herauszugeben, »wen sich mit dem Verlage ein dem Unternehmen gewachsene Verleger befassen würde«. Als Begrïndung für die Herausgabe der Zeitschrift gab er erläuternd an, $d a \beta$ noch un keiner Zeit sdas Bedürfnis zur Verall ameinuß no echnischer Kenntnisse als in anchann

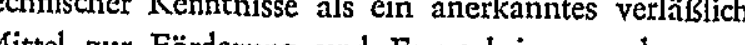
der vate andischen Industrie und Nazionalvermögens mehr gefühlt als jetzt« wurde. Befleißigten sich doch gerade "diejenigen Regierungen, welche im Wohlstande ihrer Unterthanen auch den ihrigen gegtündet sehen, um polytechnische Schulen, Institute und Vereine zu gründen und durch sie den herabgekommenen Wohlstand der Indu stric- und Gewerbs-Classen herbcizuführen «. Zur Erreichung diescr Zwecke dürfte seiner Ansicht Zurh EnGut redigirte polytechnische $Z$ eitshif do fordellds Mittel sem Dinger verich Cotta noch über die Tendenz und die Art der Bericht-

- Karmarsch: Geschichte, S. 893; Schulz: Fachpresse, S. 23 f. ur Lage der Wittschaft in Deutschland siche auch Johan Gottfried Dingler: Polyt. Journal 4 (I82x), S. 486 "Zur Zeit Wer wontinentalsperre effreute sich die deu Ve Industric manigfaltiger Vortheile vorzüglich durch die

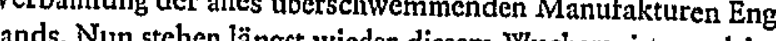
durch leichte Preise den Küuer dicsem Wuchergeiste, welch Gärktc Deutschlands offen.

${ }^{10} \mathrm{~W}$. Treue: Dinglers Polytechnisches Journal, S. 4 erstatung der Zeitschrift Ḧber dio bisher erschionenen Blätter ähnlicher Art, die keineswegs den von ihm gestellten polytechnischen Anforderungen entsprächen, und geht dann zielstrebig über zur technischen und geschäftlichen Seite dieser Gründung. Jeden Monat sollte wein Heft zu 7 bis 8 Bogen in gr. $8^{\circ}$, mit Kupfern begleite erscheinen " Als Honorar forderte er 2500 Gulden iti lich, wovon er aber die zur Auswertung notwendige inund ausländische Literatur herbeizuschaffen und wyon Zeit zu Zeit einige unumgänglich nothwendige Versuch und Prüfungen empfohlener Gegenstände" zu veranstalten hätte, so daß ihm seinem Uberschlage nach mnoch kein anderer Gewinn zu Theil würde als der Ehre und das Bewußtsein etwas bleibendes Gemeinnützige gegründet zu haben «. Dingler schloß seinen Brief, Won diesem Vorhaben setze ich hiemit Ew. Hochwohl Vorerst in Kenntnis, und wenn sich Dieselben zum Verlage dieses verdienstlichen Unternehmen geneig erklären, so werde ich Ew. pp. einen speziellern Contrac einzusenden die Ehre haben.

Dieses "Gründungsschreiben« verrät schon durch seinen bestimmten und klaren Ton einen mit dem Stand der Wirtschaft und Wirtschaftsförderung, der technischen Literatur sowic der Zeitschriftenredaltion und dem Vet Th vertrauten Mann. Und in der Tat hat Dingler, an die Giundung des Polyter schen Journals schritt, zum Teil allein, zum Teil in Gemeinschaft mit anderen bereits seit $x 806$ Fortsetzungswerke von zwei bis vie Bänden, vornehmlich zur Textildruckerei und Färberei herausgegeben. $x 8 x 7 / x 8$ ist von ihm gemeinsam mi W. H. von Kurrer ein englisches Färbebuch in Übersetzungen exschienen, welches er mit Anmirkungen und Zusätzen versehen hatte. $x 8 \mathrm{r} 8$ hat or überdies auf ${ }^{11} \mathrm{CA} \mathrm{I/427.} \mathrm{Juni} x 8 \mathrm{xg}$ und W. Dietze: Geschichte, S. 3 f.,
dieser auch für das folgende. eigener Versuche cine "Beschreibung und Abbildung mehrerer Dampfapparate" verfaß $\mathrm{t}^{12}$.

Dingler war also I8I9/2O in fachliterarischen Kreisen, besonders im Bereiche der Färberei und Zeugdruckerei, durchaus kein Unbekannter. Er hatte aber bisher seine publikation vor mich verlegt bei Johann Lconhard chrag in Nürnberg und vor allem in der von Jenisch und Stage'schen Buchhandlung, Augsburg und Leipzig. Wenn man der Vollstïndigkeit der brieflichen Uberliefe rung des Cotta-Archivs trauen darf, hatte er bisher einzis wegen der Licferung einer Maschine geschäftlich un wegen Abbildungen und einer Beilage für das "Morgenblatt für die gebildeten Stände« nur am Rande literarisch mit Cottn korrespondict ${ }^{13}$

Offensichtlich im Bewußtsein der Inangriffnahme eines Offensichtlich im Bewußtsein der Inangriffnahme eines neuen, langfristig angelegten und umfassenden literarischen Projekts hat Dingler, ehe noch der letzte Band se nes "Magazins für die Druck-, Färbe- und Bleichkunst erschienen war, unter Abwendung von seincm bisherigen Verleger, der das Magazin der Färbckunst wegen des guten Absatzes gern fortgeführt hätte, zuerst Cotta gegenüber seinen Plan zur Gründung des Polytechnische Journals entwickeit.

Die Hinwendung zu Cotta lag für Dingler buchstäblich nahe. Seit die »Allgemeine Zeitung«, die wegen ihre einzigartigen auswärtigen Berichterstattung und wegen ihres Feuilletons binnen kurzem zum führenden Blat Deutschlands aufstieg, durch das herzogliche württembergische Verbot getroffen, über die Zwischenstatio Ulm I8xo in das Montgelas-aufgeklärte, weniger autokratische Bayern nach Augsburg ausgewichen war, besa der Cotta-Verlag hier eine Niederlassung und eine Druls ereit4 Gorade aber am Druck des Journals, möglich

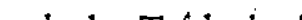
persönlichen Aufsicht und der Möglichkeit zu kurzfristigen Änderungen, Berichtigungen und Einschüben, war Dingler angesichts der monatlichen Erscheinungsweise derart viel gelegen, $\mathrm{da} ß$ er Cotta androhte, falls es »bei der getroffe nen Ubereinkunft, daß dasselbe hier gedruckt werde " nicht bleibe, cr es vorziehen würde, den Titel Polytechnisches Journal seinem schon bestehenden Magazin zu geben. Genauso müßBt der Versand des Journals von

${ }_{12}$ Ưber Dinglers Veröffentlichungen siche Emil Dingler: Nekrolog auf Johann Gottfried Dingler. Polyt. Journal I3 $^{2}$ (I855), S. 396-400; Karl Karmarsch: Johann Gottrried Dingle Allgemeine Deutsche Biographic 5 ( 1877 ), S. 239ff. ${ }^{13} \mathrm{CA} I / x$.2.3 8.2.18x7, ×8.2.1889, I9.3.18x9. Cohrer: Cotta, S. 80.,; Hirbert Scliter: J ohann S. 9 .
Augsburg aus erfolgen, da ssich wohl schwerlich außer Augsburg eine Stadt finden" worde, "die jede Woche durch Bothen und Fuhrleute nach allen Richtungen der Welt Versendungen machen kann « ${ }^{15}$.

Diesen und anderen Einzelforderungen wie überhaupt der gesamten Ubernahme des Polytechnischen Journals in seinen Varlag scheint Johann Friedrich von Cotta in eschäftige, auf Wirksamkeit und Einflußnahme angelege Natur, seine Offenheit gegenüber der technischen und wissenschaftlichen Entwicklung prädestinierten ihn ja geradezu zum Verlag von Zeitschriften und Zeitungen. Die Faszination von Namen wie Goethe, Schiller, Herder u.a. triibt auch nur zu leicht den Blick dafür, daß Johann Friedrich von Cotta allein rund $4 \circ \mathrm{Zcitschriften} \mathrm{verlegt}$ hat und an der Gesantproduktion dieses "Klassiker-

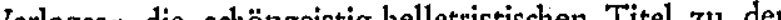
Titel zu den issens Verhältnis von etwa $I$ : Io vertreten waren ${ }^{10}$.

Ein förmlicher Vertrag zwischen Verleger und Herausgeber ist wohl nicht abgeschlossen worden, auch wenn später der Solnn des Gründers, Emil Dingler, meinte, es existiere ein solcher ${ }^{17}$. Herausgeberverträge waren unter Johann Fricdrich von Cotta selbst mit den "Klassikern kum ülich und haben sich bei Cottn erst ab 1835 unte stïker weinaltenden Sohnes Geor Cotta vermehrt ${ }^{18}$.

Nur ein gutes halbes Jahr verstrich zwischen dem Gründungsschrciben und dem Erscheinungsbeginn des Journals. Der Termin Ende Dezember I8xg ließ sich zwar nicht einhalten, doch erschien wohl gegen Ende Jänner I820 das erste Heft des Polytechnischen Journals ${ }^{10}$

Insgesamt waren die Startbedingungen für das Polytechnische Journal nicht ungünstig: Ein naturwissenschaftlich gebildeter und technisch erfahrener Praktike mit erheblicher und erfolgrcicher publizistischer Vergangenheit fungierte als Herausgeber. Dieser stand nach eigenem Bekunden wie kaum ein anderer in ausgedehnten auswärtigen Verbindungen und Korrespondenzen,

S. 4. der Stutef Eberle: Johann Friedrich Cotta. Sonderdruck ats der Stuttgarter Zeitung

CA 112726.x.185s. 10 Liselocte Lohrer (Bcarb.): Schiller-Nationalmuscum Marbach/Neckar. Bestandsverzeichnis des Cotta-Archivs (Stiftun 1. he

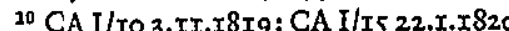


cin Umstand, der für die Nachrichtenbeschaffung von größter Bedeutung sein mußte ${ }^{20}$. Verleger des Journals war aber der Inhaber des bedeutendsten deutschen Verlages, dessen Name allein die Gründung schon bis zu einem gewissen Grade empfahl und dessen buchhändle tische Verbindungen, Fin rische Vers de $\mathrm{G}$. zige« Begeisterungseahigkeit fur die Emporbringung de "vaterländischen lndustie" hoffen ließen, die bei Zeitschriftengründungen erfahrungsgemäß schwierigen Anfangsjahre zu überwinden ${ }^{21}$

Herausgeber und Verleger waren auch Männer mit einem bei Dingler erheblichen, bei Cotta aber sehr großen Ansehen und Einfluß bis zu den höchsten Kreisen, speziell in Bayern und Württemberg, aber auch in Preu

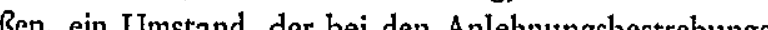
an an die am Steigerung des Absatzes nicht außer acht $z \mathfrak{z u}$ lassen ist. Sieht man von den Gewerbevereinsblättern ab, war das Polytechnische Journal auch die erste private Gründung dieser Art ohne anfängliche Konkurrenten.

Besonders im Hinblick auf den Absatz schcint zunächst entgegen der Ansicht Dinglers der problematischste Punkt der Gründung die an sich gute Verkehrsh des Druck- und Versandortes Augsburg gewesen zu scin. Aoring las fir sudd vorteilhat und war in gunstiger Escheinungsort fü cine Zeitung, welche wegen ihrer zunehmend großdeutsch-liberalen politischen Richtung in ihrem Absatz ohnehin mehr in den süddeutsch-österreichischen Raum tendierte22. Für ein Journal, das angesichts eines begrenzten, überhaupt ansprechbaren Leserkreises seinen Absatz nach Möglichkeit im gesamten Deutschen Bund anstre ben mußte, lag Augsburg aber eher unginstion Versandkosten mit der Enferte en und solunge sen und solngesich keiten nicht besserten, sind daher die gelegentlichen Klagen über den unterproportionalen Absatz des Journals in Norddeutschland ${ }^{23}$ verständlich, also in das Gebiet in welchem neben Westdeutschland in der Folgezeit die

${ }^{20} \mathrm{CAI} / 6$ I4.8.I8I9.

${ }^{21}$ Ähnlich wie schon im Vorwort zum I. Band des Polytechnischen Journals, S. VI, schreibt Dingler aucl CA Pol/y 5.4.r826, daß das Polyt. Journal svon seiten der Verlagshandlung mehr der Vaterländischen Industric als $\mathrm{dcm}$ eigene Interesse wegen gegründet wurde.« Desgleichen Polyt. Journa 33 (1829), S. 477

${ }^{21} \mathrm{CA} I / 37$ I5.rI.x82x schrieb Dingler an Cotta: "Da dic allgemeine Zeitung und das Morgenblatt in dem nördl. Deutschland wenig und nur von Statatsdienern, nicht aber vo ${ }^{23}$ CA I/89 8.xx.1825; CA II/99 undaticrt (I846).
Industrialisierung rascher und zügiger voranschritt als in den wirtschaftlich relativ zurückfallenden alten Gewerbelandschaften Süddeutschlands.

\subsection{Die Zielsetzung}

Wie im allgemeinen üblich, gibt der Titel der Zeitschrift bereits einen entscheidenden Hinweis auf deren Programm. Allerdings ist der damals als modern, fortschrittlich, ja geradezu als revolutionär gef̈̈lolich empfundon Beriff polytech zuschillernd, zu und $z u$ schr der Wandlung unterworfen gewesen, als daß man einzig anhand des Titels bereits konkret die Zielsetzung des Journals umreißen könnte. Dingler hat in seinem Gründungsschreiben an Cotta und im Vorwort des Journals selbst niedergelegt, was er unter Polytechnik verstand und wie er der Tendenz der Zeitschrift nachkommen wollte. Er wollte in seiner Zeitschrift whs game Gebiet der Polytehnike abdecken, palso, die allgemeinc issenschaft, die Chemic, die Mineralogic, die Pflanzenkunde, die Land- und Hauswirtschaft, dic Maschinenlehre und Gewerbskunde, die Handels- und Warenkunde « ${ }^{25}$. Wie er an Cotta schrieb, wäre die "Tendenz « des Journals »alle gemeinnützige, inn- und ausländische, auf Erfahrung gegründete Erfindungen pp. in Künsten, Manufakturen, Fabriken, Ge werben $\mathrm{Pp}$ in einem allen Ständen foßlichen Vortabe zu

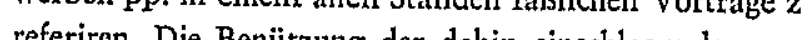
lischen Die Bench lischen, französischen und italienischen Literatur so wie meine und meiner zahlireichen Freunde viclseitige eigenthümliche Arbeiten würden mich in Stand setzen ... eine Zcitschrift zu gründen, die, fern von aller Ruhmredigkeit, bis jetzt noch kcine Nazion aufzuweisen hat «11.

Das Polytechnische Journal solle "allgemeine Brauchbarkeit mit Gründlichkeit und Vollstت̈ndikeit so vich möglich vercinisenc und er hofe nau disse Art durch Sammlung rischen rischen und der praktischen Betriebsamkeit in dem polytechnischen Gebiete dic Idee eines dem Landmanne, wic dem Fabrikanten, dem Handelsmanne wie dem Handworker, dem Künstler wie jedem wissenschaftlich gebildeten Manne gleich angenehmen und nüzlichen Repertoriums $\mathrm{zu}$ verwirklichen $\star^{25}$.

Mit seiner weiten Inhaltsbeschreibung der Polytechnik erweist sich der $x 778$ geborene und noch im 18 . Jahrhundert erzogene Dingler als ein Mann des Übergangs.

${ }^{24}$ Troitzsch: Entwicklung, S. 333; Treuc: Dinglers Poly-

technisches Journal, S. 6 f., Ir beide auch für das folgende.
Das technische Stoffgebiet hat sich bei ihm noch keineswegs aus der Verklammerung mit anderen Wissensbereichen befreit. Besonders die Berücksichtigung der Maschinenlehre und die Einbezichung der allerdings frühe bereits gut in der Zeitschriftenliteratur repräsentierten in ung positiv vo den kameralistisch-tcchnologischen Zeitschriftenvorläucrn ab. Mit der Handelsn und Warenkunde, der Pflanzenkunde und der Land- und Hauswirtschaft beabsichtigte er allerdings - soweit nicht deren technische und naturwissenschaftliche Grundlagen gemeint waren ein merkantiles und agronomisches Gebiet einzubezichen, das selbst unter dem polytechnischen Aspekt zunehmend nicht mehr in Beziehung zur technischen Be Inhalt der Gewerbekunde exakt zu fassen, da cinerseits der Begriff Gewerbe einem ökonomischen Ordnungsprinzip gehorcht, andererseits das Produktionsverfahren eine handwerkliche oder technische Leistung darstellt ${ }^{20}$ Es scheint aber, daß diescr im Vorwort des Journal angesprochene weite Themenkreis auch im Hinblick au die Werbung und dic Gewinnung von Abnchmern formuliert worden ist. Im Gründungsschreiben an Cottn ist Jeden schrift mit der Referierung aller auf Erfahrung gegründeten Erfindungen in Künsten, Manufakturen, Fabriken und Gewerben sehr viel technikbezogener umschrieben.

Es ist noch zu fragen, wie sich die Zielsetzung des Polytechnischen Journals von den glcichzeitig aufkommenden Gewerbeblättern abhob und wodurch denn das Journal seine Eigenart und innere Berechtigung erhielt. Beabsichtigte doch Dingler, ähnlich wie die Gewerbeveringlite doch Dinglen the Versuche und Prïfungen empfohlener Gegenstände zu veranstalten.

Als private Gründung besaß das Journal keinen Rückhalt an einem organisierten Leserkreis. Es entfielen daho bei ihm die für den Zusammenhalt der Mitglieder wichtigen Vereinsnachrichten. Die gewerblichen Zeitschriften berücksichtigten zur Unterrichtung und Bildung der $\mathrm{Ge}-$ werbetrcibenden besonders in den Anfangsjahrzehnten ach vich otirker wirtshefliche und handelskundliche liche Fragen, wahhrend der technische Nachrichtendien "offensichtlich die schwichste Seite der Gewerbeverein blätter" war ${ }^{27}$. Gerade in der von Vereinsrücksichtsnahmen unabhängigen Stoffauswahl, der raschen Nachrichtenvermittlung und in der Vorrangigkeit des technisch-

\section{${ }^{20}$ Hamann: Zeitschriften, S. 100}

${ }^{27}$ Schulz: Fachpresse, S. 35 . naturwissenschaftlichen Inhalts lag aber - wie im einzelnen anhand der Inhaltsanalyse noch zu zeigen sein wird - der Schwerpunkt des Polytechnischen Journals. Das Polytechnische Journal hat den Typ der mehr technisch ausgerichteten, meist privat initiierten polytechnischen Zeitschriften bcgrïndet und geprägt. Da sich allerding a ch der Gewerbevercinsliteratur mit der Technik befaßten und da in der wissenschaftlichen Diskussion unterschiedliche Auffassungen vertreten werden über die Zuordnung der gewerblichen Blätter $\mathrm{zu}$ den wirtschaftlichen oder den technischen Zcitschriftenn ${ }^{28}$, wird man die wertende Frage offenlassen, ob das Polytechnische Journal die crste technische Zeitschrift Deutschlands war.

Das Polytechnische Journal wird in der Literatur auch wiederholt als wissenschaftliche Zeitschrift bezeichnet ${ }^{20}$. Zunächst fällt es schwer, die Bezcichnung "wissenschaftlich" für eine Zeit zu gebrauchen, in der die Technik noch um ihre wissenschaftlichen Grundlagen rang und in der Erfindungen anstatt auf methodisch-experimentellem oder nachpriffbar spekulativem Wege noch durchaus aufgrund des fachlichen Geschicks, der Erfahrung und einer gewissen praktischen Experimentier- und Probierfreudigkeit gemacht wurden Man findet nun in der Tat Poly Polch ichen Chater tragen. Die Fortshritte der Wissenschaften sollten sich aber in dem Polytechnischen Journal nur insoweit spiegeln, als diese dem Gewerbetreibenden "helfend und rathend an die Hand" gingen und diesen in den Stand setzten, waus sich selbst eine neue Schöpfung hervorgehen zu' lassen $\mathrm{\aleph}^{30}$. Nach seiner Zielsetzung und tatsächlich war damit das Polytechnische Journa keine originer wissenschaftliche Zeitschrift. Bs war vielmehr ein Referateorgan, welches zwar auch Originalbeiträge aufnahm, das aber in erster Linie aus bereits veröffentlichter Literntur schöpfte und das dic technischen Neuerungen, Erkenntnisse und Forschungen in verständlicher Form den weiteren Kreisen der Polytechniker vermitteln wollte. Dingler selbst lchnte zum Beispiel ז82x die Ưbernahme eines Artikels mit der Begründung $a b$, daß der Gegenstand win wissenschaftlicher Hinsichtu alle Beobachtung verdiene, er eirne usich daher

${ }^{20}$ Hamann: Zeitschriften, S. I00 Anm. 2 und S. xosf. ${ }^{20}$ Troitzsch: Entwicklung, S. 332; Helmuth Dingler: Johan Gottfried Dingler, Neuc Deutsche Biographic 3 (x957), S.730 ${ }^{30}$ Polytechnisches Journal 4 (x82x), S. 488. Diese Formulierungen sind von Dingler gcbraucht worden für die Begründung der Notwendigkeit der Errichtung von Gewerbevereinen, 


\subsection{Die Herausgeber}

Die beträchtliche redaktionelle Arbeit wurde beim Polytechnischen Journal lange Jahre hindurch von einem einzigen Mann geleistet, der überdies als Fabrikant noch einem Hauptberuf nachging. Der Begründer des Journals, Johann Gottfried Dingler, firmiert vom Jalre $x 82$. an bis zu seincm Tode im Jahre 1855 als Herausgeber auf dem Titclblatt. $\mathrm{T} 83 \mathrm{r}(\mathrm{Bd}$ d Dingler hinzu. $\mathrm{Ab}$ 834 (Bd. 5I) wird Emil Dingler neben Julius Hermann Schultes bis zum Jahre 1840 (Bd 77) als Mitredakteur aufgeführt. 1840 bis 1855 erschien das Blatt wiedcrum unter der Herausgeberschaft von Vater und Sohn Dingler. Nach 1855 zeichnete bis 1874 Emil Dingler allein als Herausgeber.

Die Lebenswege dieser drei Personen sind aus ihren Die Lebenswege dies iographien in der Allgemeinen Deutschen Biographie und in der Neuen Deutschen Biographie hinreichend bekannt. Hier sollen daher nur dicjenigen Stationen ihres Werdegang und ihrer Berufstätigkeit nachgezeichnet werden, welche für die Redaktion des Polytechnischen Journals von Wichtigkeit geworden sind.

Aus den Briefen des Cotta-Archivs ergeben sich überdies einige neue, über die bisher bekannten Fakten de Biographien hinausfihrende Tcilaspekto, auf die hier wegen ihrer Bedeutung exkursartig in Kürze eingegange wcrden soll. Der eine Aspekt beleuchtet einige zunächs wohl aus Pietät im Nekrolog etwas zu kurz geratene später aber vergcssene Umstände der unternehmerische Existenz Johann Gottfried Dinglers. Der andere Aspek betrifft das Verhältnis Cotta-Dingler, also von Verlege und Herausgeber, in ihrem nicht die Probleme der Redaktion des Polytechnischen Journals unmittelbar be treffenden Teil. Er vermag einiges beizutragen zum Ge schäftsstil und zum Umgang der. Inhaber des Veros hauses Cottn mit den Autoren sowio auch bestes Johann Gottfried Dingler und kann daher auf allgemeineres Interesse rechnen.

\subsection{Johann Gottfried Dingle}

Als Sohn eines Leinenwebermeisters am 2. I. I778 in Zweibrïcken geboren, wurde Johann Gottfried Dingle

3x $\mathrm{CAI} / 35$ 27.8.x82x. durch Vermittlung eines Hofbeamten Apotheker. Nach einer Stelle als preußischer Feldapotheker ließ er sich im Jahre 1800 als selbständiger Apotheker in Augsburg nieder. Hier in Augsbure dem damaligen Zentrum der nieder. Hin in süddeutschen Kattunmanufaktur, veranlaßste ilhn die Bekanntschaft mit dem "deutschen Kattunkönig", dem Augsburger Fabrikanten J. H. von Schüle, sich als ausübender Chemiker der Druck- und Färbckunst zu widmen. Nach dem Studium der Zeugdruckerei im elsässischen Mülhausen, wohin er auch r809/xo zur Unterrichtung in der. Türkischrotfärberei ging, gründete er I806 in Augsburg unter der Firma "Dingler und Arnold" eine Fabrik chemischer Produkt dic er spater auf allenige Rechnung weiterführte. Im Jahre des Beginns seiner Fabrikantenlaufbahn und seiner publizistischen Tätigkeit, also I806, erhielt er von der philosophischen Fakultät der Universität Gießen den Doktorgrad verliehen. x822 erwarb er noch eine sistierte Augsburger Kattundruckerei ${ }^{32}$.

Abgesehen von der für die Redaktion des Journals günstigen naturwissenschaftlich-technischen Konstellation in Ausbildung und Beruf Dinglers ist an diesem Lebenslauf bemerkenswert, daß das Polytechnische Journal damit von einem Mann gegründet worden ist, der durch seine Ausbildung und seinen Beruf denjenigen zwei Schlüsselbereichen der industriellen Revolution verbunden gewesen ist, in denen sehr frühzeitig Maschinen eingesetzt und die periodische Berichterstattung zur Vermittlung neuer Erkenntnisse herangezogen wurden. War doch die Textilindustrie in der Frïhindustialisierung

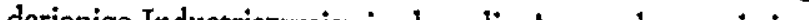
derjents sparender Maschinen am weitesten fortgeschritten war. Und auf dem Gebiet der Pharmazie und Chemic waren neben der Montanistik infolge des Interesses des aufgeklärten Publikums an den Naturvorgängen und Naturwissenschaften sowie infolge der im $x 8$. Jahrhundert einsetzenden Konsolidierung der Chemic als einer rationalen Disziplin schon seit den $1770 e r$ und 1780 er Jhren eine Reihe von Zeitschriften entstanden ${ }^{33}$

Es scheint, daß Dingler mit der $x 822$ übernommenen Kattunfabrik, die er noch durch Neubauten erweiterte, sich finanziell übernommen hat. Er geriet zu dieser Zeit wegen der Konzessionierung einer von ihm geplanten Schnellbleiche und der Genehmigung einer Schwefel-

${ }^{\text {sa }}$ Siehe oben Anm. I2 und 29 sowie Wolfgang Zorn: Augsburg. Geschichte einer deutschen Stadt (Augsburs Igssh, S. $230 ; \mathrm{CAl} / 44$ a 14.5 .1822

Schulz: Fachpresse, S. 23 ff.; Albrecht Timm: Einführung in dic Wissenschaftsgeschichte (Uni-Taschenbücher 203, Mün-

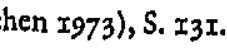

säurefabrik auch in langwierige und kostspielige Auseinandersctzungen in der Stadt Augsburg ${ }^{34}$. Jedenfalls ceißen in den Bricfen an Cotth nunmehr die Khagen über die Ebbe in den Kassen, den Mangel an Finanzleredi und an liquiden Mitteln nicht mehr ab, denen beinahe stets Bitten um Darlehen und Vorschußzallungen auf das Herausgeberhonorar folgten. Die finanziell nunversiegbare Handlung" Cottas kam diesen Hilferufen offensichtlich großzügig nach. Sie übersandte manchmal mehr, als gefordert war. Dingler wußte »dieses Zuvorkommen zu schäzen« und fühlte sich durch diese dauernde Unterstützung zum doppelten Einsatz seiner Kräfte für das Polytechnische Journal verpflichtet ${ }^{35}$.

Die gewaltig differierenden Angaben über die Höhen der Geldsummen, dic er zur Emporbringung der Fabrik $\mathrm{zu}$ benötigen glaubte, werfen nicht das beste Licht auf Dinglers wirtschaftliches Augenmaß. So richtete er $x 826$ an den bayerischen König ein Gesuch um ein Darlehen von nicht weniger als 50000 bis 60000 Gulden zur Anschaffung von Maschinen. Nur ein halbes Jahr später meinte er, offensichtlich mit einem Seitenblick auf eine eventuelle Beteiligung Cottns, wenn ihm unur cin Freund in etlich mit etllichen ause ann könnte dicse Indus (die Kattunfabrikation, $d$ Verf.) auf die Basis einiger neuer Erfindungen bald blühend und lohnend werden $«^{30}$.

Das Spielen mit der Drohung des Zusperrens der Fabrik, die etwas sanguinischen Hoffnungen, die sich an vage Andeutungen von möglichen Kreditzusagen und den Einsatz neuer, allerdings wegen Kapitalmangel aucenblicklich nicht zu realisierender neuer Erfindungen M Maschen Briefen zu Projekte, welche bis zur Errichtung einer vom König zu privilegierenden Schwefelsäurcfabrik in Württemberg - natürlich im großen bei völliger eigener Finanzblöße - reichten, lassen seiner unternehmerischen Erscheinung etwas vom vielgeschäftigen Projektemacher des $\mathrm{I} 8$. Jahrhundert anhaften ${ }^{37}$. Auch hier ein Mann des Ubergangs und getrieben yon der latenten Finanzklemme, die ihn noch jedem Strohham Ausochau halten ließ lag sciner mach Bildungswer und Interessen breit angelegten Natur wohI

34 Alexander Allwang: Augsburger Straßennamen - Dingler(raße. Alt-Augsburg, Jg. x, H. 4/5 (I937), S. 77f, Anm. x6; $\mathrm{CAI} / 703 \mathrm{Y} . \mathrm{I2.I823}$

${ }^{36} \mathrm{CA} I / \mathrm{I}_{35} 2 \mathrm{2T} .5$.(3) $x_{227}$; CA I/66 4.xx.x823; CA I/83 3.3. I825.

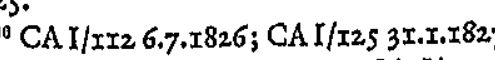

${ }^{37}$ Z.B. CA I/IO2 II.3.I826; CA I/II2 6.7.r826; CA I/XI8 9.8.5826. auch kaum der Typ des neuen industriellen Unternehmers des Ig. Jahrhunderts, der alle seine Kräfte auf sein wurde und der kaum $Z$ in fand fïr

Dingler suchte vielmehr, über seine Fabrikanten- und publizistische Tätigkeit hinausgehend, ngemeinnützige Betätigung", und sein »exprobter Patriotismus « und "bekannter Eifer für das allgemeine Beste « fanden auch öffentliche Anerkennung. So wurden seine "ausgebreiteten Kenntnisse in Allem, was auf Handel und Industrie Bezug hat holt von den Behörden in Anspruch genommen. 1826 wurde er $z \mathrm{~B}$. mach einem "Kraftschreiben an den bayerischen König wesen der Übergehung der Fabrikanten an den Zollberatungen selbst in diese Kommission berufen, wo er gleich zum Referenten gewählt wurde ${ }^{99}$.

Ähnlich wie beim Baron von Cotta, mit dem sich hie eine äußere Parallelität exgibt, ist Dinglers unternehmerisches, polytechnisches und gemeinnütziges Wirken unter den deutschen Staaten am meisten von Bayern, Württemberg und Preußen anerkannt und durch Auszeichnungen honoriert worden. So übersandte ihm beispiclsweise der proußische Ministar von Schuckmant als Anwise der prish verlich ih en pach Ministerium sandte ihm aus gegebenem Anlaß ein Ehrenschreiben $^{30}$.

Als politischer Mensch hielt er mit seiner Meinung nicht hinter dem Berg, und in seinen Briefen an Cott weist er wiederholt auf seine öffentliche Wirksamkeit Einflußmöglichkeit und seine persönliche Bekanntschaft mit hohen Beamten und Ministern hin. Diese Aktivität beschränkte sich nicht auf die als Ausfluß seines beruflichen Wirkens im weiteren Sinne zu betrachtende Mitarbeit an Ministerkommissionen oder Mitgliedschaften und Ehrenmitgliedschaften in den verschiedenen naturforschenden, gewerbefördernden, polytechnischen udgl Gesellschaften, welche er auf dem Titelblatt des Polytechnischen Journals durch Jahrzehnte fein säuberlich aufzählt. Er besaß vielmehr auch das Vertrauen vieler sciner Mitbürger.

I828 wurde Dingler bei der durch die Kreisveränderung notwendig gewordenen neuen Wahl der Landräte als Kandidar aus der Khsse der Städte und Märkte fïr den Kreis Schwaben und Neuburg gewählt und vom

CA I/I2I x4.8.I826; CA I/I21a 4.8.I826; CA I/250a 2.9. .5844

${ }^{30} \mathrm{CA} \mathrm{I} / \times 23$ I6.I.x827; CA I/233 a x6.I.x835; Polyt. Journal $7(1822), S .254$. 
König Ludwig I. wider sein Erwarten als Landrat ernannt. Dingler war auch Mitglied des Kollegiums der Gemeinde-Bevollm Wunsch der Bürge versammlung gewählt werden, was aber nach Dingle Angaben durch Umtriebe sinistrer Gegner verhindert wurde ${ }^{40}$.

Wesentlich ungünstiger als seine öffentliche Stellung entwickelte sich jedoch die wirtschaftliche Lage seiner Firma. Und dies $z \mathfrak{u}$ einer Zeit, als durch die Errichtung des Zollvereins zum Jahresbeginn I834 für das Augsburger Wirtschaftsleben, besonders auch die Texrilindustric, nach jahrzehntclanger Bedrängnis ein neuer Aufstric, nach jahrzehn
schwung einsetzte ${ }^{11}$.

Die Knappheit an langfristigem Anlagekapital und laufendem Betriebskapital, die neben Darlehen von mehreren tausend Gulden manchmal auch Vorauszahlungen von wenigen hundert Gulden an Dingler durch Cotta für Badeaufenthalte oder Reisen zu Industrieausstellungen willkommen sein ließen, hatten schon $x 830$ eine gerichtlice Taxation einiger Fabrikgrudstüce Dingles ur Fongers zur Folge gether Besserung der wirtschaftlichen Lage Anfang der $x 830 e r$ Jahre wurde diese im zweiten Jahrfünft der 1830 oc Jahre nach den Briefen an Cotta zu schließen wieder prekärcr. Am 30. November $x 843$ teilte er Cotta mit, »durch ein unvorhergesehenes Zusammentreffen von Finanzverhältnissen und deren Folgen war unsere Firma genöthigt die Zahlungen einstweilen zu suspendieren “, Nach einem gedruckten Circular Dinglers man die vorchliche Gesammt-Creditorschaft von Dingler \& Comp. in Augsburg" standen einem höchstens $98000 \mathrm{Fl}$. « Passiva von circa $253000 \mathrm{fl}$. gegenüber ${ }^{43}$.

Der Besserwisscr war, zumindest wirtschaftlich, kein Besserkönner gewesen. Seine persönliche Tragik war, daß scine Firma fallierte, als er schon im hohen Alter stand, und daß weitere von ihm initiierte Sanierungsversuche, unter anderem ein Plan zur Gründung einer Aktiengesellschaft, scheiterten.

Das ungebrochene Verhältnis Johamn Gottfried Dinglers zum Schuldenmachen und Ubberzichen seines Honoraranteils veranlaßte 185 I seinen Mitherausgeber und Sohn Emil Dingler, seinem 74jährigen Vater und dem

\section{${ }^{30} \mathrm{CAI} / \mathrm{x} 56$ xx.2.1828; CA I/20x Ix.I.x83x.}

"Zorn: Augsburg, S. 247f.

${ }^{42} \mathrm{Z} . \mathrm{B} . \mathrm{CA} \mathrm{I} / \mathrm{IO2} \mathrm{Ir} .3 .1826 ; \mathrm{CAI} / 24 \mathrm{I} 2.6 .1837 ; \mathrm{CA} / 243$

2x.5. 1838 .

${ }^{13} \mathrm{CA}$ I/249 30.1I.r843; CA I/250a 2.9.1844; CA I/256

I9.4. $\times 846$.

Verlag Cotta gegenüber den Rücktritt von der Redaktion anzudrohen für den Fall, daß der Verlag das Schuldenmachen des Vaters auf Kosten des Honoraranteil des Mitherausgebers weiter unterstütze ${ }^{44}$.

Am 19. Mai 1855 ist Johann Gottfried Dingler in Augsburg verstorben.

Angesichts der Doppelbelastung von Vater und Sohn Dingler durch die Leitung der Firma und die Redaktion des Polytechnischen Journals, und da eine ungefähre zeitliche Koinzidenz festzustellen ist zwischen dem $\mathrm{Be}$ ginn der wirtschaftlichen Misere und der Gründung des Journals, ist zu fragen, ob etwa die Herausgabe des Journals die Krafte der Firmeninhaber derart absorbie te daß für die Firmenlcitung zu wenig Zeit übrigblieb. Die Redaktion eines praxisbczogenen Referateorgans von der Art des Polytechnischen Journals verlangte von seinen Herausgebern ohne Zweifel dic dauernde intensive Beobachtung des Standes des Gewerbclebens sowie der Fortschritte der Technik und der Naturwissenschaften in der Praxis und in der Literatur in allen ihren Zweigen Das Iesen Sichten und Auswällen der Stoftmassen in der Zeitschriftenliteratur, die Beschaffung der Zeitschriften sclbst, das Anfertigen von Auszügen und Redaktionsnotizen, die Korrespondenzen mit den Mitarbeitern und dic Uberwachung der Korrektheit von Artikelinhalt, Druck und Zeichnungen exforderten von den Herausgebern neben umfassenden Sachkenntnissen und einem gewissen organisatorischen Talent allein schon so viel mechanische Tätigkeit, daß man sich wundert wie Deit von den beiden Dingler oder einem von allein durchgeführt werden konnte. Dies um so mehr, wenn man sich vor Augen hält, daß je ein Heft des Journals pünktlich zuerst monatlich, später alle $I_{4}$ Tage der Offentlichkeit vorgelegt werden mußtc. Ob der Buchhalter oder der Korrespondent, die Johann Gottfried Dingler in seiner Firma besa $\Omega^{45}$, für die Abrechnungen oder Korrespondenzen zum Polytechnischen Journal herangezogen wurden, wissen wir nicht.

Trotz der sicherlich hohen Arbeitsbelastung durch die Redaktion, darf man bei der Frage der Verträglichkeit von Firmen- und Redaktionsbeanspruchung nicht außer acht lassen, daß Johann Gottfried Dingler bereits in früheren Zeiten redaktionelle und schriftstellerische Arbeiten durchgeführt hat, ohne daß offensichtlich die Firma Schaden genommen hatte. Auch ist es mit der Firma crst zu einer Zeit sichtlich bergab gegangen als Vater und Sohn gemeinsam Unternehmer und Herausgeber

${ }^{44} \mathrm{CAII} / 272$ 26.r.r85 r.

${ }^{46} \mathrm{CA} I / 98$ 19.5.5826. waren, also als die Möglichkeit zur gegenseitigen Entlastung und Arbeitsteilung am günstigsten war. Man wird daher den Anteil der Beanspruchung der Firmeninhaber durch die Herausgabe des Journals am Untergang der Firma nicht überbewerten dürfen.

\subsubsection{Emil Maximilian Dingler}

Am Ir. Dezember 1830 teilte Johnnn Gottfried Dingler dem Verlag Cotta mit, daß sein Sohn Emil Maximilian Dingler am $\mathrm{I}$. November Teilhaber an Fabrik und Handelsgeschäft geworden sei, und or ab Jänner I83r yals Mitredacteur od polytechnischen Journals auf dem Titel aufgeführt" polytech.

Dieser war am xo. März I806 in Augsburg geboren worden und führte als Taufnamen eigentlich den Vornamen "Napoleon«. Allein schon in der Wahl des später wieder ominös gewordenen und verdrängten Namens lag eine politische Demonstration von seiten des Vaters! Dinglers "braver Emil« hat das Gymnasium seiner Vaterstadt mit außerordentlichen niversität andshut, bei dem berühmten Trommsdorff in Erfurt, dann in Berlin und Göttingen. I829 promovierte er in Erlangen zum Dr. phil. Ehe er in Firma und Mitredaktion cintrat, hat er eine längere, nach Ablehnung eines Reisestipendiums durch das bayerische Ministerium weitgehend durch Vorschüsse vom Verlagshaus Cotta finanierte polytechnische Studienreise absolviert, durch

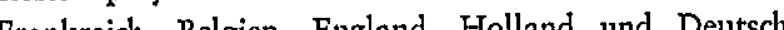
Holland und Deutschland 47

Emil Dinglers gediegenen naturwissenschaftlichen Studien, die auf der Reise gewonnene Auslandserfahrung, sein eigentlich erst dabei geweckter "Sinn fürs Gewerbsleben und Industrie«, ein außerordentliches Gedächtnis und sein Fleiß, die ihn schon im Gymnasium zwei Klasen hatten überspringen lassen, und eine »ihm angeborene Grïndlichleit đ8 befähigten ihn ausgezeichnet für die Redalion des Joumals chere Natur als sein Vater. Vor allem ging ihm offensichtlich dessen Streben nach öffentlicher und politische Wirksamkeit ab. Er konzentrierte vielmehr alle seine Kräfte auf die Herausgabe des Journals. In seinem $\mathrm{Ne}$ krolog rühmt Karmarsch wiederholt seine Bescheiden-

10 CA I/x99 Ix.x2.1830.

${ }^{47}$ Karl Karmarsch: Nekrolog auf Emil Maximilian Dingler, Polyt. Journal 2I4 (I874), S. I-VII; CA I/776 22.10.x829 CA

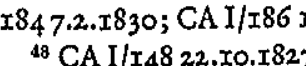

heit. Die Bemerkung von demselben Karmarsch im Nekrolog, daß Emil Dingler die Redaktion des Journal "vom 78. Bande $(x 840)$ an in seine alleinige Hand nahm «, ist wohl dahingehend zu verstehen, daß $\mathrm{ihm}$ zunchmend die Hauptarbeitslast der Redaktion zufiel. Es scheint damals auch eine fachbezogene Arbeitsteilung wischen ihm und seinem Vater durcheführt worden zu in we auf Emil Dinger mit der Beabeiung der wing der mechanischen und chemischen Technologie, für welch er durch sein Studium auch am besten vorbereitet war, der weitaus gewichtigere und zukunftsträchtigere Part
uibergegangen war $^{40}$.

Der Untergang der väterlichen Firma, an welcher er zu einem Drittel Teilhaber an Gewinn und Verlust war, weckten in ihm den Plan zur Bewerbung um eine Professur an einer höheren Gewerbe- oder polytednischen Schule. Dem polytechnischen Journal sollte dabei eine idoppelte Aufmerksamkeit gewidmet《 werden ${ }^{\text {to }}$. Ohne daß dicser Plan realisiert wurde - vielleicht waren auch alle Bemühungen zu scincr Realisierung Fchlschläge besorgte Emil Dingler bis ein halbes Jahr vor seinem Tode in einer staunenswerten Arbeitsleistung ohne irgendwelchen fachmännischen Beistand die Herausgabe des Journals. Gesundheitsbedingt gab er zum 3x. März 874 dic Rechltion der Zeitschrift auf und willigte noch cin in die Umbenennung des Titels ab April 1874 in "Dinglers Polytechnisches Journal", herausgegeben von Prof. Zeman und Dr. Fischer. Er starb bereits am 9. Oktober $1874^{\text {s1 }}$. Sein großes herausgeberisches Verdienst war es, die Zeitschrift unter allmählicher weiterer Steigerung des Absatzes erfolgreich aus der frühindustriellpolytechnischen in die industrialisiertere und "fachwissenschnftlichere« Zeit der Reichsgründungä̈rn hineingefühtt zu haben.

\subsubsection{Der Mitredakteur}

Julius Hermann Schultes

Für sechs Jahre steht als Mitredakteur in den Jahren r834-r840 Dr. Julius Hermann Schultes (I804-I840) auf dem Titelblatt des Polytechnischen Journals. Er war der Sohn des mit Johann Gottfried Dingler eng befreundeten kgl. bayer. Hofrats und Professors, Direktors der chirurgischen Schule in Landshut, Dr. med. Joseph August Schultes (I773-r83r), mit dem ihn vor allem auch der Gleichklang der politischen Ansichten verband. Ding-

${ }^{40} \mathrm{CA} \mathrm{III} / 2726 . \mathrm{x} \cdot \mathrm{I8} 5 \mathrm{x}$

${ }^{80} \mathrm{CAI} / 250 \times 4.9280$

${ }^{21} \mathrm{CA}$ II $/ 94$ I6.x2.I873; CA II/98 r.3.I874. 
ler hat sich für den jungen Schultes wicderholt seit $x 826$ bei Baron von Cotta verwandt, um diesem entweder eine Professur für Naturgeschichte oder Chirurgie einerseits, zww. irgendeine hen Verlagshandlung zu verschaffen ${ }^{52}$.

Julius Hermann Schultes hatte in Wien die Handlun exlernt, hatte gründliche botanische, dann physikalisch chemische und anatomische Studien betrieben und studierte schließlich Medizin. 1825 schloß er mit der Promotion in Landshut $\mathrm{ab}$. Trotz seiner breit angelegten Ausbildung, guter wissenschaftlicher Anlagen und vielA itiger Sprachkenntnisse fand Julius Hermann Schultes offensichtlich auch eine olge der Isolierung, in die sein rigoros aufklärerisch gesonnener Vater in der bayerischen Restaurationszeit nach dem Sturz des Grafen Montgelas im Jahre 18r7 geraten war. 183 I hat sich der junge Schultes in München als praktischer Arzt niedergelassen. Vielleicht mitbedingt durch dic Uberlegung Dinglers, daß diesem dann die Unterstützung seiner fünf unversorgten Geschwister leichter follen könnte, und wohl auch aus Verpflichtung genüber seinem verstorbenen Freund hat Dingler den jungen Schultes I834 als Mitredakteur »engagiertu. Er sollte "mchr wissenschaftliche als technische englische Journale bearbeiten". Neben seiner Arztpraxis und seinen wissenschaftlichen Arbeiten hat Schultes bis zu seinem frühen Tode im Jahre 1840 "täglich mehrere Stunden « für das Journal verwendet, und »er lieferte dafür nicht nur Übersezungen aus englischen, französischen, italienischen und holländischen Zeitschriften sondem

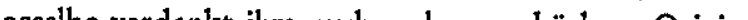
dasselbe verdankt ihm auch mehrere schäzbare Original-

Allen drei Herausgebern bzw. Mitredakteuren in den ersten 50 Jahren des Polytechnischen Journals gemeinsam ist die Verbindung zur Praxis durch ihren stets oder vorübergehend ausgeübten Hauptberuf, ihre breite naturwissenschaftliche Bildung und ihr Streben nach wissenschaftlicher Erkenntnis.

52 CA 1/123 x6.x.x827; CA I/I25 3x.x.x827; CA I/x34 27.4.

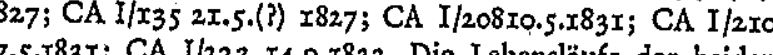
17.5. 583 II; CA $1 / 223$ I4.9.7833. Dic Lebensläufe der beiden
Schultes siche in: Nekrolog auf Joseph August Schultes, Polyc. Journal 42 (I8 3 I) S. 222-232.

Nekrolog auf Julius Hermann Schultes, Polyt. Journal 78 ( 1840$)$

S. 77-80.
${ }^{63} \mathrm{CA} \mathrm{I} / 227$ x8.2.x834; Nekrolog, Polyt. Journal 78 (I840),
S. 80. 8

2.4 Das Verhältnis Verleger -

Herausgeber

Der außerliternrische Rulm Johann Friedrich von Cottas Gü̈ndet darin, daß seine Bedeutung als VerfassungsTont Technik und Unternehmer großen Stils scincr überragenden Stellung als Verleger nicht viel nachsteht. Bei derart vielschichtigem und hochgestimmtem Streben kann es daher nicht wundernehmen, daß sich die Briefe Johann Gottfried Dinglers keineswegs auf die Belange des Polytechnischen Journals allein beschränken, zumal Cotta in Dingler einen ähnlich komplexen Partner, allerdings Jer Aufgreifens der verschiedenen Themen, zusätzlich zu de redaktionellen und 'den Dingler-Firmenproblemen besonders literarisch-verlegerische, wirtschaftlich-technisch und politische, speziell zollpolitische Fragen, angeschnit-

So vermitteltc Dingler wiederholt Autoren aus seinem Bekanntenkreis an Cotta, welche ihre Utbersetzung, ih Aufsatz- oder Buchmanuskript gern in einer Cotta'schen Zeitung oder Zeitschrift abgedruckt oder von Cott verlegt gesehen hätten $n^{54}$. Er gab selbst Anregungen zu Aufnahme von Titeln in das Cotta'sche Verlagsprogramm, gelegentlich riet er auch von der Ubernahm eines Werkes in den Verlag $a b^{65}$. Dingler fragte auch wiederholt an, ob er aus den für das Polytechnische Journal gehaltenen ausländischen Zeitschriften UbersenAufnahme im Morgenblatt, der Allgemeinen Zeitun uufnahme im Morgenblatt, der Allgemeinen Zeitung udgl. geeignet seien. Schließlich hat er seine englischen Benutzung und zum Auszug interessant erscheinende Artikel zur Verfügung gestellit

Umgekehrt hat Johann Friedrich Cotta von sich aus auf die Dienste Johann Gottfried Dinglers zurïckgegriffen. So hat er ihn eingeschaltet und in Anspruch genom men beim Kauf desjenigen Hauses in Augsburg, in dem dann $x 824$ die erste Dampfschnellpresse Sïddeutschlands für die Allgemeine Zeitung licf, und Dingler kümmerte

${ }^{64}$ Z.B. CA I/x4 I9.x.I820; CA I/26 25.x0.1820; CA I/88 22.x0.1825; CA 1//109 22.6.I826 u

7.5.1830; $\mathrm{CA}$ I/I20 ${ }^{6.9 .1825} \mathrm{ZB}$

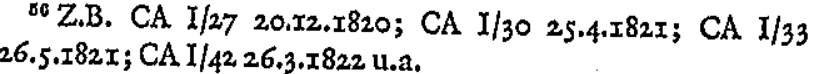

sich noch später um die Pläne und Umbauten des »neu erkauften Pallastes « ${ }^{6}$.

Nach dieser Hauskaufangelegenheit bot Dingler dem Baron von Cotta wiederholt seine uneigennützigen Dienste an: "Befehlen Ew. Hochwohlgebohren wo ich dienen kann.« Und anläßlich seiner Reise zur Sitzung der Zollkommission in München fragte er an, ob er etwas für Cotta in München besorgen solle; er erwarte dessen Befehle $e^{68}$.

Indem Dingler Cotta unterrichtete über Vorgänge und Probleme der Augsburger Cotta-Betriebe oder über den Stand von Konzessionierungen und indem er ihm Nachrichten weiterleitete über dic Stimmung und die Ansichten der Münchener Ministerialbüroktratic zu wirtschaftspolitischen Vorhaben, und da Dingler den Minister von politischen Vorhaben, und da Dingler den Minister von
Armannsperg persönlich gut kannte und König Ludwig I. großs Stücke auf ihn hielt ${ }^{50}$, war Dingler für Cotta ein unterrichteter und uneigennütziger Gewährsmann sowie ein bis zu einem gewissen Grade einflußreicher politischer Agent. Sicherlich war Dingler sen. keineswegs die einzige Nachrichtenquelle Johann Friedrich Cottas. Im Netz von dessen vielätigen Beriehungen lam ih zeitweise eine wichtige Rolle zul. So als nach der Berufung Dinglers in die Mautenkommision die Fragen der Zollpolitik, der Zollsätze und der Vorarbeiten zur Gründung des Zollvereins einen größeren Raum in der Korrespondenz einnehmen. Dingler stand hier in ständigem Meinungsaustausch mit Cotta, er hat die Interessen des Verlegers mit besonderem Eifer vertreten und hat als Kommissionsmitglied Cottn vorgeschlagen, wie dieser mit seinen Eingaben prozedieren und in dicsen taktieren sollte, um z. B. die Zollbegünstigung für das Papier herauszuholen. Diese "Papierangelegenheit" wurde denn auch, wie Dingler Cotta abschlicßend mitteilte, vom Ministerium "ganz nach Ihrem Antrage und Wunsch erledigt « ${ }^{00}$. Johann Friedrich von Cotta hat Dingler von sich aus wiederholt um Rat gefragt oder um ein Urteil gebeten über bestimmte neue Maschinen oder Verfahren, welche or eventuell für seine Wirtschaftsbetriebe anzuschaffen

${ }^{57} \mathrm{CA} \mathrm{I} / 45$ - I/55 9.10.1822 - 3.2.2.8823; CA I/57 6.4.1823; $\mathrm{CA} I / 598.7 .1823$ 11.2.

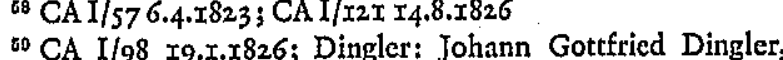
S. 730 .

${ }^{60}$ Z.B. CA I/I2I I4.8.x826; CA I/x22 27.x2.1826; CA I/x23 I6.X.I827; CA I/126 - I/x30 7.2.1/827; CA I/I82 30.I.I830; CA I/219 24.4.1832 u. a. Uber Cotta als Verfassungspolitiker und seinc Rolle bei der Gründung des Zollvereins siche auch: Albert Schäffle: Cotta (Geisteshelden $x 8$, Berlin I895), S. 8gff., I2Iff. gedachte $^{01}$. Cotta wies Dingler auch mehrmals auf technische Erfindungen und Neuerungen hin, welche in Polytechnischen Journal angezeigt werden soliten. Meist waren aber diese bereits im Journal beschrieben worden, oder der Herausgeber konnte plausible Grïnde dafir war $^{62}$

Die Verquickung der geschäftlichen mit den nicht buchhändlerischen und persönlichen Beziehungen, wic diese zum Stil im Umgang mit seinen Autoren bei Johann Friedrich von Cotta gehörte, zeigt sich auch darin, daß man gegenseitig an den Familienereignissen Anteil mam Zum Beispiel gratulierte Dingler dem Baron von aller Frauen« und die Bitte »der huldvollsten der Frauen dero Fraue Gemahlin meinen devoten Handkußu zu übermitteln, gehört üblicherweise zu den Schlußwendungen der Briefe des Herausgebers an den Verleger. Cotta wiederum sandte ein Geschenk zur "Copulation" von Emil Dingler, oder man setzte sich in Kenntnis von der Verlobung der Kinder ${ }^{83}$.

Diese unterschiedlichen und vielfältigen Beziehungen auf verschiedenen Ebenen haben sich zwischen Cotta sen. und Dingler sen. offensichtlich zu keiner Freundschaft verdichtet, wenn auch Cotta bei Dingler ähnlich wic bei seinen großen Autoren in das "persönliche Leben mit einbegriffen war und eingriff ${ }^{64}$. Selbst wenn man die Höflichkeitsfloskeln der Zeit berücksichtigt, hat man aus den Formulierungen und der wiederholten Betonung der Diensteifrigkeit den Eindruck, daß Johann Gottried Dingler viel an der die eigene Reputation erhöhenden Bekanntschaft mit Johann Friedrich Cotta lag. Die Versicherung der "Bewunderung" und sunbegrenzten Hochachtung» war für Johann Gottfried Dingler sicher keine leere Formel.

Nach dem Tod Johann Friedrich von Cottas $x 8_{32}$ verengt sich dieser, nicht das Polytechnische Journal ode die wirtschaftliche Existenz der Firma Dingler und dam Honorarforderungen bzw -überichungen betreffende Teil der Korrespondenz zunehmend auf begrenztere literarische Themen über die Auflage oder die Neuauflage von technologischen Titeeln. Die Verhältnisse kehren sich unter den andersgearteten und distanzierteren Temperamenten von Emil Dingler und Johann Georg von Cotta auch eher um. Nicht erbetene Anregungen oder

${ }_{01}^{01}$ Z.B. CA I/9 20.10.x819; CA I/35 27.8.I82I; CA I/52 I4.I.I823 u.a.

${ }^{20}$ Z.B. CAI/63 29.8.1823; CAI/65 30.10.1823.

${ }^{03}$ Z.B. CA I/70 3I.I2.I823; CA I/80 27.9.1824; CA I/I25

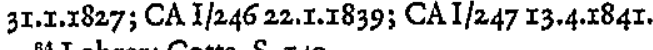

${ }^{64}$ Lohrer: Cotta, S. I40. 
Meinungsäußerungen $z u$ nichtbuchhändlerischen Themen seitens Dinglers gibt es nun kaum mehr. Vielmehr bittet dic Cotta'sche Verlagshandlung jetzt ihrerseits um die Angabe von Abhandlungen, welche zur Aufnahme in die Allsemeine Zeitung reeignet wären oder sie hol Emil Dinglers Urteil ein über die Qualität cince legenden Buches o Werk neu auflegen soll ${ }^{55}$. Es scheint, daß mit dem Tod

von Johann Friedrich von Cotta auch der intuitiv sichere Griff bei der Ubernahme technologischer Literatur in Verlagsprogramm abhanden gekommen ist. Und tatsächlich hat sich unter dem mehr der Bewahrung des Übetkommenen verpflichteten Erben Georg von Cottn der Verlag technologischer Verlag technologischicr Tite keneswegs so stürmisch Cotta

3 Die Grundlagen der Herstellung und das Erscheinungsbild des Journals

3.I Das Herausgeberhonorar

Im Gründungsschreiben vom 27. Juni I8I9 hat Johann Gottfried Dingler den vom Verleger akzeptierten Vorschlag auf ein jährliches Herausgeberhonorar von 2500 Gulden gemacht. Dafür sollte jeden Monat ein Heft zuı 7 bis 8 Bogen in Oktav erscheinen, zu denen Dingler das Manuskript, die Zeichnungen für die Kupfer und gegebenenfalls Zeugmuster $\mathrm{zu}$ liefern hatte. Von diesem Honorar mußte Dingler auf seine Kosten dic für die Herausgabe des Journals benötigte in- und ausländische Literatur besorgen und hatte die Mitarbeiter zu seinen Listen zu honorien, wie cr auch nod getegentich VerLen the unchthen sich Dingler noch 4 Gratisexemplare des Journals auf feinem und 5 auf Schreibpapier aus ${ }^{67}$.

Es war dies ein Pauschalhonorar, wie ein solches auch zu erwarten gewesen war für ein neues periodisches Unternehmen mit völlig ungewissem Ausgang. Dingler war nicht am Erfolg des Unternehmens beteiligt und hat es auch nicht angestrebt. Das größere Gewinn- und Verlustrisiko lag beim Verleger. Uar einen formlichen Vertragsabschluß ersetzende Gründungsschreiben auf dic Bogenzahl und das Format einging, war allerdings bei eincr entscheidenden Anderung der Bogenzahl oder auch des Formats billigerweise auch eine Änderung des Honorars zu erwarten.

Das Honorar wurde anfangs jährlich zu Beginn des nächsten Jahres furr das vorangegangene Jahe im 24 fl

${ }^{05}$ Z.B. CA II/9 3r.x.x837; CA II/r4 27.xr.1838; CA II/ $/ 26$ xx.8.I843; CA II/I7 I2.9.x843; CA II/32 I7.xI.I852; CA II/ 16.5.x855; CA II $/ 4324.4 .1857$; CA II $/ 46$ x8.6.x858 u.a. Lolrer: Cotta, S. I07; uber Gcorg von Cotta auch: Herbert Schiller: Georg von Cotta. Schwäbische Lebensbilder (Sutgatt 1941$), 5.54-66$.

"CA I/4 27.6.x8x9; CA I/6 8.7.x8x9; Dietze: GeschichteS. 3.
Fuß über das Augsburger Bankhaus J. \& G. W. Halde von der Cotta'schen Buchhandlung in Stuttgart fï Dingler angewiesen ${ }^{88}$,

Im Zusammenhang mit dem noch zu besprechenden tung im Jahre 1825 hatte Dingler die Absicht, sich vom Pauschalhonorar zu trennen. Er warf dic Frage an Cotta auf, nob dieselben sich in den Nuzen mit mir theilen wollen? Denn auf ein bestimmtes Honorar bin ich nicht gesonnen mich einzulaßen, weil ich einem bedeutenden Absaz, in der Art wie das Blatt redigirt wird, entgegen sehe «00. Er wollte das Unternchmen auf gemeinsame Nutzen oder Schaden von Verleger und Herausgeber betreiben.

Die Ablehnung des Zeitungsplans durch Cotta und die als Kompromiß aus den unterschiedlichen Vorstellungen von Verleger und Herausgeber zu verstehende nunmehrige zweimalige Erscheinungsweise des Journals pro Monat fühtte zum Rücktritt Dinglers von seinem ZeitungsInteresse. Der in diesen Jahren einsetzende bessere $\mathrm{Ab}$ satz ermöglichte es auch dem Verlagshaus Cotta, ihm in der Honorarangelegenheit entgegenzukommen. Sein $\mathrm{Ho}$ norar wurde im Oktober I825 auf $4000 \mathrm{fl}$ jöhrlich arhö und war jetzt vierteliahtlich an zahlen. Dingles hate

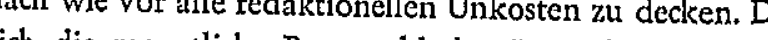
sich die mournals mit diese Anderung des Erscheinungszyklus von maximal 8 au maximal I2 Bogen erhöht hatte, war Dinglers Honorar proportional gestiegen, ja es hatte sich sogar noch darther hinaus verbessert ${ }^{70}$. Das Honorar war nach wie vor ein Pauschalhonorar, bei seiner Erhöhung sind jedoch die Vermehrung der Bogenzahl und der dam

${ }^{\circ 8} \mathrm{CAI} / 28$ ×8.x.782.

${ }^{\circ 0} \mathrm{CA} I / 85$ 7.I0.I825; CA I/85 a Oktober I825; Dictzo Geschichte, S. 5

${ }^{10} \mathrm{CAI} / 87$ 2x.ro.x825. Plan Dinglers zur Gründung einer Polytechnischen $\mathrm{Ze}$ plan und von den Ansprüchen auf ein gemeinschaftliche
Arbeitsanfall für den Herausgeber nicht unberücksichtigt geblieben.

Allerdings ist der genaue Umfang dieser Erhöhung der Herausgeberbezüge nicht unumstritten. Dietze gibt das Honorar vom Oktober 1825 mit $3000 \mathrm{fl}$ an. Dies erklärt sich aus der schlechten handschriftlichen Schreibung der cntscheidenden Ziffern 3 oder 4 und der daraus resultierenden unterschiedlichen Lcsart. $\mathrm{Da}$ aber auch Joachim Kirchner in seiner Geschichte des deutschen Zeitschriftenwesens aufgrund von Cotta'schen Verlagsarchivalien für Dingler ein Honorar von jährlich $6860,-\mathrm{M}$ in diesen Jahren angibt, was bei ciner Rückrechnung von der Mark in die Guldenwährung den $4000 \mathrm{fl}$ entspricht und sich die 4000 Gulden Honorar ab dieser Zeit auch anderweitig nachwcisen lassen, steht fest, daß I825 tatsächlich das Honorar auf $4000 \mathrm{fl}$ ethöht worden ist $\mathrm{t}^{71}$

Wegen der Darlehen und Vorschüsse Cottas an Dingler sowic der Verschuldung des letzteren ist wie schon vor $x 825$ die Praxis der Zahlungsmodalitäten komplizierter gewesen. Nicht selten hat Dingler sein Honorar gar nicht in die Hände bekommen, da er bereits vor der Auszahlung gezwungen war, Wechsel auf die Honorarverito

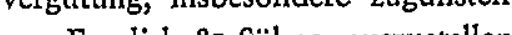
von Froelich \& Solnc, auszustellen ${ }^{72}$. Um nicht ständig in die beiderseitig unliebsame Lage zu kommen, vor der Zeit trassieren zu müssen, bat Dingler Cotta zum Beispiel, das Honorar für I829 "quartaliter" statt an ihn gleich direkt an seinen Bankier zu überweisen, dessen "Wetterfahne" sich nach der Zustimmung Cottas denn auch ein wenig nach einer freundlicheren Windrichtung drehte ${ }^{73}$. Hatte Dinger eine Vorausbezahlung des Honorars von Cotta wieder ausgeglichen, daß Dingler bis zum Ausgleich keine Honorarzahlung in Anspruch nahm ${ }^{74}$. Die vierteljährliche Bezahlung des Honorars hat sich nicht lange gchalten, denn bereits für $x 830$ wünschte Dingler die monatliche Bezahlung durch die Expedition der Allgemeinen Zeitung. Und später ist auch nur mehr von der monatlichen Zahlunosweise dic Rede ${ }^{70}$.

Von Cotn is bekannt, daß ex den Autoren und Herausgebern seiner Verlagsprodukte mit der verbesserten

"Dietze: Geschichte, S. 5; Joachim Kirchner: Das deutsche Zitschrift Gesen Seinc Geschichte und seine Probleme $x_{2} 2$ (Wiesbaden I958, I962) hier 2, S. 461f. I/I67 24.6.x828 u.a.

${ }^{73} \mathrm{CAI} / 1772$ 30.10.1828; CA I/I73 4.II.1828

${ }^{14} \mathrm{CAI} / \mathrm{xgI} 26.8 . \times 830$

${ }^{75} \mathrm{CA} \quad 1 / 174 \quad 29 . x 2 . x 828 ; \mathrm{CA} \mathrm{I} / 280 \quad 3 x .12 .1829 ; \mathrm{CA} \mathrm{II} / 36$ 3r.3.5855.
Rentabilität eines Werkes von sich aus höhere Honorarc anbot. Es verwundert daher nicht, daß Cotta 1836 , anscheinend olne vorherige Intervention Dinglers, das Honorar für das Polytechnische Journal um $500 \mathrm{fl}$ pro Jahr erhöhte; 1843 ist dann das Honorar auf $5000 \mathrm{H}$ festgesetzt worden ${ }^{70}$

Vergleicht man die Höhe dieses Herausgeberhonorars mit denen, welche für andere Zeitschriften gezahit wurden, so ergibt sich, daß die Herausgeber des Polytechnischen Journals überdurchschnittlich vergütet wurden Ohnc Zweifel hat Cotta durch die Höhe seiner Autorenhonorare erstmals die geistige Arbeit angemessen honoriert. Es sind auch Herausgeber mit Spezialkenntnissen und von Zeitschriften, die mehr Arbeit verursachten, noriert worden als Redakteure von reil eisteswisenschalichen B1ätern 77 . Bei der Einordnun des Herausgcberhonorars des Polytechnischen Journals in die Relation der sonstigen Honorarvergütungen für Zcitschriften wird man aber auch die Umstände der Entstchung und die Zusammensetzung dieses Honorars mitberïcksichtigen müssen. Das Honorar und die Honorarerhöhungen des Journals sind zunächst zu sehen vor dem Hintergrund der Auflagen- und Absatzstcigerungen, welche arkancon lasen, di das Polyteclnische Journa scheinungsweise geändert. Vor allen abcrelativiert sic die Höhe des Honorars sofort beträchtlich, wenn ma sich die Auslagen Dinglers für die Redaktion vor Augen hält.

Johann Gottfried Dingler hat schon in seinem Gründungsschreiben darauf hingewiesen, daß ihm nach Aufrechnung des Honorars yon $2500 \mathrm{fl}$ mit seinen Redaktionsausgaben bei der Herausgabe dieser Zeitschrift vermutlich kein anderer Gewinn zuteil werden dürfte als die Ehre und das Bewurksein, etwas bleibend Gemeinnütziges geschaffen zu haben. Bereits $I 825$ äußerte er sich Cotta gegenüber, daß ihm in den letzten zwei Jahren kein Nutzen an der Herausgabe des Polytechnischen Journals zuteil geworden sei ${ }^{78}$. 1836 schrieb er etwas indigniert an Cotta, als er irrtümlich der Ansicht war, daß das Honorne für den laufenden Jahrgang nur um 250

${ }^{70}$ Kirchner: Zeitschriftenwesen 2, S. 462; CA I/239 I7.xI x836; CA II/ $/ 35$ xx.2.2. 8855

S. 46Iff.; Lohrer: Cotth Sircliner: Zeitschriftenwesen 2 einer Entwicklungsgesch S. 88; Walter Kricg: Materialien zu B. Bocklet, Zürich 1953), crgab für die hier vorliegende Fragcstellung nichts, da sein Material zu sehr die Honorare schön-

geistig-bellectristischer Titel berücksichtigt.
${ }^{78} \mathrm{CAI} / 87$ 2x.ro.I825. 
erhöht werden sollte, daß diese Einschränkung "mit den warlich großen Aufopferungen die wir dem Journale widmen nicht im Einklange steht, und Sie dürfen mir aufs Ehrenwort glauben, daß dabei nichts aufgesteck wird, was ich Ihnen im Falle mit einer stichhaltigen Bewird, was ich lhnen im Falle
rechnung darlegen könnte «70.

Zehn Jahre später hat er eine solche Berechnung auf gestellt und dabei ausgeführt: Die Ausgaben für das Polytechnische Journal melrten sich ständig, und die Mitarbeiterhonorare würden steigen. Genauso müßten für Korrespondenten, Porti und Literaturbedürfnisse gröBere Aufwendungen gemacht werden, wenn sich do Polytechishe Joun der Ausgaben:

"Für eigenthümliche Arbeiten und Honorare an die Mitarbeiter, dann für die Abbildungen und für die Redaction und Druckrevision sind für den Druckbogen $f_{4}$ anzunehmen was für $\mathrm{I25}$ Bogen beträgt $\quad$ fl 5500 für Literatur

für Correspondenz und andere Porto ca. fll 250 bis 300 was zu dem Honorar von $\mathrm{fl} 5 / \mathrm{m}$ in keinem Verhältnis stehto

Er führt dann noch weiter aus, daß alle konkurrierenden Journale und Zeitschriften cinen größeren Redaktionsaufwand hätten als das Polytechnische Journal. U auf der bisherigen Höhe der Leistungsfähigkeit zu blejben, müßte daher die Redaktion "von Seiten Ihrer verehrlichen Handlung durch ein entsprechendes Honora aufgemuntert werden ${ }^{80}$. $\mathrm{Zu}$ dieser Aufmunterung ist es aber vorderhand nicht gekommen.

Inwieweit diese Kostenrechnung der Redaktionsausgaben dem Zweck der Eingabe entsprechend überzogen war, ist nicht einfach zu entscheiden. Da nach dem Untergang ihrer Firma für die beiden Dingler anscheinen keine weiteren Einnahmequellen vorhanden waren scheint die Redaktion des Polytechnischen Journals jedoch immerhin so wiel eingetragen zu haben, daß beiden Dingler ihren Lebensunterhalt aus dem geberhonorar bestreiten konnten.

An die doppelte Herausgeberscist sich das Problem ter zwischen Honoraranteils zwischen Vater und Solin Dingler. Aufgrund einer Vereinbarung zwischen Cotta und den beiden Dingler in de zweiten Hälfte des Jahres 1849 waren Emil Dingle monatlich $200 \mathrm{fl}$ als Honorar durch die Expedition der Allgemeinen Zeitung zu zahlen, wogegen er "sümtlich Kosten« mit Ausnahme der durch die Literariche An stalt und das Augsburger Oberpostamt bezogenen Lite-

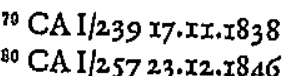

ratur selbst zu decken hatte. Seinem Vater blieb hingegen der ganze Rest des Pauschalhonorars ${ }^{81}$. Da Em Dingler damit ohnehin schon der geringere Anteil verblieb und überdies noch ein Teil der Redaktionsunkosten auf ihn entfiel, mußte er schon aus existentielle Gründen gegen das Uberziehen des Honoraranteils durch seinen Vater Front machen.

Emil Dingler war für die Cotta'sche Buchhandlung in der Honorarfrage offenkundig ein härterer und genaue kalkulierender Partner als sein in finanziellen Dingen großzügigerer Vater. Er strebte für jedes halbe Jahr cinc Neufestsetzung seines Anteils am Pauschalhonom an und arbeitete zielstrebig hin auf cine Mitbeteiligung des Herausgebers am Absatz. Seine Ausgangsposition zu einer Oberwindung des Pauschalhonorars mehr in Richtung eines Absatzhonorars war nicht ungünstig: $\mathrm{Di}$ Auflagenziffern gingen in den I85oer Jahren parallel der wirtschaftlichen Konjunktur kräftig nach oben, und der Herausgebername Dingler war inzwischen mit dem Polytechnischen Journal so eng verbu erlag praktisch unentbehrlich geworden war

Emil Dingler hat denn auch schon in den Jahren vo x855 erreicht, daß er zu den 5000 fl Pauschalhonora Zuschüsse erhielt, welche sich nach der Zahl der sei I843 alljährlich mehr abgesetzten Exemplare richteten. Da dieses Verfalren bei dem exakt die Cotta'sche Rechnungslegung überprüfenden Emil Dingler zu Irrungen and Auseinandersetumgen Anh B bot Cottn im Man Cotta im Marz 1855 folgenden, von Emil Dingler $"$ mi Vergnügen« a akzeptierten Vorschlag unterbreitet: Auf der Basis des Verkaufs von 1700 Exemplaren, wie dieser im Jahre 1854 erreicht worden war, wird als jährliches Ausgangshonorar ein Pauschalbetrag von $6000 \mathrm{fl}$ festgesetzt. Für jedes weitere zusätzlich verkaufte oder unter dieser Zahl bleibende Exemplar waren aber of auf de Pauschalbetrag anzurechnen oder abzurion

Damit hat sich das Herausgeberhonorar des Polytechnischen Journals von der Pauschalbezahlung zum Absatzhonorar hin entwickelt. Der Herausgeber partizipierte nun, ausgehend von einem an einc bestimmte Absatzzahl gebundenen Grundhonorar, an der positiven oder negativen Absatzentwicklung

\subsection{Die Grundsätze der Schriftleitung}

Mit der Zielsetzung des Polytechnischen Journals ist bereits die Richtung aufgezeigt worden, in welcher die Re-

${ }^{81} \mathrm{CA} I I / 2726 . x .785 x$

${ }^{82} \mathrm{CAIII} / 35$ II.2.2. 8855 daktion ihre Themenauswahl zu treffen beabsichtigte und für welche Leserkreise das Journal gedacht war. Hier sollen nun dicjenigen Grundsätze der Redaktio ar bestimmen Schriftleitung gegeben hat, wurden diese "Grundsätze“ für die Bearbeitung des Journals gewonnen aus den vereinzelten Eigenaussagen in den Briefen und aufgrund der Ergebnisse der Durchsicht der einzelnen Bände der Zeitschrift.

Einer der wichtigsten Grundsätze, der die Herausgeber des Polytechnischen Journals bei ihrer redaktionellen Arbeit leitete, war, dic "Icser jedesmal so schnell als möglich mit den neuen technischen Erfindungen und Entdeckungen der Alten sowohl als der Neuen Welt bekanntzumachen ${ }^{84}$.

In der Schnelligkeit der Nachrichtenvermittlung und der Bewahrung des Vorsprungs, den das Polytechnische Journal darin besaß, sah Dingler eine der Hauptvoraussetzungen für den publizistischen Erfolg des Journals. Er unt inte deme er immor che gelassen, solnge thnen gegenüber sein Journal in der Übersetzung und Ubermittlung des Nachrichtenstoffes einen erheblichen zeitlichen Vorsprung be $\mathrm{saß}$. So mcinte er I822 "In Hinsicht der Neuheit ist das polyt. Journal vor allen andern stets um 6-8 Monate voraus", "die polyt. Jahrbücher sind um $x^{1 / 2}$ bis 2 Jahre zurück « ${ }^{85}$. I 826 und 1832 äußerte er sich ähnlich, »da dic Geverbsve undlungen sow nische Jahrbücher in allen wesentlichen Sachen stets um 2 bis 3 Jahre zurück sind, während das polytechnische Journal stets à jour ist «86. Johann Gottfried Dingle wurde gegenïber seinen Konkurrenzblättern stets dan nervös, wenn er den Eindruck hatte, da $ß$ ihm auf diesem Felde seines redaktionellen Konzepts ein neues Periodikum mit Erfolg nachfolgen könnte, indem wandere polyt. Zeitschriften .... die auslandischen Journale eben so loits lom ... dic schnell kom ... dis and ine polyt. Journal zuent than, ja diese dartberhinaus "noch andere Zeitschriften kommen " lassen, "die das polyt. J. nicht berücksichtigen konnte « ${ }^{87}$. So meinte er 1826 , ein von einem sogenannten Ubersetzungs-Komptoir in We mar herausgegebenes Handwerksblatt »könnte dem po-

${ }^{84}$ Börsenblatt für den Deutschen Buchhandel und für dic mit ihm verwandten Geschäftszweige Jg. I (Leipzig I834) $\mathrm{Sp} .27 \mathrm{x}$.

${ }_{85}^{\mathrm{Sp}} \mathrm{CA} \mathrm{I} / 48$ 6.x2.x822; Dietze: Geschichte, S. 5 .

${ }^{60} \mathrm{CAI} / \mathrm{I2I}$ I6.I0.I826; $\mathrm{CAI} / 2 \mathrm{2I} 723 . \mathrm{T} . \mathrm{x} 832$

${ }^{37} \mathrm{CAI} / \mathrm{I} 9423.9 . \times 830$ lyt. Journal sehr schaden", wenn es einen der Sache gewachsenen Redakteur gewinnen könnte ${ }^{89}$. Die Notwendigkeit der Schnelligkeit der Nachrichtenvermittlung für scin Journal erkjärte er aus dem Bedürfnis sciner Leserkreise; denn für einen "Fabrikanten und Gewerbsmann" sei »die möglichst höchste Schnelligkeit der Bekanntmachung einer Entdeckung oder Etfindung" unbedingt erforderlich. "Ein Gewerbsmann, der dem andern um I4 Tage voraus ist, hat seine Rivalen vielleicht auf $x_{4}$ Monate voraus überflügelt ${ }^{8}{ }^{8}$

In der Tat ist das Polytechnische Journal, mitbedingt durch seine monatliche, dann I4tägige Erscheinungsweise, erstaunlich schnell in der Berichterstattung über die technischen Fortschritte des Auslandes gewesen. Dingler selbst meinte, daß durch sein Blatt Deutschland win längstens 4-6 Wochen" die technischen Neuigkeiten aus London und Paris erfuhrs ${ }^{80}$. Tatsächlich sind in ihm wicderholt bereits im Monat des Ersterscheinens Artikel aus ausländischen Zeitschriften in Übersetzung abgedruckt worden. Normalerweise vergingen aber etwa ein Monat oder auch mehrere Monate, ehe ein ausländischer Zeitschriftenufotz im Polytechischen Journal erschien. Dic Redarion hat deses Tempo der Obeme rend des Berichtszeitraumes im wesentlichen halten können. Es sind allerdings in den späteren Jahrgängen häufiger als früher mehrere Monate zwischen dem Ersterscheinen und dem Wiederabdruck im Polytechnischen Journal verstrichen. Ein Umstand, der nicht weiter verwunderlich ist, da die auszuwählende Literatur mit den Jahren erheblich anschwoll. Trotz der bemerkenswerten J chelligket dor wiesen werden, daß z. B. vereinzelt bei englischen $\mathrm{Pa}$ tenten bis zu zwei Jahre von der Patenterteilung bis zum Abdruck im Polytechnischen Journal vergangen sind ${ }^{00}$.

Nach der Schnelligkeit maß die Redaktion höchste Bedeutung bei der "größtmöglichsten Mannichfaltigkeit in den behandelten Themen und der Vollständigkeit in der Bekanntmachung aller Erfindungen, wwelche in Engand, Frankreich, Amerika, Italien etc. im Felde der Poyytechnik zu Tage gefördert wurden «. Schon $x 823$ hatte Dingler an Cotta geschrieben, daß er keine Gelegenheit vorbeigehen lasse, "das polyt. Journal mit den interessantesten Nachrichten zu bereichern und jedes Heft für Leser aller Stände interessant zu machen « ${ }^{01}$

${ }^{83} \mathrm{CAl} / 104$ 20.3.1826

${ }^{B 0}$ Polyt. Journal 30 (I828), S. III.

${ }^{20}$ Anstelle weiterer Nachweise siehe z.B. Polytchnische Journal 3 (I820), 55 (x835), 95 (I845) und I23 (I852).

(I) CA 1/106 I0.4.r826; CA 1/65 30.10. 823; Börsenblatt (I834), Sp. 27I; Dictze: Geschichte, S. 5. 
Zur Erreichung der größtmöglichen thematischen Vieltalt war die Redaktion bestrebt, die gesamtc ncue und neucrschienene Literatur, besonders die Zeitschriften, für uch die Mitarbeiter von Originalaufsätzen nicht außer acht. So äußerten sich Dingler sen. und jun. wiederholt zuversichtlich über den zu erwartenden besonderen Absatz dieses oder jenes Bandes, da neuc Werke bei der Themenauswahl berücksichtigt worden seien oder »dieser Jahrgang ... eine Menge Original Aufsäze « bekäme ${ }^{92}$. $x 828$ schrie Dingler sen. "Der Jahrang 5829 des polytechnischen

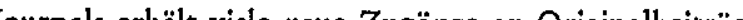
. Sachkenner fast das unglaubliche geleistet worden, so wird der Jahrgang $x 829$ durch die getroffene Vorsorg für das Publikum noch mehr leisten. $\otimes^{03}$

Inwieweit allerdings die als Ziel angestrebte Vielfalt der angesprochenen Themen erreicht wurde und inwieweit dic Originalbeiträge den Inhalt des Journals sachlich bestimmten, wird weiter unten bei der In als作 crprüft und ausgeführt.

Der dritte wichtige Maßstab der Schriftleitung war die sogenannte Gemeinnützigkeit der behandelten Themen Neben ncuen Mitarbeitern oder neu berïcksichtigte Literatur soliten vor allem "gemeinnützige Abhandlungen ${ }^{03}$ den Absatz steigern helfen. Damit war ausge drückt, daß das Polytechnische Journal als privates n der im offentlichen Interesse liogenden (orrerum ing sich bemilh Wustric. All was an literarisch verwertbaren neuen Werkstatterfahrungen und an anwendungsbezogenen wissenschaftlichen Erkenntnissen anfiel, sollte gebracht werden - und zwa derart, daß die Berichterstattung den wissenschaftlich geschulten Techniker genauso wie den cinfachen $G$ werbsmann anzusprechen und ihm etwas zu geben mochte.

Emil Dingler bemühte sich denn auch aus gegebenen Anlaß, einen Artikel iso abzufassen, daß er auch dem nichttechnischen Publicum interessant und klar sey nußs " ${ }^{24}$, Es war ein Hauptverdienst der Redaktionsarbe der beiden Dingler, ihrer Leser-Zielgruppe entsprechend, in der Auswahl der Aufsätze die Mitte und ein ause

02 Z.B. CAI/98 I9.x.r826; CAI/ISI 22.IT.x827. ${ }^{03} \mathrm{CA} 1 / 1 / 72$ 30.x0.1828; CA I/x74 29.12.I828; CA II/S7 15.2.I862 letztere Zitatstelle mit dem Hinweis auf den nich eingetretenen Erfolg im Absatz wogenes Verhältnis gehalten zu haben zwischen den wisenschaftlich-vertieften und den leichter verständlichen theorieärmeren Axtikeln. Das große Problem der Herausceber des praxisbezogenen Polytechnischen Jourma war dabei, nicht nur die zu sehr theoretisierende "kopflastige « Zeitschrift, sondern auch, daß sie aufdringlichen "Erfindern", unnützen Erfindungen oder technischen Hirngespinsten aufsaßen. Thre gediegene technisch-naturwissenschaftliche Bildung hat Vater und Sohn Dingle vor der letzteren Gefahr bewahrt. Die beiden Dingle lehnten daher die Aufnahme von Beiträgen ab, wenn dic plikationen goutierten sie ebenfalls besonders dan nicht, wenn die Originalaufsätze bercits im Journal gebracht worden waren ${ }^{05}$.

In einem Fall erklärte Johann Gottfried Dingler klipp und klar, "daß sich das polyt. Journal nicht als Vertheidiger einer so zweifelhaften allen Gesezen der Physik widerstrebenden angeblichen Erfindung hergeben und somit den bisher aufrecht erhaltenen Credit muthwillis oder wissenschaftslos hergeben könne «00. Die Herausgeber verlangten von Mitarbcitern eine »sachverständige Feder ", kein "Gefasel « und gingen z. B. bei einem Autor, "der schäzbare practische Abhandlungen« lieferte, abe bei dem es in der Theorie haperte, mit dessen Einverständnis dazu über, dessen Artikel nicht nur zu redigieren, sondern völlig umzuschreiben ${ }^{27}$. Durch dit Tafeln und die Beigabe von Anmerkungen und oft mehrsitigen Zusätzen von seiten der Herausgeber und der Ubersetzer, dann durch auszugsweise Übersetzungen, Zusammenfassung der Ergebnisse oder leichter verständliche Kürzungen von langen oder komplizierten Originalartikeln suchte die Redaktion nicht sclten den Anwendungs bezug und den Lchrstoffcharakter des Inhalts stärker in den Vordergrund zu rïcken und machte so das Jour lesbar. Sie verwies a hä hor auf gleche oder lesbar. Sie vcwics auch hautig auf gleiche oder ähnlich Artikel und schon bekannte Verfahren. Immerhin is dem Polytechnischen Journal $x 8_{34}$ der Vorwurf gemach worden, es menthalte zu Viel nur für England Anwendbares $\aleph^{08}$

In Schnelligkeit, thematischer Vielfalt und Gemeinnützigkeit hat man die drei wichtigsten

${ }^{03} \mathrm{CA} I / 65$ 30.T0.1823; CA I/I69 4.7.x828; CAII/2 2.x.x834 CAII/9 2T.x.I837.

${ }^{07}$ CA I/I20 6.9.1826; CA I/255 23.I2.1836; Dietze: Gcschichte, S. 4 .

${ }^{08} \mathrm{CA} \mathrm{II} / 3$ 20.2.I834; Börsenblatt $x$ (I834) Sp. 27x; Dietze: Geschichte, S. 6 .
Maximen der Herausgeber zu sehen. Ihnen gegenüber treten alle anderen Richtlinien an Bedeutung zurück, sic hatten auch kaum einen wesentlichen Einfluß auf den Erfolg des Journals. So litt Johann Gottfried Dingle unter dem Nachdruck von Aufsätzen oder Ubersetzunan dem Polytechnischen Journal, ohne daß dic Qun drastisch Maßnahmen durch Anprangerungen solcher Plagiate in Polytechnischen Journal ergriff(00. Sein ihn ehrendes Grundprinzip bei der Herausgabe des Journals war, zu allen Beiträgen die Herkunftsangabe zu liefern, was $\mathrm{e}$ mit zufälligen Ausnahmen auch durchhiclt.

An andere Aussagen über seine Redaktionstätigkeit z. B., daß er keine Abhandlungen in Fortsetzungen anB. Dic

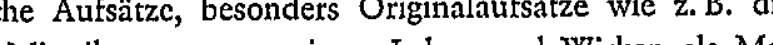
"Mitteilungen aus meinem Leben und Wirken als Maschinenbauer" von Dr. Enst Alban, zichen sich wic wahre Lindwürmer durch eine große Zahl von Hefte des Journals.

3.3 Druck und Erscheinungsweise des Journals

Der Rückgang des Anteils der zwanglos und unregelmäßig erscheinenden Zeitschriften und der Ưbergang $z$ regelmäßiger periodischer Exscheinungsweise, der in enger Beziehung steht zum technischen Fortschritt au dem Gebiet der Herstellung und des Vertriebs, ist ei wichtiger Zug der Entwicklungsgeschichte der Zeitschrifin 2 . Jahldunde1 ${ }^{101}$ Gegen die von Dingler in seinem Gründungsschreiben vorgeschlagene monatliche E scheinungsweise der Hefte des Polytechnischen Journal hatte Cotta keine Einwendungen erhoben, und die Zeitschrift erschien in den nächsten Jahren in jährlich zwöl Heften zu mcist je 8 Bogen. 4 Hefte ergaben einen Band so daß pro Jahrgang 3 Bände herausgegeben wurden. Dic termingerechte Fertigstellung der Zeitschrift war in hohem Grade abhängig von der Leistungsfähigkeit der Druckere und wude daduch erlechtert $\mathrm{d} B \mathrm{~B}$ der Dinckere und we daduch eleicher da ReEs ist oben bereits darauf hingewiesen worden, welchen

${ }^{n 0}$ Z.B. Polyt. Journal 30 (x828), S. III; Polyt. Journal 36 (I830), S. 333 ff.; 44 (I832), S. 236f.; 7 I (x839), S. 484; Börsenblatt $I$ ( 1834 ), Sp. 27 If.

${ }^{100} \mathrm{CAI} / 4 \mathrm{I}$ 30.x.I822; Dietze: Geschichte, S. 4

${ }^{101}$ Gerhard Menz: Dic Zeitschrift. Ihrc Entwicklung un ihre Lebensbedingungen. Eine wirtschaftsgeschichtliche Studie. Stuttgart $x 928, S$. 7 Iff. hohen Wert Dingler dem Druck des Polytechnischen Journals in Augsburg anstatt in Stuttgart beigemessen hat und $d a ß$ es darüber bcinahe zum Bruch zwischen Herausgeber und Verleger gekommen wäre ${ }^{102}$. Während des gesamten Untersuchungszeitraumes wurde das Journal gedruckt in der "Druckerei der J. G. Cotta'schen Buchhandlung, Augsburg«

Ab Band I9 (Jahrgang I826) ging man als Ergebnis des Kompron Ion Verleger und Herausger mech der Ablehnung des Dingler'schen Zeitungsplanes durch Cotta zur halbmonatlichen Erscheinungsweise über. Dingler schrieb damals an Cotta: $\gg D$ a sich Dieselben bei diesem Journal von Form und Zeit so ungern trennen, so könnte das Auskunftsmittel 2maligen Erscheinens in einem Monat geeignet seyn, die Interessen zu vereinigen. Das erste Heft wäre am r. u. das 2te am I5. Januar auszureben; und in der Druckerci die sichere Veranstaltung 2u unften, da $\mathrm{die} Z$ Zitordung eingelalton wird. Mo2u atich wầcn denn 2, 3 und im Nothfall ausnahnsweise 4 Bogen mehr zu geben als bisher. Jeder Band umfaßte jetzt 6 Hefte zu anfangs meist je 6, später fast durchwegs 5 Bogen. Pro Jahrgang erschienen nun 4 Bände des Journals. Diese rasche Erscheinungsfolge ist ermöglicht worden durch den Referatecharakter des Organs. Das Polytechnische Journal gehörte damit unter den technisch bemerkenswerten Blättern zu denjenigen it der dihesten Erscheinungfolge. Doch ist dis ErDiteln von fast zwei Dritteln um die Jahrhundertmitte eine Eigentümlichkeit der Polytechnischen Blätter gewesen ${ }^{104}$. Der Ubergang zu vierzehntägiger Erscheinungsweise ab x826 mag Cotta unter betricbswirtschaftlichem Gesichtspunkte erleichtert worden sein, das er gerade damals in Jahre 1824 die seine Druckkapazität ausweitende und beschleuniende Schnelldruckerei in Augsburg in Betrieb genommen hatte, in welcher auch das Polytechnische Journal gedruckt werden sollte ${ }^{105}$.

Die konkreten Einzelprobleme im Zusammenhang mi dem Druck und der Erscheinungsweise knüpften in de Regel an den nicht rechtzeitigen Druckabschluß bzw. die nicht eingehaltene termingerechte Ausgabe des Journals an.

Hatte sich schon der Druck und die Auslieferung des ersten Heftes des Journals im Jahre $x 820$ entgegen der Absicht des Herausgebers bis weit in den Jänner hinein verzögert, so tauchte dieses Problem besonders zum Jah-

2 Sielc oben Kapitel 2.

104 Schulz: Fachpresse, S. 44, 47 f

${ }_{105} \mathrm{CA}$ I/8x I4.x.I825. 
reswechsel auch bei späteren Jahrgängen gelegentlich auf. Speziell die nicht rechtzeitig gelungene Fertigstellung der Register oder der Tafeln haben an diesen Verzögerungen meist ihr gerüttelt $\mathrm{Maß}$ Anteil gehabt.

War es in den ersten Jahrzehnten eher Dingler, der Cottn dion in de ken daß diese ken, daß diese Vokehrungen trelle, um das Polytechnsche Journal termingerecht fertigzustellen ${ }^{100}$, so wollt nach 1850 eher der Verleger die pünktliche Einhaltun der Erscheinungstermine gesichert wissen. Vor allem wa ihm an einer pünktlichen Versendung der Hefte gelegen. So sollte Ende 1850 das crste Jännerheft für $185 x$ noch vor Weihnachten zum Versand kommen, dem das zweite Dezemberheft I850, das wegen des Reristers mehr A das Polytechnische Centralblat mit Verspätungen beim Abonnenten eintrafen und Emil Dingler seinerseits immer wieder eine pünktliche Erscheinungsweise als sein wichtiges Anliegen bezeichnete, verwahrte er sich 1862 gegen den Vorwurf des Verlages, einen geringfügigen Absatzrückgang einzig dem Umstan zuzuschreiben, daß zu Beginn des Jahres mit dem 2 Dezemberheft nicht zugleich das I. Jännerheft verschich worden sei. Emil Dingler thate auch dos Ansinen , eine zu möglichen Mißdeutungen Anla gebende Erklärung in das Polytechnische Journal einzurücken, nach welcher er sich fortwährend bemühen würde, das Journal zweckentsprechend auszustatten ${ }^{105}$ Hauptziel der verschiedenen Auscinandersetzungen und Bemühungen um ein fristgerechtes Erscheinen des Jour nals war dabei, das 2. Dezemberheft und das I. Heft des nächsten Jahres noch yor dem Ende des Jahres auszulie fern oder aber das $x$ Jünerheft zumindest in der $x$ Jannerwoche zum Versand zu bringen.

EXKURS: DER ZEITUNGSPLAN

Johann Gottrried Dingler unterbreitete dem Baron von Cotta am 7. Oktober 1825 den Plan zur Umwandlun des Polytechnischen Journals in eine tölich ersche "Polytechnische Zeitung (oder Allsem Fabrikanten und Gew (Oder Aly Dic Zeitungelan war Dinger au diesen Zegun legungen gekommen: Fortschrittsgläubig wie er war, wa er zunächst einmal überzeugt, daß alle neuen Ideen und

\section{${ }_{100}^{100}$ Z.B.: CAI/44 2,6.x822.}

${ }^{x 08} \mathrm{CA}$ II/29 2.xx.I85I; CA II/60 8.4.x862; CA II/62 xx.xx.
Erfindungen auf schnellstem Wege den Fabrikanten und Gewerbsleuten zur Information und zum möglichst schnellen Nachvollzug in der Praxis zugänglich gemach werden mußten. Die Fülle der Erfindungen und EntdekJungen - das hatte ihn die bisherige Erfalurung mit de

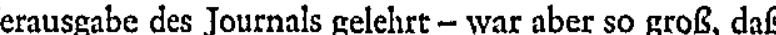
diese das Polytechnische Journal selbst bei überschrittener Bogenzahl bei weitem nicht zu fassen vermochte. Die monatliche, gelegentlich noch durch den Versand verzögerte Erscheinungsweise des Journals entwertete den Neuigkeitswert der Nachrichten durch Vorinformationen in den Zeitungen und durch Veraltetsein erheblich. Ein weiteres, in seinem zweiten Plan von $I 830$ hervorgehobenes Argument für eine ung war, daß amit scinen publizistischen Konkurrenten, die von ihm die Schnelligkeit und die Vielfalt in der Übermittlung des technischen Nachrichtenmaterials übernommen hätten und die teilweise bereits Zeitungen für cinzelne $Z$ weige der Industric herauszugeben im Begriff seien, das Wasser abgegraben werden könnte. Durch eine Auswertung weiterer Zeitschriften, die bisher wegen des Platzmangels im Polytechnischen Journal nicht mölich sewen sollte die Infornationsbrete der au gründenden Zeitung his zur Vollstundigkeit ausgeweitet werden. Scin Sohn werde ihn bei der Redaktion unterstützen. Im unklaren war sich Dingler noch, ob diese Polytechnische Zcitung mit der Allgemeinen Zeitung verbunden werden oder ob sie voliständig getrennt von dicser erscheinen sollte. Möglicherweise sollte sie auch den Abnehmern der Allgemeinen Zeitung wohlfeiler abosen werden

Nach dem von Dingler an Cotta eingesandten spezifizierten Vertragsentwurf solite in der Druckerci alle zwe Tage ein Bogen gesetzt werden für zwei Nummern der Zeitung, deren Ausgabe dann täglich erfolgen sollte. Monatlich sollte eine Inhaltsanzeige beigegeben werden. Die Zeitung sollte - wie bereits bei der Besprechung des Honorars ausgeführt - auf gemeinsamen Schaden und Nutzen von Verleger und Ober Ausgen Buch führen. Anzeigen in und von Cotta'schen Druckwerken sollten gegenseitig nicht berechnet werden. Der Herausgeber wollte für dic Ausgaben zur Beschaffung der Literatur, der Honorare, Übersetzungen, Korrespondenzen, Zeichnungen $\mathfrak{u}$.dgl. aufkommen, wofür ihm jährlich $4000 \mathrm{fl} \mathrm{zu}$ vergüten waren. Papier, Druck, Kupferstich und Kupferdruck sowie die Versendun hatte die Vetlagshandlung zu besorgen. Neben einigen

${ }^{100}$ Zum Zeitungsplan siehe besonders CA I/85 7.ro.r825; CA I/85a Oktober 1825; CA I/x94 23.9.1830; Dictzc: Ge-
schichte, S. 5 weiteren Einschüben wie Vertretung des Herausgebers im Krankheitsfall, ciner Bestimmung über die Eigentumsrechte des Herausgebers im Todesfall und der Werbung für die Zeitung vor deren Ersterscheinen sollten sich Gerausgeber und Verleger mit den deutschen Regierun-

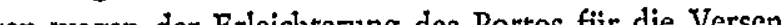
gen wege der Breich so billig als thunlich gestellt werden, damit diese Zeitung den Gewerbsleuten, für die sie bestimmt ist, auch zugänglich wird. «110 Wie Dingler in dem Brief schrieb, in welchem er Cotta den Zeitungsplan zum ersten Mal entwickelt hatte, wäre der höchste Preis der Zcitung mit Ix Gulden zu veranschlagen. Ein Teil der Kosten würde durch Avertissements redeckt werden können. Für die Kup in Quart wire in Kuper in Quart wire ein ciganer supferdn astellen. Sollo Cotta die Unent würde Dingler sie selbst für seine eigene Rechnung einleiten.

Cotta sagte die Unternehmung in der Art wie sie Dingler vorschlug, tatsächlich nicht zu. Die einzelnen Argumente sciner Ablehnung sind nicht bekannt. Hauptgrund dürfte gewesen sein, daß er sich von der täglichen Erschcinungweise nichts versprach. Im zukünftigen Bogench Joul Ding des Jouls bisherigen 7 bis 8 Bogen monatlichen Umfangs und einmaliger monatlicher Erscheinungsweise soilte ja nun nach Cottas Kompromißvorschlag an Dingler das Polytechnische Journal halbmonatlich mit 2, 3 und notfalls Bogen mehr pro Monat wie bisher erscheinen. Das heißt die Bogenzahl des Polytechnischen Journals konnte ab I826 von maximal 8 auf maximal I2 Bogen pro Mona

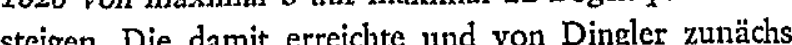
ch. Die damit err mal $x_{44}$ Boge im Jahr lag gar nicht so weit entfernt von den I57 Bogen jährlich, welche bei einem Umfang von einem halben Bogen pro Tag die Polytechnische Zeitung nach eine Rechnung von Cotta im Jahr gefordert hätte ${ }^{110}$. Da de Preis des Journals trotz der vermehrten Bogenzahl und der verdoppelten Erschcinungswcise der gleiche blieb und Dingler eine Honoraranhebung von 2500 auf 4000 zurebillit benm, war Cotta Dingler wohl schon bis a ue die Gren des with Maßes entgegengekommen. Dies um so mehr, da das Polytechnische Journal erstmals $x 824$ nachweislich $m$ Gewinn abgeschlossen hatte 111

$10 \mathrm{CAI} / 85$ a Oktober $\times 825$

. nalic Cottas auf dem Brief CAI/ $/ 85$ a Oktober 1825
Gegen die Umwandlung des Polytechnischen Journals in eine polytechnische Zeitung sprach auch, daß die stechnischen Neuerungen ... keine Tagesereignisse sind, "dic am kommenden Tage schon wieder durch neue Geschehnisse überholt sind «112. In der Praxis de Polytechnischen Zeitung hätte dies auch nur bedeutet, daß Eufindungen und technische Nachrichten aus Zeit1 . chesthritten u. dgl., die schon wegen ihre langun Enscheinungsweise keinen Tagesneuigkeit wert mehr besaßen, in der Form einer Tageszeitung verbreitet worden wären. Es muß auch bezweifelt werden, ob gerade die von Dingler als Abnchmer ins Auge gefaßten Fabrikanten und Gewerbsleute die Zeit gefunden hätten, zusätzlich zur normalen Tageszeitung noch täglich eine polytechnische Zcitung zu Iesen.

Johann Gottfried Dingler betrachtet diese am WiderJand Cottas cinc Tare Chance und griff in einem Brief an Baron von Cotta im Jahre $x 830$ nochmals ausführlich den Zeitungsplan auf ${ }^{113}$.

Dieser Plan stand jetzt stärker unter dem Gesichtspunkt der Ausschaltung der die Zcitungsidee bereits realisierenden Konkurrenz, besonders im gewerbereichen Sachsen. Dingler wiederholte das Argument, daß das

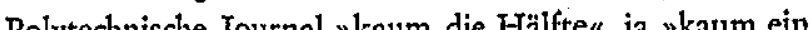
Ditteln der jathen Erfindungen tassen konnte. Nicht nur im Titel, sondern auch im Vertrieb sollte diesc »Allgemeine Zeitung für Künstler und Handwerker" Anlehnung suchen an dic Allgemeine Zeitung. In einem Intelligenzblatt nach dem Vorbild französischer technischer Journale sollte gegen eine mäBige Insertionsgebühr den Künstlern und Fabrikanten Gelegenheit gegeben werden, ihre Arbeiten, Verbesserungen und Erfindungen bekannt zu machen, wodurch fiit den Verag Risito und Unkosten sinen wirden. Dieser Zeitung, welche »die Erfindungen des Inlandes und des Auslandes schnell und vollständig" mitteilen würde, könnte "mit Benüzung der gleichen Literatur eine für Pharmacic und Chemie folgen, und nach dieser noch zwei andere naturwissenschaftliche". Eine Äußerung Cottas zu diesem zweiten Plan ist nicht bekannt. Johann Gottfried Dingler unterschätzte bei diesen Zeitungsplänen doch wohl insgesamt die anschwellende Flut technischer und naturwissenschaftlicher Erfindungen, welche

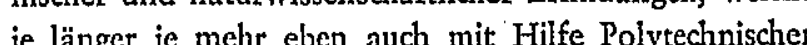
je länger je mehr eben auch mit thife Polytechnischer

\section{${ }^{113}$ Dietze: Geschichte, S. 5.}

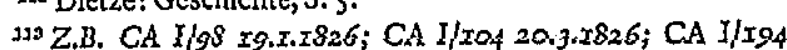
23.9.1830. 
kommen waren. Entweder man erfaßte nur mchr eine Auswahl oder erstrebte Vollständigkeit nur für cincn engeren fachspezifischen Bereich.

\subsection{Aufmachung und Ausstattung der Zeitschrift}

3.4.I Format, Umfang und Zählung

Beim Polytechnischen Journal, das in Heften ausgeliefert wurde, handeit es sich nicht um Verleger-, sondern um Buchbindereinbände. Formatangaben der Buchdeckelhöhen kömnen daher nur Aussagekraft beanspruchen fü den jewcils untersuchten Band. Ausgehend von diese Einschränkung läßt sich feststellen, daß bei den untersuchten Bänden die Höhe des Buchideckels von $20,8 \mathrm{~cm}$ mersen $\mathrm{Band}$ bis auf $22,5 \mathrm{~cm}$ im $\mathrm{B}$ is Die n ist. Die Hohe des Polytechnischen Journals war also während des gesamten Berichtszeitraumes Oktav. Es ho sich damit positiv ab von den kleinformatigen Blättern des I8. Jahrhunderts und war I8I9 auch größer als da Format der Allgemeinen Zeitung ${ }^{114}$.

Exaktere Angaben, da sich hier keine »Irregularitäten durch Buchbindercinbände crgeben, lassen sich zum Satzpiegel machen. Is werden daher im folgenden die Maße er Satzspiegel ciniger Bändo in and Höhe und zusätzlich in der Zahl der Zeilen für dic rundschrift pro Kolumne mitgeteilt

Band r: $8,9 \times x 6,8 \mathrm{~cm}\left(34\right.$ Zeilen), Band $x_{4}: 9,4 \times$ $x 7,6 \mathrm{~cm}$ (39 Zeilen), Band 2I: 9,2 $\times 18,2 \mathrm{~cm}$ (4I Zeilen) Band 40: I0,I $X$ I9,0 cm (43 Zeilen), Band 90: I0,I $\times$ I9,0 cm (43 Zeilen), Band 99: I0,6 6 I8, $2 \mathrm{~cm}$ (4x Zeilen), Band xog: I0,5 $\times$ I8,4 cm (4I Zeilen), Band I38: I0,6 $\times$ I8,3 cm (4x Zeilen) ${ }^{115}$.

Wesentlich für das Erscheinungsbild des Journals is dabei, $\mathrm{da} \beta \mathrm{ab}$ Band 99 im Zuge einer umfassenderen Umstellung in der Aufmachung des Journals bei gleichbleibender Formathöhe die Zcilenzahl pro Kolumne verringert wurde, so daß sich ein freundlicheres und auf gelockerteres Satzbild ergab.

Erheblichen Schwankungen ist der Seitenumfang des Polytechnischen Journals unterworfen höchste Seitenzahl mit selten unter 500 , mist aber is mat 600 seiten pro Band singen, colch das gingen, erreichte das Polytechnische Journal bis zum Jahre I827. Bis zum Jahre 1825 ist dies dic Zeit, in welcher nur drei Bände pro Jahrgang erschienen. Dic vie

\section{${ }^{114} \mathrm{CAI} /$ II 27.Ir.I8I9:}

${ }^{116}$ Siehe die jewciligen Bände des Polytechnischen Journals zchntägige Erscheinungsweise des Journals und die damit erreichte Zahl von 4 Bänden pro Jahr führte zunächs nicht zu einer Verringerung der Seitenzahl. Im Gegenteil steigerte vielmehr Dingler in den ersten Bänden des Jahres $I 826$ die Seitenzahl bis nahe an 600 Seiten. Wic cr an Cotta schrieb, hatte er damals einen großen Vorra an nutar Vercor auch noch nachträglich dic Notwendigkeit zur Erscheinungsverdoppelung und Bandvermehrung demonstrieren. $\mathrm{Ab}$ I828, zunächst noch mit Ausnahmen, später immer regelmäßiger, jedoch ebenfalls nicht ohne kleinere Seitenvermehrungen bei einzelnen Bänden umfaßte der Band des Polytechnischen Journals rund XII Seiten Inhaltsverzeichnis und 468 Sciten Text Bei 6 Heften pro Band zu je 5 Bo Sciten ergab das 480 gezählte Seiten. Finc Ausnahme mit etwas höherer Seitenzahl bildete dabei nahezu regelmäßig der jeweils vierte Band des Jahrgangs, in welchem das Register erschien.

Die Zählung des Polytechnischen Journals ist insofern etwas verwirrend, als die Herausgeber einerseits dic Bände fortlaufend numcrierten. Sie gaben aber andererseits ab Band 50 dem Journal gleichzeitig ein zweites Titelblatt, auf welchem sie mit dem Zusatz »Neue Folge die $Z$ ählung wieder mit Band I begannen. $A b$ Band ror lautete die Zählung auf dem linken Titelblatt "Dritte Reihc. Erster Bandu. In einem etwas verschlungene Gedankengang fühlten sich die Herausgeber zu diesem Verfahren bewogen, sum den neueintretenden Abnehmern den Eintritt zu exleichtern, der gegenwärtig, wo mehrere der früheren Bände unseres Journals gänzlich vergriffen sind, wo deren Anzahl bereits so hoch angewachsen ist, manchem Techniker auf cine unangenchme Weise erschwert ist«117.

\subsubsection{Papier und Schrift}

Die im Zusammenhang mit dem Papier zwischen Herausgeber und Verleger auftrctenden Probleme ribren fast durchwegs daher, daß das Papier des Polytechnischen Journals zu wenig weiß war und vor allem, daß zu zunnals zu wenig weiß war und vor allem, daß auf die an war. Der Druck schien von einer Papienseitc Johann Gottfried Dingler war dabei auch besorgt, da die einzelnen Journalhefte zu dünn wïrden Bindruck der Hefte beim Publikum abtiglich ware

${ }^{110} \mathrm{CAI} / 96$ 26.12.x826.

Borsenblatt $x(x 834)$, Sp. 272 .

5.x2.x845.
Es entsprach daher einem alten Wunsch der Redaktion, als sich der Verleger entschloß, mit dem Jahrgang 846 (Band 99) dem Journal eine bessere Ausstattung zu geben und dabei auch weißercs und dickeres, nicht meh so durchscheinendes Papier zu verwenden. Der Wunsch von Dingler sen., daß das Papier in Zukunft in der Qualitit litat gleich bleiben mese denn bereits x8s daß beim Papier des Jounals haufing zu dünne Papie bogen vorkämen, so daß sich die meisten Lescr eher ei weniger weißes, dafür um so stärkeres Papier wünsche dürften ${ }^{110}$. Uberhaupt war es auch schon bei früheren Bänden wiederholt vorgekommen, wenn die normale Papiersorte ausgegangen war, daß zwischendurch einzene Lagen mit anderem Papier bedruckt worden waren. Gene La men Genanso wuden dis Kupfor gleichen Anlaß, mehrmals aus lech blaulichem Papies gedruckt. Da sich gerade über die Qualität des Abdrucks der Kupfertafeln auf dem nicht ganz glatten gerippten Papier hin und wieder Beschwerden ergeben hatten, ba Dingler 1829 den Freihernn von Cotta, für dic Kupfe tafeln ganz weißes und oberflächenglattes, über "Velinformen « hergestelltes Maschinen-Druckpapier zu verwenden. Trotz der höheren Kosten bewilligte Baro Cotta sofort dice Unstllung bei den Kupfertafeln au das ungerippte, auf feinerem Kupferdrahtgewebe geschöpfte Velinpapier ${ }^{120}$.

Während des gesamten Untersuchungszeitraumes is das Polytechnische Journal in der Frakturschrift gedruck worden. Einzig ausländische Titel hat man, besonders in den Anfangsjahren, in den ansonst in Fraktur gesctzten Inhaltsverzeichnissen oder auch in den Ubersichten über die neueste polytechnische Literatur in Antiqua eingedie now fügt. Diese unterschiedliche Typen-Behandlung deutscher Es ist 1835 auch intern ein Probedruck in Antiqua
gemacht worden. Den beiden Dingler gefiel dieser Antigemacht worden. Den beiden Dingler gefiel dieser Antiqua-Probedruck schr gut, und Johann Gottfried Dingler empfahl Cotta, in dessen eigenem Interesse diese Schrift Eem Tre sen, daß das Polyte Schritsatz, das heibt ofne Geßen Duschischen den Zeilen gesetzt wurde. Der kompresse Druck
des Journals war zwar in einer Vorbemerkung den Le-

${ }_{110} \mathrm{CA} \quad \mathrm{T} / 254$ 5.12.x845; CA II/22 Ir.x2..8445; CA II $/ 3 \mathrm{x}$ Ix.7. 7852

${ }^{120} \mathrm{CA} / / \mathrm{I79}$ I9.I2.I829; CA I/I80 31.I2.I829 ${ }_{121} \mathrm{CAI} / 238$ I5.12.1835. ern im Io. Band $x 823$ als ein gemeinnütziges Unternehmen des Verlages angepriesen worden. Dieser geringe Durchschuß hatte aber schon 1825 den Baron von Cotta beim Lesen des Journals gestöt $t^{122}$. Bei der Änderung der Papiersorte, der Ausstattung und des Satzspiegels im Band 99 ist der Durchschuß größer geworden, und daraus resultiert im wesentlichen die oben bemerkte Verringeung der Zeilenzahl pro Kolumne. Dic Frakturschrif unde

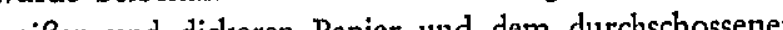
weißen und dick. atz ist jedenfalls das Satzbild des Polytechnischen Journals damals gefälliger und lesbarer geworden. Nach dem Wunsch der Herausgeber sollte bei dieser "Innovation des Polytechnischen Journals auch die frühere Orthographie der Allgemeinen Zeitung, welche bisher beibehalten worden war, "endlich" abgeschafft werden ${ }^{123}$

Anläßlich dieser "Innovation" des Aussehens des Journls Joun Toun Dingler und dessen sbishenge charakteristisch Einfassung der Vater Dingler belbehalten Wissen wollte $\mathrm{d}^{123}$. Ubber das Aussehen der Heftumschläge und übe die offensichtlich damals eingeführte Vignette auf dem Umschlag kann im einzelnen allerdings nichts mitgeteil wcrden, da diese beim Binden der Bände abgetrenn worden und daher verloren gegangen sind. Einzig bei Dietze in seiner Geschichte des Journals ist auf Seite 4

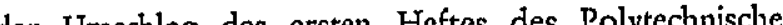
Journals abgebildet.

\subsubsection{Die Tafeln}

Der Text einer Zeitschrift, die zur technischen Schulun der Gewerbetreibenden beitragen und zum unmittelbaen pratiche pre mußte notwendig durch die Beigabe möglichst exakte und ins Detail gehender Zeichnungen ergänzt werden, "damit etwas sehr vollständiges und fürs Leben brauchbares geliefert würde «124. Die Tafeln des Polytechnischen Journals bilden daher einen wesentlichen und integricrenden Bestandteil der Zeitschrift.

Jeder Band des Journals war mit vereinzelt maximal

Je minima 5 Kupfer- oder Steindrucktafeln ausgesteter Die hö nen Tafeln und damit die größten Schwankungen in der

${ }^{222} \mathrm{CAI} / 89$ 8.Ir.I825.

${ }_{123} \mathrm{CA}$ II/22 II.T2..x845; CA II $/ 20$ x.x2.x845; CA II/23 3.x. $x 846$

${ }^{24} \mathrm{CA} \mathrm{I} / 6$ ×4.8.1819, Dietze: Geschichte, S.4. 
Tafelzahi wurden erreicht in den Anfangsjahren des Polytechnischen Journals bis 1828 . Nach den ersten I830er Jahren haben sich dic Beigaben bei 6 bis 7, gelegentlich 8 Tafeln stabilisiert. In den $x 850 e r$ Jahren überwiegen deutlich die Bände mit 6 Abbildungen.

Ähnlich wie beim Druck des Journals legte Johann Gottfried Dingler schon bei der Gründung der Zeitschrift großen Wert darauf, daß die Kupfertafeln in Augsburg unter seinen Augen und seiner Aufsicht gestochen würden. Cotta trat hingegen zunächst mit Erfolg für Stuttgart ein ${ }^{124}$. Da dic Zeichnungsvorlagen für die Kupfer und Lithographien von Dingler in Augsburg hergestellt wurden, wohin die fertigen Platten von Stuttgart wicder geschickt wurden, war dies eine sehr umständliche Regeunv Als Dingler berin $28 \mathrm{Ig}$ Coth utherzeugte, Kuperich Aulden zusätzlich der eigcns becchnetn Schrift koste, während man in Stuttgart dafur 22 Gulden verlange, hat der Verleger offensichtlich der Herstellung der Kupferstiche in Augsburg zugestimmt ${ }^{125}$. Die Lithographien wurden $\mathrm{zu}-$ nächst weiterhin in Stuttgart angefertigt und waren eine Quelle wiederhoiter Irrungen und Verzögerungen in der Fertigstellung des Journals ${ }^{120}$. Wegen der Verzögerung in der Fertigstellung des Journals bei den lithographiin schen Tafn Pai I82O daDotta nochmals vor, daß er jetzt nach langem Handeln die lithographischen Platten wic die Kupfer um I6 Gulden zuzüglich der Schriftenstiche von $2 \mathrm{fl} 2 \mathrm{kr}$ erhielte. Die Kupferstiche kämen damit insgesamt letztlich billiger als die Lithographien, und es sei bei diesen auch in der Art der Arbeit in keiner Weise irgendein Gewing ${ }^{127}$ Bei den Kupferplatten könne überdies noch etwns werden, da cr sich bemïhen worde, di Plaren gerent uffillen und keinen uufullen und keinen Raum ungenuitzt verloren gehen $z u$

Dingler hatte schon bei der Erlangung des Zugeständnisses von seiten Cottas zur Anfertigung der Kupfertafeln in Augsburg das wirtschaftliche Moment sehr stark in seiner Argumentation herausgestellt, indem er betonte, er hätte bei der ersten Kupferplatte "alle Oekonomie beobachten lassen und die vershiedenen $\mathrm{Oes}$ stände auf eine Platte rebracht olune Stöhung des fälligen «128. Dinger lam en des Verlogers anceren, den dabsichWiedes Wiedergabeverfahren mittels Kupferstich und Lithogra-

${ }^{128} \mathrm{CAI} / 8$ 28.9.x8I9

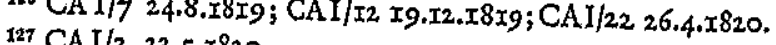

${ }_{128} \mathrm{CAI} / 9$ 20.x0.7819; CA I/x5 22. phie zu scharfer finanzieller Kalkulation und zu größtmöglicher Ausnutzung des zur Verfügung stehenden Plattenraumes zwang. Das lithographische Verfahren ist bei den Tafeln neben den Kupfern beibehalten worden, doch sind als Folge von Dinglers Einwänden entweder schon 1820 oder wenig später auch dic Lithographien neben den Kupfern in Augsburg hergestellt worden. Angefertigt wurden die Tafeln, soweit über die Namen der einzelnen Zeichner wie Aloys Dusch, Jos. Hutter, Wolff und G. Wengg hinausgehend Angaben gemach werden, zunächst von Gombart \& $\mathrm{Co}$. in Augsburg. A x836 wurden die Tafeln des Polytechnischen Journals zunächst noch gemeinsam mit Gombart, dann durch Jahrzehnte allein von der Lithographischen Ansult che In 1834 ware in Kuperstecher und ein Lithraph für das Journ beschäftigts « ${ }^{130} . \mathrm{Ob}$ allerdings beide ausschließlich für da Journal arbeiteten, ist unbekannt. Auf dem Titelblat des Polytechnischen Journals wurden bis zum Band 99 (I846) die Tafeln generell als "Kupfertafeln" bezeichnet erst ab Band Ioo trat dann an dessen Stelle die neutralere Bezeichnung "Abbildung".

Zur bereits angesprochenen optimalen Ausnutzung der Platten sind die Tafeln in der Regel randvoll mit Abbit dungen ausgefüllt. So sind laut Titelblatt im 6. Band (I82x) auf den 6 Kupfertafeln 38 Maschinen durch 2 Figuren abgebildet. Umfangreichere Konstruktionen, die mehrere detaillierte Abbildungen erforderlich machten, sind mit eigenen Überschriften auf den Tafeln versehen Dic Beschreibung der Funktionen und Wirkungsweise

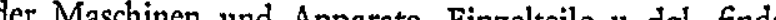
Dich und Kleinbuchstaben oder Ziffern im Artikeltext und auf den Tafeln ist der Zusammenhang zwischen Text und Abbildungen gewahrt. Allerdings ist nicht immer bei den Konstruktionszeichnungen der Maßstab angegeben. Dies war ohne Zweifel eine Folge der Abhängigkeit von den Vorlagen in den ausgewerteten Zcitschriften und bedeutete für das Nachbauen der Maschinen und Appante durch Gever schwernis.

Das Format der Tafeln ist recht unterschiedlich. E liegt in den späteren Bänden bei starken Schwankungen $\mathrm{m}$ einzelnen bei rund $50 \mathrm{~cm}$ in der Breite und etwa be $r 8-20 \mathrm{~cm}$ in der Höhe, wobei pro Band nahezu stets eine oder zwei, gelegentlich auch drei Tafeln als Aus-

${ }^{120}$ Siehe Z.B.: Polyt. Journal $2 \mathrm{I}$ (I826); 24 (I827); $5 \mathrm{I}$ (I834)

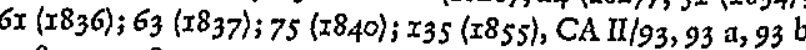

27.8. -9.9 .1873$.
$130 \mathrm{CAIII} / 525 . x \mathrm{xr} .1834$ nahmcfall 37 bis $40 \mathrm{~cm}$ hoch waren. Die Tafeln haben das Journalformat damit zum Teil erheblich überschritten und konnten bzw. mußten zur Benutzung aufgeklappt werden.

Die Tafeln wurden mit den einzelnen Heften ausceliefert, zu denen sie inhaltlich gehörten. Sie wurden dann beim Binden des jeweiligen Bandes entweder am Schluß des Bandes als eigener Tafelanhang zusammengestellt oder nach dem jeweiligen Einzelheft mitgebunden.

Neben den Tafeln waren als weitere Texterläuterungen im Polytechnischen Journal, allerdings nicht getrennt vom Text, sondern dem Textsatz eingefügt, noch Tabellen beigegeben. Es handelt sich hier meist um Zahlen irsendwelcher Untersuchungswerte in Spaltenform, um Berecher Une (n) bis zum Band 99 eigens auf dem Titelblatt des Journals aufgeführt.

Während des Berichtszeitraums vollzog sich bei den Illustrationsverfaliren als bedeutende Veränderung der Wiederaufstieg des besonders durch den Engländer Thomas Bewick zum Holzstich verbesscrten alten Holzschnittverforens. Erstmals ist Ende 1845 in den Briefen Dingen an Cotn die Rede vom Eingty des Holzschnitso zur Illustration des Polytechnischen Journals. Emil Maximilian Dingler schrieb damals an Baron Georg von Cotta: "In der Folge werde ich nicht ermangeln bei $\mathrm{Ab}$ bildungen, welche sich für Holzschnitt eignen und dem Text beigedruckt werden können, Ihrem Wunsch zu entsprechen. «131 Es wurden nun tatsächlich Holzschnitte in Stuttgart sowie auch in München für das Polytechnische Journal angefertigt. Die Vorlagen wurden besonders lisch Zeitoliften a

Wegen der Schwierigkeit in der Darstellung feiner und Weger dinien beim Holzschnitt war Emil Dingler mit dem Verfahren keineswegs generell zufrieden. In einem undatierten Schreiben, das von Anfang I846 stammen muß, faßte er seine Grundsätze bezüglich des $\mathrm{Holz}_{\text {- }}$ schnitts zusammen. Hinsichtlich der Holzschnitte sei er bisher dem Grundsatz gefolgt, welchen die englischen und französischen wissenschaftlichen und technischen Zeitchifen beober ien nur solche Zcicten beobach irt sind, worin keine große Genauigkeit in den Dimensionen der Details beybehalten werden muß, kurz, welche mehr Ansichten der Gegenstände zur Verständigung derselben darzustellen haben \& deren Beschreibung

${ }^{101} \mathrm{CA}$ II/ $/ \mathrm{xg}$ x8.Ix.I845.

(1) $5.72 .7845 ; \mathrm{CA} \mathrm{II/20}$ r.I2.I845; CA II/23 3.r.I846 den Raum einer Columne nicht ïberschreitet, so daß der Leser der Abbildung die Beschreibung stets gegenübe hat! « Die englischen Lehrbücher der Physik, Chemie und Mechanik enthielten wie die bei Vieweg erschienenen Lehrbücher ausschließlich Holzschnitte, da die Abbildunzustellen hätten. Dagegen enthielten die guten englischen technischen und wissenschaftlichen Zeitschriften nur wonige Holzschnitte, da der Holzschnitt für kompliziertc Abbildungen in klcinem Maßstab nicht geeignet sei. Die Zeitschriftenartikel seien auch zu lang, "sodaß dem Lese die Vergleichung mit der Figur ungemein erschwert würde, während er die Abbildung auf einer herausgeschlagenen lithographischen Tafel stets vor Augen behält. Endlich kommt die Ausführung einer so große Anzhl kicher schen Platte vereinigt werden kônnen, in Holzschnitte unverhaltismalig hoch zu stchen, wahrend bey denselben die Schärfe \& Reinheit der lithogr. Zeichnung doch nic erreicht wird." Er meinte auch noch, daß mit den rohen Holzschnitten in der Art, wie sie eine populärtechnische englische Zeitschrift bringe, sich das deutsche technische Publikum nicht begnügen wärde $e^{13}$.

Das Polytechnische Journal hat trotz dieser Bedenken Dinglers auch später Holzschnitte gebracht. Wie dio Konkurrenz "Polytechnisches Centralblatt《 hat man aber nur sehr einfache, leicht ausführbare Figuren in Holzschnitten anfertigen lassen, welche man laut einer Anzeige in der "Allgemeinen Zeitung" von 5846 in den Text zu drucken gedachte ${ }^{134}$.

\subsubsection{Das Register}

Bei der Fülle und Breite der im Polytechnischen Journal angeschnittenen Themen und der raschen Erscheinungsfolge der Hefte und der Bände war eine InhaltserschlieBung durch ein Register unumgänglich notwendig. Durch eine derartige Möglichkeit des Wiederauffindens und Nachschlagens von Artikeln wurde der Anwendungswert des Blattes für die Praxis erheblich crhöht. Dieser Tatsache eingedenk, hat Johann Gottfried Dingler von Anfang an dem letzten Band des jeweiligen Jahrgangs ein Namen- und Sachregister für die 3 brw 4 Bände des abgcschlossenen Jahrgangs beigcgeben. Dieses Register fuihtte in alphabetischer Ineinanderordnung von Namen und Sacen alle in den Titeln vorkommenden Personennamen und kennzeichnenden Sachstichwörter auf. Von

${ }^{133} \mathrm{CA}$ II $/ 99$ undatiert (I846).

${ }^{204} \mathrm{CA}$ II/24 23.rr..5847; CA II/65 5.6.5863; CA II/99 undatiert (1846). 
größtem Wert für die Benutzung war dabei, daß man sich bei den Stichwörtern nicht auf das wesentlichste oder kennzeichnendste Wort beschränkte. Man nahm vielmelar auch alle anderen im Aufsatztitel vorkommenden Hauptrichörter bei der Registerherstellung aut Diese engmaschige Aufschlüsselung nach den unterschicdlichsten Stichwörtern erleichterte das Wiederauffinden eines Titels oder eines Verfassers. Durch Verweisungen wurden Parallelstellen aufgezeigt. Aufsätze aus dem Beitragsteil und Miszellenanmerkungen wurden gleich behandelt. Bei den oft seitenlangen Zusammenstellungen englischer Patente in den Miszellen wurden die Namen der Patentinhaber in das Register aufgenommen. Sachlich wurden diese $\mathrm{P}$ ater men. Sach hurden diese Pante in $c$ menstellungen nicht mehr erschlossen.

Die Register sind in.Spalten angeordnet. Bei den Stichwörtern selbst wurde das auszuwerfende Wort nach links ausgerückt und rechts daneben der Gesamttitel oder ein Teil des Titels nochmals wiederholt. Zwci Beispiele sollen das Verfahren demonstrieren:

Der Artikel "Brunels neueste Versuche über die Anwendung des hydraulischen Kalks bein Brükenbaue olne daß man dazu Bogengerüste nötig hatu in S. 305 ft wind im daugchörigen S. 305 ff. wird im dazugehörigen Register, Band 50 folgendermaßen aufgesclliüsselt

Brunel, Versuche über die Anwendung des hydraulischen Kalks beim Brükenbaue XLVII, 305 .

Brüke, Anwendung des hydraulischen Kalks dabei nach Brunel XLVII,305.

Kalk, Anwendung des hydraulischen Kalks beim Brükenbau nach Brunel XLVII,305.

Der Artikel "Ueber eine Vorbereitung der Kolks, um in den Hohöfen schwefelfreies Roheisen zu erzeugen; von Profcssor Calvert in Manchester" in Band I26, S. IX2 ff. wird im Register desselben Bandes folgender-

Calvert, über Entschwefelung der Kohks beim Hohofenbetrieb CXXVI.rIx.

Eisen, Calverts Verfahren schwefelfreies Roheisen mit Kohks zu erzeugen CXXVI.Ix 2

Roheisen, siehe Eisen.

Kolks, siehe Steinkohlen.

Steinkohllen, Verfahren schwefelfreie Kohks zu exzeugen,

von Calvert und Barthelemy CXXVI.rI2.294.

Das Jahresregister wurde von der Redaktion erst "nach Fertigung des ganzen Jahrgangs ... ausgezogen damit es gleichsam aus einem Guß hervorgeht « ${ }^{136}$.

${ }^{195} \mathrm{CAl} / 2545.52 .1845$
Mit der steigenden Bändezahl verminderte sich der Wert der Jahresregister, da zu viele Registerbände zum Wiederauffinden eines Artikels durchgesehen werden mußten. Es erschien daher erstmals $I 843$ ein die Bände I-78 zusammenfassender Real-Index als Generalregister Ium Polytechnischen Jour Reister ohn Bericksichtigung der Autorennmen. Die Rubrizierung erfolgte auch jetzt unter einem Stichwort, das nach links ausgerückt und zur besseren Ubersicht fett gedruckt war. In gewissen Fällen besaßen diese Stichwörter durchaus Schlagwortcharakter, weswegen offensichtlich zum lcichteren Wiederauffinden von Artikeln dem Realregister später noch ein Wort-Register angefügt ie Bände $x-78$ in vier Abschnitte aufgeteilt, die jeweils wieder im Alphabet von vorne beginnen.

Der Real-Index ist unter der Anteilnahme, aber olne eigentliche Mitarbeit von seiten der Herausgeber erstellt worden ${ }^{130}$. Dingler hat zwar bereits 1828 an Cotta gcschrieben, daß über die ersten 30 Bände der Anfang zum Realregister gemacht worden sei ${ }^{137}$. Es hat aber dann noch Is Jahre gedauert bis die Bände I-78 im General. register vorlagen und zwar erstellt von Dr. Michael Stecker, k.k. Universitäts-Professor und Sekretär der Landwirtschafts-Gesellschaft in Wien. Vater und Sohn Dingler fanden das Register "in bezug auf Rubrizierung der Gegenstände überhaupt in der ganzen Anordnung selhr zweckmäßig « ${ }^{138}$. I853 folgte in der gleichen Anordnungsweise mit rund 20 Bände zusammenfassenden Unterabteilungen, Wortuegisten und fettediucken stellungen stellungen der Real-Index für die Bände 79 bis II8. x86I wurden noch die Bände $x \times 9$ bis $x 58$ in einem 3 . Band durch das Generalregister erfaßt. Verfasser dicser beiden Registerbände war anstelle von Prof. Stecker D. Philipp, Bibliothekar der polytechnischen Gesellschaft in Berlin, der sich für diese Arbeit den Herausgebern und dem Verlag selbst angeboten hatte ${ }^{130}$. Die Registerbände sind insgesamt ein herrorragendes Ausk ftsmitel iber die Viffer der in Polyechendes die Vielfalt der im Polytechnischen Journal behandelten 3.5 Der Aufbau des Journals 3.5.I Die Beiträge

Der bedeutendste und inhaltlich wichtigste Teil des Polytechnischen Journals wird von umfangreicheren Beiträ-

${ }^{130} \mathrm{CA} \mathrm{II} / 28$ I8.8.x85I; CA II/49 x.2.x860.

${ }^{137} \mathrm{CAI} / \mathrm{x} 72$ 30.x0.1828.

${ }^{108} \mathrm{CA} \mathrm{I} / 2.48$ 16.2.1842.

${ }^{130} \mathrm{CA} \mathrm{II/28}$ I8.8.x85I; CA II/30 x.I2.I85I. gen eingenommen. Es handelt sich dabei um Artikelübernahmen aus meist ausländischen Zeitschriften in ollständiger, frei übersetzter, umgearbeiteter oder gokürzter Form und um Originalaufsätze.

Alle Artikel aus nichtdeutschsprachigen Zeitschriften wurden übersetzt. Vereinzelt wurden auch Auszüge aus Monographien übernommen. Jeder Aufsatz enthält im oder unter dem Titel den Verfassernamen und Namen, Jahrgang, Band oder Nummer der ausgewerteten Zeitschrift. Sachkundige Anmerkungen in Fußnoten, Erläuterungen oder weiterführende Hinweise, Einwendungen Tegen durgelegre Ausführungen oder Bedenken gegen Cffich Serter zu mehreren Seiten am Schluß eines Artikels seitens der
Herausgeber oder der Obersetzer gehören mit zum norHerausgeber oder der Ubersetzer gehören
malen Erscheinungsbild des Beitragsteils.

malen Erscheinungsbild des Beitragsteils.

Dem Charakter der Zeitschrift als Referatergan zwe weitere polytechnische Kreise ansprechen wollte, sind dic Artikel gemeinverständlich abgefaßt. Durch oft »freic Obersente arauf sichtlich Wert. Es finden sich allerdings imme wieder Artikel mit mathematischen Berechnungen und Formeln, die Kenntnisse in der Mathematik verlangten wie sie von einem einfachen Gewerbsmann schwerlich erwartet werden durften. $D_{a}$ diese Artikcl eher gege Ende des Berichtszeitraums auftreten, hat man dari einc zeitkonforme ingenieurmäßigere Steigerung des $\mathrm{N}$ veaus des Polytechnischen Journals zu sehen ${ }^{140}$.

Die Anordnung der Beiträge erfolgte nicht systematisch nach bostimmten Themenbereichen. Bei dem damaligen Entwicklungsstand der Technik als Disziplin und angesichts der ersten Versuche der technischen Berichterstattung war eine systematische Anordnung billigerweise auch nicht $\mathrm{zu}$ erwarten. Vielmehr stehen dis Artikel verschiedensten Inhalts unverbunden nebeneinander, wie sich dies zufällig aufgrund der Zeitschriftenauswertungen ergab. Die Redaktion hat sich aber nich selten beïh anfallende Artikel mit einem gleiche celych oder ahnnlichen Them in in Journal hintereinander in einer Folge abzuch fahren war aber mehr Ergebnis eines glücklichen Zusam mentreffens gleichthematischer Artikel in der Zeitschriftenauswertung als zielbewußte oder klare redaktionelle Konzeption. Immerhin hat das $x_{5}$ Jahre später als da Polytechnische Journal entstandene Polytechnische Centralblatt von Julius A. Hülsse »bei gedrängter aber doc

${ }_{140}$ Siehe z.B. Polyt. Journal Band 78, 8I, II2, II4, I20, I25. vollständiger Darstellung durch Gruppirung verwandter Gegenstände so viel möglich die Uebersicht erleichtert" ${ }^{141}$.

Die Zahl der Beiträge pro Band schwankt erheblich. Sie ist von 52 bzw. $5 x$ Beiträgen im ersten und zweiten im 2x. Band (1826) angestiegen. Es ergibt sich also in den Minima und Maxima eine Differenz von nicht weniger als 96 Beiträgen. In den Jahren nach 1826 lag die Zahl der Beiträge mit vereinzelten Ausschlägen mach unten und nach oben in der Mehrzahl der Bände zwischen IIO und I3O Attikeln. Mitte der I830er Jahre sank die Beitragszahl ab bis auf Werte zwischen 72 und TO5. Seit dem Ende der I83oer Jahre bis zum Berichts-

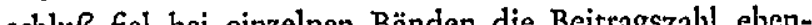
schl $B$ fel be einzen Banden die Beitagsenfalls unter 100, in der Mehnheit der Bando enelhelt aber das Journal zwischen 100 und $x 20$ Beiträge. Gelegentlich wurde auch die Zahl von I20 Beiträgen überschritten, aber die Marge von $x_{30}$ nicht mehr erreicht. Dabei ist zu sagen, daß ab Band 99 ( 2846$\rangle$ die Miszellen nicht mehr bei den Beiträgen mitgezählt wurden, so daß ab dicsem Band gegenüber früheren Bänden die Zahl der Beiträge automatisch sich um sechs verringerte.

\subsubsection{Die Miszellen}

Die Miszellen umfassen Kurzbeiträge und Notizen vermischten Inhalts zu den verschiedenartigsten Themen. Sie stehen jeweils nach den Aufsätzen am Schluß eines jeden Heftes. Jeder Band umfaßt demnach bis zum Beginn des Jahres r826 vier und später sechs Miszellen.

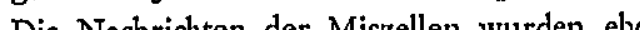
wiegend ausländischen Zeitschriften entnommen. Der Zweck der Miszellen war, in ihnen neben technischen und naturwissenschaftlichen Artikeln stärker als im Beitragsteil zur Unterrichtung der Leser auch handelskundliche, betriebs- und volkswirtschaftliche Probleme sowie verkehrs- und wirtschaftspolitische Fragen aufgreifen $\mathrm{zu}$ können. Bei einer ganzen Reihe von Miszellen-Artikeln ist es aber so, daß $\beta$ sie genauso gut im Beitragsteil hätten unterce tenzaben der Miszellen haben von Heft zu Heft erheblich differiert:

$\operatorname{Im} x$. Band von $x$ bis $x x$ Seiten, im 2x. Band von 7 bis ${ }_{24}$ Seiten, im 42. Band von 7,5 bis 20 Seiten, im 62. Band von knapp 6 bis $x_{4}$ Seiten, im 79. Band von 5,5 bis knapp 26 Seiten, im 9r. Band von 4 bis 9 Seiten, im to5. Band von 7,5 bis Ix,5 Seiten, im $I 30$. Band von 6,5 bis 8,5 Seiten und im Band $x_{3} 8$ von knapp 6 bis $x_{2}$ Seiten.

${ }^{141}$ Karmarsch: Geschichte, S. 894. 
In den späteren Jahrcn lag im Durchschnitt die Seitenzahl der Miszellen pro Heft unter xo Seiten. Etwa ein Zehntel, manchmal auch etwas mehr der Seiten pro Heft entfiel auf den Miszellenteil. Extrem große Schwankugen im Umfang der einzclnen Miszellen, wie diese $Z$. bei Band

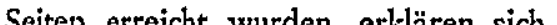

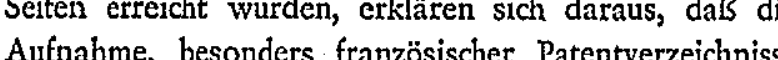

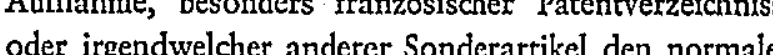
oder irgendwelcher anderer Sonderartikel den normalen Rahmen sprengten.

\subsubsection{Polytechnischer Anzeiger}

Johann Gottfried Dingler war trotz seines eigenen unternehmerischen Mißerfolgs eine Person mit einem ausge prägten Gespür für zukunftsträchtige Entwicklungen und Ideen. Seit den letzten Jahrzehnten des I8. Jahrhundert hatten bereits vereinzelt Zeitschriften Geschäftsanzeige aufgenommen. Die Beigabe eines Anzeigenteils bei eine Zeitschrift war aber zu Beginn des Ig. Jahrhunderts noch keineswess solbstrerstandlich. Dingler sh jodoch berts

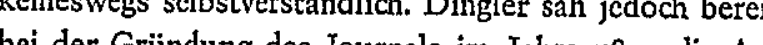
bei der Grundung nahme von bezahlten Anzeigen als wesentlichen Beitra zur Abdeckung der Herstellungskosten an, zumindest sollten diese, "wenn auch nicht ganz, doch einen Theil des Honorars decken ${ }^{142}$. Ebenso wollte Dingler, wie bcreits dargelegt, bei seinen Zeitungsplänen stets einen Teil der Unkosten durch Avertissements bzw. ein durch Inserate finanziertes Intelligenzblatt decken.

Die Beigabe eines Polytechnischen Anzeigers zum Polytechnischen Journal war also sicherlich in erster Linio der Initiative Johann Gottfried Dinglers zu verdanken In diesem Anzeiger sollten »Anzeigen jeder Art schnell aufgenommen" werden, das heißt, sic sollten in den ersten nach Empfang der Einsendung crscheinenden Hefte angezeigt werden. Nach einer Redaktionsangabe aus dem Jahre 1834 sollte die Zeile mit 6 Kreuzer be rechnet werden. Gedacht war dieser Anzeiger nach de Worten des Herausgebers fiir Bekan Ane rischer und anderer Gegenständc, dann für Anstellungs gesuche, Verkaufsgegenstände etc. Korrespondenzstelle für Anfragen und Annahmestelle sollte die Expeditio der Verlagshandlung sein ${ }^{143}$. Der Polytechnische Anzeger stand jeweils am Schluß der Hefte.

Dieser meist nur zwei oder wenig mehr Seiten umfassende Polytechnische Anzeiger hat nicht die Bedeutung

${ }^{242}$ CA I/5 8.7.r8x9; Schulz: Pachpresse, S. $46 \mathrm{f}$; Dietzo Geschichte, S. 3 . ${ }^{133}$ Polyt. Journal 30 (I828), S. IV; Börscnblatt I (x834)
Sp. 273 .

ler bei Cotta urgicren, ner möchte einige Avertisse ments ins Journal drucken lassen, um den Anzeiger zur Welt zu bringen ${ }^{144}$. Der erste Band des Journals weis denn auch keinen Polytechnischen Anzeiger auf. Es gib zwar vereinzelt Bände, in denen jedem Heft ein Anzcige beigegeben war, z.B. Band 6. Es gibt aber vicle Bänd mit nur einem einzigen oder vielc Bände mit überhaup keinem Anzeiger.

In den ersten Bänden finden sich auch einige echte Geschäftsanzcigen im Anzeiger, in denen zum Beispiel Fabriken zum Kauf angeboten wurden. Ansonsten haben ber vornehmlich Verlage den Anzeiger zur Werbung fü ihre technischen und naturwissenschaftlichen Neuerscheinungen genutzt. Es haben in ihm unter anderem, in eine zum Teil den Inhait der Neuerscheinungen referiexenden oder rezensierenden Art inseriert die Verlage Hemmerde \& Schwetschke in Halle, Joh. Ambr. Barth in Leipzig Duncker \& Humblot in Berlin, J. D. Sauerlander in lung in Augsburg, die Keyser'sche Buchhandlung in BrEichstätt. Vor allem hat aber dic Cotta'sche Buchhandlung selbst im Polytechnischen Anzeiger ihre neue polytechnische Literatur angezeigt ${ }^{145}$. $\mathrm{Da}$ aber die Redaktion in den Miszellen stets die neueste in- und ausländische polytechnische Literatur anzeigte ind zum Teil terensierte, hat sie Verlagsinserenten im Polytechn zeiger den Ansporn zum Anzeigen der Werke genommen.

Für den Bereich aber, der später das technische Anzeigengeschäft trug, nämlich die Vertricbsförderung un Werbung technischer Artikel und Maschinen mittels der Annonce, war die Zeit noch nicht reif. Die mechanischen noch vorwiegend von der Befriedigung der Nachfrage eincs lokalen oder regional eng begrenzten Marktes und waren als Industriezweige auch noch kaum entwickelt. Erst als Deutschland durch den Zollvereinheitlichung zu cinem einheitlichen weitrüumigen und anonymen Markt zusammenwuchs und besonders der Maschinenbau ein eigenständiger Industriezweig wurde, gewannen um die Mitte des tung. Insofern war der von J. G. Dingler konzipierte

144 CAI/I6 17,2.1820 146 Einzelne Nachweise siche z.B. Polyt. Journal Band 2, 4, 6 ,
7, 9, IO, I2, I3, I4.
Polytechnische Anzeiger ein verunglücktes Vorhaben, als er seiner Zeit voraus war ${ }^{110}$. Noch 1834 erweckte de Verlag Cotta in einem Werbetext zun Polytechnischen Journal den Eindruck, als ob vom Jahre I834 an erstmols das Journal mit einem Polytochnischen Anzerter furt, die Verlage J. G. Calve in Prag und J. M. Bayer in Rabrikationszweige der Frïhindustrinlisen lobren derts die technischen Anzeiren
4.I Ausgewertete Zeitschriften ${ }^{140}$

4.x.x Englische Zeitschriften

Wie bereits ausgeführt, hat vor allem Englands entwickelte Industrie und technische Berichterstattung die Anregung und den Nachrichtenstoff für die deutsche technische Journalistik in deren Anfangsstadien geliefert. Hier in England sind in den regelmäßigen Veröffentlichungen der Beschreibungen der schon I 623 durch Statu geregelten Patentgenehmigungen überhaupt die Wurzel der technischen periodischen Berichterstattung zu suchen. Aus dieser Patentliteratur schöpften bis weit in das $x g$. Jahrundert hinein die Journale aller Länder einen groJah Teil ihes techich Polytechnische Journal macht hierin keine Ausnahme. Es stehen bei diesem nach der Zahl der aus fremden Zeitschriften übernommenen Beiträge weitaus diejenigen englischen Journalc an der Spitze, welche dic Beschreibungen der einzelnen englischen Patenterteilungen und Patentübersichten veröffentlichten. Das war bereits ab Beginn des Journals das Repertory of Arts, Manufactures and Agriculture, Second Series, das seit I794 in Lon Intions ur Lurcerions unber stammen in carten Band des Polytechischen Jounds 16 von insgesamt 26 englischen Beiträgen. Im 5. Band sind

${ }_{140}$ Schulz: Fachpresse, S. 47 ff. ${ }^{247}$ Börsenblatt $x(x 834), S p .273$ ${ }^{188} \mathrm{CAII} / 3 \mathrm{I}$ I.7. .8852

${ }^{240}$ Zur Methodik: Es wurde jeder тo. Band sowie Band 5 und des Polyt. Journals daraufhin untersucht, aus welchen ZeitDie Grundlinie der Zcitschriftenauswertung charakterisicren zu können.

Für die kurzen bibliographischen Angaben und dic häufig notwendigen Titelergänzungen bei den Zeitschriften wurden die einschlägigen französischen, englischen, deutschen und merikanischen Zeitschritenkataloge herangezogen.

${ }^{300}$ Schulz: Fachpresse, S. 9f; Karmarsch: Geschichte, S. I43

ger hat trotz dieser Anzeige auch in diesen Jahren keinen entscheidenden Aufschwung erfahren. Hart an der Schwelle des eigentlichen Bedeutungsanstieges des technischen Annoncengeschäfts, nämlich $x 852$, wurde offiziell im »Ausland" dem Publikum das Erlöschen des Polytechnischen Anzeigers mitgeteilt ${ }^{148}$.

\section{Die Herkunft des Nachrichtenstoffes}

es 33 von 45 englischen Artikeln, welche ihm entnommen wurden. Nachdem seit dem Jahre I82O als zusätzliches Berichtsorgan der neuerteilten englischen Patente das London Journal of Arts and Sciences herausgekommen war, ist im ro. Band des Polytechnischen Journals 2 mal das Repertory of Arts usw. und $x 6$ mal das London Journal of Arts and Sciences bei insgesamt 66 englischen Artikeln ausgewertet worden.

Bis zum Ende des Berichtszeitraumes wurden nun da Repertory of Arts, Manufactures and Agriculture, Second Series bzw. das Repertory of Patent-Inventions sowie das London Journal of Arts and Sciences auf ihre rishe Patente hin ausgewertet. Auf diese beiden Zcitschiften und damit auf das Nachdrucken der englischen Patente entfällt die Hauptmasse der übersetzten englischen Artikel im Polytechnischen Journal. Während der gesamten Zeit $1820-1855$ waren meist erheblich mehr als $50 \%$ und nur singulär unter $50 \%$ aller in einem Band des Polytechnischen Journals übernommenen eng lischen Artikel Beschreibungen englischer Erfindungspatente ${ }^{161}$.

Seit $x 823$ bis 1858 erschien in London das Mechanics' Mngazine. Aus ihm hat Dingler nicht selten pro Band zwischen to und 20 Beiträge entnommen, mit allerdings rasch sinkender Tendenz in den I85oer Jahren ${ }^{162}$. Gemeinsam mit den zwei erstgenannten Zeitschriften gehö:t das Mechanics' Magazine auf Dauer zu den am häufigsten ausgewerteten englischen Zeitschriften im Polytechnischen Journal. Hinter ihnen bleiben alle anderen englischen Periodika zum Teil erheblich zurück.

${ }^{261}$ Dem Repertory of Patent-Inventions und London Journa of Arts and Sciences sind folgende Beiträge entlehnt worden In Band 2O: 55 Bciträge; Bd 40:22; Bd 50:32; Bd 60: $35 ; \mathrm{Bd} 7 \mathrm{O}^{\circ}$ 39; Bd So: 34; Bd 90: 25: Bd xoo: 28; Bd xx०: x9; Bd I20: $x 7$

Bd I30: $19 ; \mathrm{Bd} \times 38: x_{3}$.
${ }_{183}$ Aus dem Mechanics Magazine wurden entnommen: $\mathrm{Bd}$ 20: 77 Beiträge; Bd 30: 7; Bd $40:$ I4; Bd 50: I7; Bd 60: 9; Bd 70: I2; Bd 80: xо; Bd 90: x6; Bd I00: Ix; Bd Ix०: 8; Bd I30: 2 . 
Eine Reihe anderer englischer Zeitschriften, die nach ihrem Inhalt eine gute Nachrichtenquelle für das Polytechnische Journal hätten abgeben können, waren zu kurzlebig, um über einen längeren Zeitraum hinweg in stehen zu können. So ist das mit Abbildungen versehen Technical Repository von T. Gill (London I822 ff.), I827 als Gill's Technological Repository firmierend während seiner kurzen Erscheinungszeit in Band Io und Is je I8 mal und in Band 20 und 30 des Polytechnischen Journals je Ir mal ausgewertet worden. Das Register of Axts, mit seiner Fortsetzung $x 824-1832$ in London erschienen, ist in Band 30 des Polytechnischen Journ

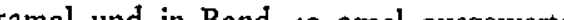
 in den Jahren $1824-1826$ brachte, ist in Band 20 neunmal ausgewertet worden. Gegen Ende des Berichtszei raumes stieg in den $5850 e r$ Jahren anstelle des Mechanics' Magazine das Practical Mechanics' Journal, sei I 848 unter diesem Titel exscheinend, als neue Spitzenzeitschrift unter den ausgewerteten englischen Zeitschritten auf. Ihm entstammen in Band $x_{20} x_{3}$, in Band $I_{30}$ Ix und in Band $x_{3} 8$ neun Beiträge.

Nach seinem Ersterscheinen in London im Jahre 1837 ist mit einigen Beiträgen pro Band in der Regel auch do Civil Engineer and Architects' Journal im Polytechnschen Journal vertreten, ebenso auch die Chemical Gazette (London $1842-1859)^{163}$.

Sehr beständig scheinen mit einigen Beiträgen im Polytechnischen Journal auch einige englische Zeitschriften auf, welche in ihrem Titel das Wort "philosophy oder "philosophical « führten, die aber in ihren Untercheln meist an erkennen Chem nagazine fut Cewerbsteb un del Gewerbsleben u. dgl. waren. Dies gilt am Anfang besonders für die Annals of Philosophy von R. Thomson, inschließlich neuer Serie $18 \times 3-1826$ in London erschienen, und das Philosophical Magazine and Journal by A. Tilloch and R. Taylor (London I8 $x_{4}-I 826$ ), die beido zuletzt vereinigt unter dem Titel London and Edinbure Philosophical Magazine and Journal of Science forme führt wurden ${ }^{154}$. Fügt man dazu noch das Edinburgh

${ }^{135}$ Dem Civil Engineer and Architect's Journal entstammen in Band $70,80,90$ je 2 Beiträge; $B d$ $x x 0: x ; B d x 20: x ; B d x 30: 6$ $B d I_{38}: 4$. Dic Chemical Gazettc ist in $B d$ I20, $x_{30}, x_{3} 8$ je $x$ ausgewertet worden.
${ }_{26 \pi}$ Die Annals of $P$.

Die Annals of Philosophy sind in Band I und 5 mit je und in Band xo mit 2 Beiträgen vertreten. Das Philosophice Magazin wurde in den gleichen Bänden 2, 4 und 2mal ausge-

Philosophical Journal von Brewster und Jameson (Edinburgh I8Ig ff.) und das als Fortsetzung davo r826 erschienene Edinburgh New Philosophical Jourma von $R$. Jameson hinzu und erwähnt man, $d a B$ in de ersten Bänden des Polytechnischen Jourmals besonders auch das Repository of Arts, Literature, Commerce, Manufactures, Fashions and Politics (London $1809 \mathrm{ff}$ ) ausgewertet wurde, so hat man die wichtigsten im Polytechnischen Journal ausgewerteten englischen Zeitschriten genannt.

Auffallend dabei ist, daß die Transactions of the Society for the encouragement of Arts (angefanen Vorbild zahlreicher ähnlicher Einrichtungen au dem Kontinent, fehlen. Sie lieferten zwar später nur mehr weniger "Gutes «155, sie sind aber im Polytechnischen Journal insofern wiederholt indirekt erfaßt worden (z. B. Band s), als Artikel aus ihnen über den Umweg der durch Dingler ausgewerteten Zeitschriften in das Journal gelangt sind. Dics ist eine Folge davon, daß ein Großteil der von Dingler ausgewerteten Zeitschriften Artikelïbernohmen in sehr viel größerer Krcis von Zeitschriften erfaßt worden als der, welcher tatsächlich ausgewertet wurde. Der gegen Plagiate allergische J. G. Dingler gibt denn auch stets an, aus welcher Zeitschrift die von ihm referierten Aufsätze ihrerseits ursprünglich ubernommen worden waren. Er erweckt allerdings gerade in den Anfangsbänden durch die Art der Zitienung manchmal den Eind ob er melr Zeitschriften in Oas ausgewertet hätte, als dies tächlich der Fall war. Der Ersterscheinungsort eines Beitrages steht nämlich gelegentlich als Zitatstelle unter dem Titel. Erst bei der Lektüre des Beitrages wird dann aufgrund eine Anmerkung, einer Notiz oder einer zusätzlichen Angabe ersichtlich, daß der Aufsatz tatsächlich dem engeren Kreis der von Dingler ausgewerteten Zeitschriften entnommen worden ist $t^{150}$.

Es ist nicht mehr Aufgabe dieser Arbeit, die Nachrichtenbeschaffung von Dinglers Nachrichtenguellen zu untersuchen, aber vermutlich ließen sich dabei regelrechte Wanderungen von technisch-naturwissenschaftlichen $\mathrm{Be}$ richten durch die zeitgenössische technische Zeitschriftenliteratur feststellen. Beiträge aus regionalen Gewerbeblättern oder speziellen Fachorganen, z. B. den in den ersten Jahrgängen häufig indirekt erfoßten Verhandlunen der Kaledonischen und der Londone Garen-Kulure Gesellschaften, haben auf dem Umweg über größere

${ }^{165}$ Karmarsch: Geschichte, S. 899. Die Transactions.. lieferten früher manches Gute

${ }^{200}$ Siehe z.B. Polyt. Journal 2 (I820), S. I, 4I.
Referateblätter den Weg in die internationale polytechnische Literatur gefunden. In Dinglers Polytechnischem Journal 1 iß sich durch $d$ Zweit- und Drittübernahmen auch schr deutlich feststellen, daß z.B. Nachrichten aus amerikanischen Zeitschriften über den Umweg der englischen Zeitschriften schon sehr viel früher den Weg in die deutsche polytechnische Literatur gefunden haben, noch ehe z.B. Dingler daran ging, amcrikanische Zeitschriften direkt $\mathrm{zu}$ abonnieren und auszuwerten. Man hat bei den Dingler'schen Herkunftsangaben auch den Eindruck, daß eine ganze Reihe urspriin rerung in englischen Zeitschriften das internationale Publikum erreicht haben. Der Anteil ursprünglich französischsprachiger Artikel lag daher in den Anfangsjahren des Polytechnischen Journals höher, als aufgrund der Herkunftsangaben in den Referaten sichtbar wird ${ }^{157}$. In gleicher Weise haben einige deutsche Autoren immer wieder ihre Aufsätze in englischen Zeitschriften veröffentlicht. Angesichts der Bedeutung der Patentliteratur

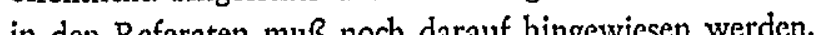
werden, daß um I820 in England "beinahe so vicle Patente auf Erfindungen von Ausländern als von eingeborenen Engländern crtheilt« wurden $1^{158}$, die damit ihren Erfindungen den guten englischen Patentschutz zuteil werden lassen wollten. Es sind damit keineswegs alle aus englischen Zeitschriften übernommenen Artikel ursprünglich originär englischer Herkunft gewesen. In dem bis zum Ende der r84oer Jahre andauernden, zum Teil erdrückenden Uberewicht der Artikelübernahmen aus englischen Zeit schriften im Polyto doch insgesamt das Ubergewicht der englischen Industrie und der technischen Berichterstattung bis zum Ausgang
der Frühindustrialisierung nieder ${ }^{250}$.

\section{T.2 Französische Zeitschriften}

Dic meist ausgewertete französische Zeitschrift über den gesamten Berichtszeitraum hinweg war das Bulletin de la Société d'encouragement pour l'industrie nationale ${ }^{100}$. Seit 1802 erscheinend, war es das Organ des I80I entstandenen, im Titel der Zeitschrift genannten Vereins,

${ }_{187}$ Z.B. Polyt. Journal 5 (I82I), xo (I823), $x_{5}$ (I824) ${ }^{288}$ Polyt. Journal 20 (x826), S. 83 f.

${ }^{100}$ Siehe Tafel I.

${ }^{200}$ Aus dem Bulletin de la Societé d'encouragement pour ionale wurden entnommen in Band $x: 2$ Bcitrage Bd 5 : $x$; Bd IO: 9; Bd xs: xI; Bd zo: Io; Bd 30: 5 ; Bd 50: 8 Bd 60: $x 3$; Bd 70: 8 ; Bd 80: 8 ; Bd 90: 4 ; Bd 100: 7 ; Bd Ix०: 8

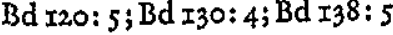

der nach dem älteren englischen Vorbild Einfluß auf dic Unterstützung und Hebung der nationalen Industrie ausäben sollte. Aus diesem Bulletin sind zum Teil die Uberichten ïber dio tramzösischen Patentgenehmigungen, in Patente iubernommen worden, ferner Ber ausschrciben, neue Verfahren und alle die vielfältigen Themen, durch welche diese staatliche und halbstaatlich Gewerbevereinsliteratur zur Schulung, Ermunterung und zum gegenseitigen Erfahrungsaustausch der Gewerbetreibenden beizutragen hoffte. Aus den Description des brevets d'invention hat Dingler vorübergehend einzelne französische Patentbeschreibungen übernommen.

Im Untershied zur englischen technischen Journalstik, in welcher die mechanische Technik offenkundig cin Ubergewicht gegcnüber naturwissenschaftlichen Themen besaß, liegt das Schwergewicht der nach dem Bulletin de la Société d'encouragement am häufigsten benutzten französischen Literatur auf dem Gebiet der Naturwissenschaften, besonders der Chemic, der Physik und de Pharmazie. Nationale Eigentümlichkeiten sowie die Bedeutung der nach Lavoisier hochstehenden französischen hemisch-nturwisenschaftlichen Forschung machen sich he

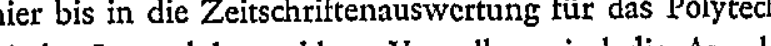
dische Jouna benckbar. Vor allem sind die Annales de chimie et de physique, welche in der zweiten Serie seit I8I6 von J. L. Gay-Lussac und D. F. Arago heraus gegeben wurden, und das seit I8I5 erscheinende Journa de pharmacie von Dingler für seine Zeitschrift ausgewertet worden ${ }^{101}$. Beide Zeitschriften sind wie das erwähnte Bulletin von Anfarig bis zum Ende des Untersuchungseitraumes von Dingler für seine Zwecke benutz worden Den Annales de chinie et de physigue verdankt das Polytechnische Journal vor allem auch zahlreiche Aufsätze des Herausgebers Gay-Lussac.

Alle anderen französischen Periodika haben nur für begrenzte Jahrgänge oder überhaupt nur sporadisch Beiträge zum Polytechnischen Journal beigesteuert. Sehr häufig wurden ab Band 90 bis 1855 mit Ubernahmen von 6 bis $x_{5}$ Beiträgen pro untersuchtem Band ausgewertet die Comptes rendus der französischen Akademie der Wissenschaften, wobei allerdings aufgrund der Dingler' schen Angaben nicht recht ersichtlich ist, um welche Sektion es sich handelt, ferner mit einem oder ein paar, in

${ }^{101}$ Dic Annales de chimic et de physique und das Journal de pharmacie schcinen mit folgenden Beitragsubernahmen in Polyt. Journal auf: Band $x: x(2)$ Beiträge; Bd $x 0: x(x) ; B d$ Is: $3(0) ; \operatorname{Bd} 30: 4(7) ; \operatorname{Bd} 30: 3(3) ; B d 40: 4(3) ; \operatorname{Bd} 50: 4(\mathrm{x}) ; \mathrm{Bd}$

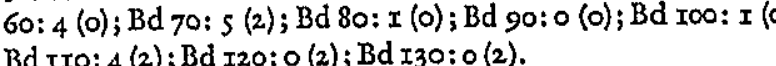


Band 20 sogar mit II Beiträgen der seit $x 835$ erscheinende Moniteur industriel und vereinzelt in einigen Bänden das seit $x 825$ in Paris herausgegebene Journal de chimie medicale.

Immer wieder finden sich in den Bänden des Polytechnischen Journals auch thernahmen aus dem Bulletin de la Société indus zung der Industrie dieser elsässischen Stadt und den Interessen Dinglers entsprechend, sind ihm besonders Artikel entnommen worden zur Textildruckerei, der Textilfärberei, $z \mathrm{u}$ Textilmaschinen und Dampfmaschinen. Weitere französische durch Dingler ausgewertete Periodika waren die $18 \times 9-1826$ erscheinenden Annales de P'industrie nationale et étrangère, ou Mercure technologigue, der $I 826$ in Paris erschienene I'Industriel, der Recuil industriel (Paris a $827-37$ ), dos Joural des conthis unclles usuelles et pratiques (Paris $1825-43$ ) mit allein I5 Beituägen im 50. und 4 Beitagen $\mathrm{im} 60$. Band, dann die Zeitschrift Le Temps (Paris 1829-42), die Annales de la Société polytechnique-pratique und das Echo du monde savant (Paris I834-46) sowie knapp vor Berichtsschluß Cosmos, Revue encyclopédique hebdomadaire $(1852 \mathrm{ff})$, womit die wichtigsten genannt wären.

Eine besondere Nachrichtenquelle für das Polytechnische Journal, die bei der Berichterstattung aus französischen Werken deutlich hervortritt, aber auch bei englichen Referaten besonders in den Miszellen eine Rolle spielt, waren die Sammelwerke sowie die cnzyklopädischen und lexikalischen Produktionen. Karmarsch sieht in ihrer breiten Ausbildung geradezu eine Eigentïmlichkeit der englischen und französischen technischen Litern. tur ${ }^{102}$. So sind in Band Is des Polytechnischen Journa aus dem Dictionnaire technologique das von Joz bis 835 in Paris in 22 Bänden eschion, finf Aufora aber nommen worden nome L'Agriculteur manufacturier (Paris I830-3x) hat Dingle in Band 40 neun Beiträge entnonimen. Dem mehr schullelarbuchartigen Précis de chimie industrielle von Anselme Payen (Paris 1849 ) hat Dingler in Band 120 des Journals drei Beiträge entnommen. Mit zu den wichtigsten Sammelwerken dicser Gattung gehörte Is ánic industriel, revue des inventions francaie ot le gen von Charles Armengaud (Paris 8 (5) Armengaud, Publications ids outils outils et appareils (Paxis $184 x-90)$. Aus dem ersten Werk hat Dingler in Band $x_{20}$ einen Beitrag, in Band $x_{30}$ neun Wnd in Band 138 veezelhn Beiträge, aus dem letzteren Work hat er 3 Beiträge im Band 120 referier

${ }^{102}$ Karmarsch: Geschichte, S. 898.
Ob Dingler die Auswertung aller dieser lehrbuchartigen und lexikalischen Sammelwerke als echten Gewinn an sah oder ob er unter dem Zwang zur Vollständigkeit stand, muß insofern offen bleiben, als er sich gegen Benutzung irgendwelcher Manuels wandte, welcho Che Volz im Polytechnischen Journal vermißt hatten. Emil Dingler schrieb damals r8 34 an Cotta: "Bei aller Vortrefflichkeit und Nützlichkeit können wir natürlich diese Manuels doch nicht dem Journal einverleiben, weil der Zweck desseiben nicht derjenige seyn kañ, bereits bekañte Sachen der Capacität der Gewerbsleute anzupassen, sondern nur, neue Erfindungen und Verbesserungen der nen oder Versuche, wodurch ihre Theorie aufgeklärt

In Ausführung dieses Grundsatzes hat er auch Cott gegenüber angekündigt, $d a ß$ er von einem englischen Hauptwerk dieser Gattung, von Andrew Ure's Philosophy of manufactures "unverzüglich cinige Kapitel, worin zum Theil neue Maschinen und Apparate gut abrebilde und beschrieben sind, für das polytechnische Journ bearbeiten werde «103.

\section{I.3 Deutsche Zeitschriften}

Schr lange in einem bejammernswert geringen Ausma $\Omega$ bzw. zum Teil überhaupt keine Beiträge des Polytechnischen Journals sind deutschen Zeitschriften entlehnt worden. Erst ab dem Ende der 18300 r Jahre, ab etwa Band 7o, wurden zunehmend auch deutsche Zeitschriften von Dingler für den Beitragsteil des Polytechnischen Journals ausgewertet.

Diese Situation entsprach einerseits der Lage der technischen Berichterstattung und der Industrie in Deutschand, war aber andererseits auch Ausdruck besimer. Schrifteitungsprinzipien So schrib J. G. Dister $S$. III der Vorbemerkungen zum daß di Hor Journals, Zeit Zeitschriften, in der Uberzeugung, daß sie unter unsere Landsleuten jene Aufmerksamkeit gefunden haben, welche sie verdienen, unberührt gelassen, und nur zuweilen, wo es das Interesse des Gegenstandes oder des Verfassers orderte, immer aber mit gewissenhafter Angabe de Quelle, benützt" haben. Dies ging so weit ha die Redaktion begründen zu minsen den oder jen

${ }^{103} \mathrm{CA} \mathrm{II} / 5$ 25.xx.1834; $\mathrm{CA} \mathrm{II} / 7$ r5.8.I835

1.04 Polyt. Journal 40 (I83x), S. 26x Anm. 98 ; Bd 45 (I832),
Der geringe Anteil der deutschen Referate im Polytechnischen Journal rïhtt auch daher, daß im Gegensatz ur englischen Publizistik praktisch die gesamte Patentiteratur ausfel. Das proußsche Patentwesen des Vor Worten von Karmarsch "mehr auf Dämpfung als auf Ermunterung der Patentlust ${ }^{105}$. Als einzige deutsche Patente stellte aber Dingler im Journal wiederholt diese preußischen Patente zusammen. Sie fielen jedoch nicht sonderlich ins $\mathrm{Ge}-$ wicht, da in den Jahren I822-I837 nur 272 Patente, also im Durchschnitt ${ }_{77}$ Patente pro Jahr erteilt wurden ${ }^{105}$. Dic Patente Osterreichs nahm Dingler nicht ins Journa auf, "weil sie in beiden Wiener Zeitungen lang und breit auf, "weil sie in bciden

Die Patentschriften der übrigen deutschen Staaten schienen ihm deswegen kein »schätzbares Material« fü das Polytechnische Journal zu bicten, da die in dem jeweiligen deutschen Staat genehmigten Patente alljährlich in den zuständigen Zeitschriften der Zentral-Gewerbe vercine veröffentlicht wurden und mit Ausnahme PreuBens drei Viertel der erteilten Patente in Deutschland inter nicht zur Aus in de Behandlung der Patente und seine Zurückhaltung in der Auswertung der deutschen Zeitschriftenliteratur richtig und sinnvoll waren. Unabhängig von der Qualität de deutschen Zeitschriften und Patente verwies das Polytechnische Journal damit den Gewerbsmann auf diesem Felde auf eine Vielzahl verstreuter Publikationsorgane, während hinsichtlich der ausländischen Literatur es sich min mo ein. Doch muß man hier wohl das Anliegen der Redaktion in Rechnung stellen, hauptsächlich Anregungen aus
dem Ausland aufzugreifen und den deutschen Interdem Ausland aufugreifen

In Befolgung der soeben dargelegten Grundsätze sin unter den deutschen Zeitschriften offensichtlich diejenren am häufigsten ausgewertet worden, die ihrerseits en anteriesen und encen ho Anten an Origen oder die wegen ihres wissench gewerblichen Leserkreis nicht erreichten. So utbernalim Dingler aus den Annalen der Physik und Chemie, welch seit I824 von Johann Christian Poggendorff in Leipzi herausgegeben wurden, mit die meisten Beiträge, bemerkte aber einmal bei einem Aufsatz aus Poggendorff Annalen der Physik und Chemic, daß dieser maus einer huptsüchlich nur für das wissenschaftliche Publicum

${ }^{100}$ Karmarsch: Geschichte, S. $\times 47$

${ }^{100} \mathrm{CAI} / 48$ 6.I2.I822. bestimmten deutschen Zeitschrift" stamme ${ }^{108}$. Eine der weiteren beständiger ausgewerteten Zeitschriften waren die Verhandlungen des Vereins zur Beförderung des Gewerbefleißes in Preußen (seit 1822). Schon im Namen das englische und französische Vorbild erkennen lassend, boten bere vereins doch mehr als nur idas beste Papier und die schonsten Kupferstiche", das ihnen dic zeitgenössische Kritik doppeldeutig nachrühmte. Im Bereiche der Gewerbevereinsliteratur waren diese Berliner Verhandlungen »fast die einzige periodische Schrift, welche für die höhere Industrie, namentlich das Maschinenwesen, Bedeutendes durch Original-Mittheilungen leistet «100. Von Dinglers großem und einzigem echten Konkurenzblatt, geren das er pdurch gründliche Bearbeitung, schnelle

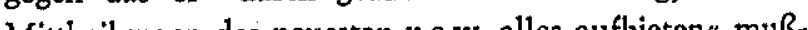
te, "um stets den Vorrang zu behaupten", dem seit $x 835$ in Leipzig erscheinenden Polytechnischen Centralblatt von J. A. Hülsse, hat er ebenfalls wiederholt Beiträge übernommen, und zwar häufig Referate aus regional begrenzten deutschen Organen, doch hat er in Band 83 auch Artikel aus Försters Allgemeiner Bauzeitung durch dic Ubernahme aus dem Polytechnischen Centralblatt seinem Journal einverleibt $t^{170}$.

Aus den Annalen der Chemie und Physik, welche seit I 840 unter diesem Titel erschienen, damals bereits eine längere Vergangenheit hinter sich hatten, und von Justus von Liebig gemeinsam mit Friedrich Wöhler herausgegeben wurden, hat er wiederholt gerade auch Beiträge von Justus von Liebig übernommen ${ }^{171}$. Neben dem gelegentlich ausgewerteten Archiv für Mineralogie, Geognosie, Bergbau und Hüttenkunde, das seit r829 Karl Karstens Be Belin redierte, war das von Richard Felix Marchand und Otto Linné Erdmann seit $x 834$ in Leipzig herausgegebenc Journal für praktische Chemie eine weitere wissenschaftliche chemische Zeitschrift, auf welche Ding-

${ }^{200}$ Polyt. Journal 56 (1835), S. 206 Anm. 27; Poggendorffs Bd $90: x ; B d x 00: 2 ; B d \quad x r o: 2 ; B d \quad I 20: 4 ; B d I_{3} 8: I$.

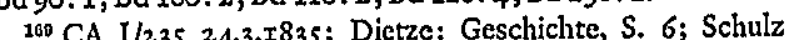
achpresse, S. 35; Karl Karmarsch: Kritische Ubersicht der deutschen technologischen Journalistik, Polyt. Journal 83 $(\mathrm{I} 842)$, S. $x 48 \mathrm{f}$. Aus den Verhandlungen des Vereins zur Beförderung des Gewerbefleißes in Preußen stammten z.B. in Bd xO: $x$ Beitrag; Bd 60: I; Bd 70: 3 ; Bd 80: I; Bd 90: 2 ; $B d x x 0: x ; B d x 20: 3$. ${ }^{170} \mathrm{CA} Y / 257$ 23.x2.x846. Aus dem Polyt. Centrallblatt wurden übernommen z.B. Bd 70: 3 Beiträge; Bd 80: $x_{;}$Bd $90: 2$ $\mathrm{Bd} \times 30: 4 ; \mathrm{Bd} \times 38: 2$.

${ }_{171}$ Ausgewertet 2.B. in Band 90: $3 \mathrm{mal}$; in Bd roo: $2 \mathrm{mal}$, Bd r30: Imal 
ler in seiner Zeitschriftenauswertung, z.B. in Band 80 , I2O, $3 \mathrm{xO}$, zurückgegriffen hat. Von den montanistischen Journalen hat Dingler in den späteren Jahren auch wiederholt Veröffentlichungen übernommen aus der seit 1842 in Freiberg verlegten Berg- und hüttenmännischen Zcitung und dem Montanistischen Jahrbuch des Direktors Tunner zu Leoben.

Häufiger ist von Dingler auch ausgewertet worden das seit $x 834$ erscheinende Wochenblatt für Land- und Hauswirtschaft des Hohenheimer Professors Friedrich Joscph Riecke ${ }^{172}$.

Dingler hat die deutsche Gewerbevereins- und polytechnische Literatur ausfïhrlich beobachtet. Diese lebte aber sehr ausgeprägt vom gegenseitigen Nachdrucken der Aufsätze und wies fast stets nur wenige auswertbare Originalaufsätze auf. Die Diskrepanz zwischen der groBen Zahl der von Dingler trotz seiner Schriftleitungsgrundsätze herangezogenen deutschen Zeitschriften und der geringen Zahl der ihnen entnommenen Referate sowie die kritische Ubersicht von Karl Karmarsch im Polytechnischen Journal über diese Zeitschriften ${ }^{173}$ legt die Ansicht nahe, $d a ß$ es der deutschen technologischen $\mathrm{H}$. ratur am Ende der I830er und Anfang der 1840er Jahre weniger an Zeitschriften als vielmehr an relevanten Originalbeiträgen mangelte. Neben den preußischen Gewerbevereinsverhandlungen hat Dingler von den Gewerbevereinsblättern wiederholt herangezogen die seit $x 8_{34}$ erscheinenden Mitteilungen des Gewerbevereins für das Königreich Hannover, denen er vor allem Aufsätze von Karmarsch entlehnte. Den Polytechnischen Mitteilungen, welche Karmarsch mit dem Tübinger Tochno fessor W. L. Volz seit $m$ in der Auswertung hingegen keine besondere Bedeutung zu, da sie sich nach E. Dinglers Ansicht hauptsächlich an den Kreis der Direktoren und Professoren an polytechnischen Schulen wandten ${ }^{174}$. Die etste Gewerbezeitschrift Deutschlands, der seit $18 x_{5}$ in München herausgegebene Anzeiger für Kunst- und Gewerbefleiß im Königreich Bayern, die dann als Organ des polytechnischen Vereins in Bayern den Titel Kunst- und Gewerbebhet fithre bereits im I. Band des Journals und später wede hot ausgewertet worden.

${ }^{122}$ Ricckes Wochenblatt ausgewertet in Bd 60: $\mathrm{I} ; \mathrm{Bd} 70: \mathrm{I}$ Bd 80: 2; Bd 90: 3; Band roo: I

${ }^{173}$ Karl Karmarsch: Kritische Ubersicht der deutschen nologischen Journalistik Bd 73 (I839) S. 208-234: S. 3xr, S. 370-395, Bd $76(1840)$, S. 52-7I, S.

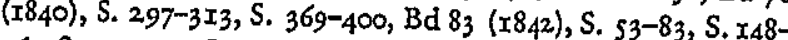
I63, S. 222 I-240, S. 286-330

${ }_{174} \mathrm{CAII} / \mathrm{I8} 20.6 .1844$
Von den übrigen deutschen Gewerbevereinsblättern hat Dingler durch die Ubernahme einzelner Referate besonders auf die der größeren Länder zurückgegriffen. So auf das Innerösterreichische Industrie- und Gewro In Graz erscheinend, auf die Verhandlungen des niederösterreichischen Gewerbevereins (seit I840), die Mitteilungen für Gewerbe und Handel des Vereins zur Ermunterung des Gewerbsgeistes in Böhmen (seit 1834), das Gewerbeblatt für Sachsen (seit 1834), dann die Veröffentlichungen des unter Hektor Rößlers Leitung sehr rührigen großherzoglich hessischen GewerWes nassauischen Gewerbevereins und das Württembergische Gewerbeblatt. Es tauchen aber auch regional oder lokal noch begrenztere Blätter in der Auswertung auf, so das Anfang der 1840 oer Jahre entstandene Berliner Gewerbe-, Industrie- und Handelsblatt, der Jahresbericht des Breslauer Gewerbevereins, der Frankfurter Gewerbsfreund und das Mannheimer Gewerbevereinsblatt. Von den frcien, privat herBlättern hat er unter anderem die Allgemeine Polytechnische Zcitung benutzt, welche seit $x 8_{34}$ als Wochenblatt in Nürnberg von Angehörigen der Familie Leuchs redigiert wurde, dann Rudolph Böttgers seit 1846 erscheinendes Polytechnisches Notizblatt. Dies ist aber nur eine Auswahl von Titeln, die sich noch zu einer längeren Liste vervollständigen ließe.

Beiträge aus nahezu allen diesen Gewerbevereins- und polytechnischen Blättern mit Ausnahme des preußischen und bayerischen Gewerbevereinsorgans haben entsprechend ihren Ersterscheinungsjahren erst ab dem Ausgang der I8zoer Jahre Eingang in das Polytechnische Journal gefunden. Trotz der großen Zahl der benutzten Titel fielen diese Blätter gegenüber den ausländischen Zeitschriften in den Referaten auch deswegen nur sche allmählich ins Gowicht wenige Referte entom mensetze mensetzung der ausgewerteten deutschen Zeitschriften wechselt von Band zu Band des Polytechnischen Journals ganz exheblich, so daß man den Eindruck hat, daß Dingler diese Blätter nicht beständig für jeden Band, sondern nach und nach immer wieder zwischendurch auffearbeitet hat Vielleicht hat or auch gar nich alle dieso Biliter ständig gehalten

Gelegentlich, jedoch nicht sehr häufig hat Dingler Artikel aus Organen technischer Lehtanstalten übernommen, so aus dem Programm der technischen Bildungsanstalt in Dresden und aus den 18 I9 bis 1839 erschienenen Jahrbüchern des polytechnischen Instituts in Wien von Johann Joseph Prechtl. Wiederholt sind von ihm, besonders in den Miszellen, Meldungen und Aufsätze aus Zeitungen, so z. B. aus der Allgemeinen Zeitung abgedruckt worden

Eine gewisse Rolle bei der Herkunft der deutschen Referate im Berichtsteil des Polytechnischen Journals spiclen die Monographien, Schulbuicher und Enzyklopin Im Unterschied zu den französischen technischen Enzyklopädien fällt bei den deutschen Monographien mehr die Zahl der Titel als die Häufigkeit der aus ihnen stammenden Referate ins Auge ${ }^{175}$.

Zusätzlich zu den naturwissenschaftlichen Zeitschriften und den von Dingler benutzten berg- und hüttenmännischen Journalen sowie den Gewerbevereins- und polytechnischen Organen, konnte Dingler dann seit dem Auscang der I84oer Jahre auf die Journale des ncuen Zeitch 作 - und Gewerbezweige spezialisierten Branchenblätter und die berufsständisch ausgerichteten Vereins-Zeitschriften. Es finden sich als Herkunftsangaben von Referaten im Polytechnischen Journal nunmehr $z$. B. die seit I843 in Stutgart erscheinende Eisenbahn-Zeitung, das Archiv für Eisenbahnen, die seit $x 849$ erscheinende Zeitschrift des österreichischen Ingenieur-Vereins in Wien, das Notizenbut de hannor'schen Architekten- und Ingenieurblatt des hannow'sch Archich Vereins, das Rhorographische Journ (seit x854). diesen letzten Untersuchungsjahren finden sich auch häufiger Berichte von Kongressen von Naturforscher und Referate aus Sitzungsberichten der bayerischen, de Wiener und der preußischen Akademie der Wissenscha ten, wobei allerdings auch schon früher öfters Vorträge vor Akademien der Wissenschaften Aufnahme ins Jourmal gefunden hatten. $\mathrm{Zu}$ diesen Referaten und Blättern esellen in Jiesen Jahren auch Verontlichungen

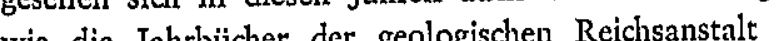
Wic dic Jahrbicher der se Wien oder Benche von Handers und Gewerbekammern. Die wachsende Zahl der von Dingler in diesen letzten Berichtsjahren sporadisch oder permanent herangezogenen Zeitschriften, welche hier keineswegs in voll-

126 Beispicle für Referateübernahmen aus deutschen Monographien bieten Friedrich Julius Otto: Lehrbuch der rationellen

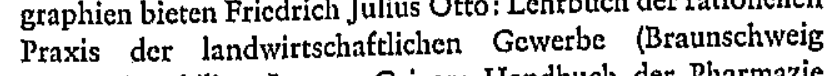
Praxis der landwirtschatchich
I837-55); Philipp Lorenz Geiger: Handbuch der Pharmazie
(Heidelberg I824); Johann Joseph Prechtl: Technologische Enzyklopädie (Stuttgart I830-I855); Karl Karmarsch: HFandbuch der mechanischen Technologie (Hannover I85 5 ); Friedrich Knapp: Lehrbuch der chemischen Technologie (Braunrich Knapp:

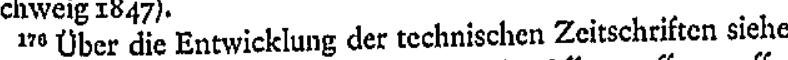
auch Kirchner: Zeitschriftenwesen, 2, S. 48 ff., 205 ff., 224 ff.; Troitzsch: Entwicklung, S. 336i; Schulz: Fachpresse, S. 43 ff. tändiger Aufzählung wiedergegeben sind, und die fachlicherc Ausrichtung dieser Blätter zeigen jedenfalls, daß entsprechend der Differenzierung von Wirtschaft und Wissenschaften vor und um die Mitte des I9. Jahrhunderts die hergebrachte polytechnisch-gewcrbliche Berichterstattung in Auflösung begriffen war.

\section{I.4 Sonstige Länder}

In seinem Gründungsschreiben an Cotta hatte Dingler im Jahre I8I9 die italienische Literatur in einer Reihe mit den englischen und französischen Werken genannt, welche er für das Polytechnische Journal auszuwerten gedachte. Er hat z. B. in Band I, 5, I5, 20 des Polytechnischen Journals wiederholt zwei italienische Zeitschrifand der dann von rofessor Fumagalli erschien, und das Giornale di fisica, 作 1827 in Parma erscheinend. Die Ubersichten über den Stand und die Fortschritte der Okonomie, der Künste und der Gewerbe in Italien und einzelne Gewerbeverfahren scheinen Dingler aber dann doch überzeugt zu haben, daß die italienische Technik der beginnenden Industrialisierung keine entchen vermochte. Die Ausder italienischen Zeitschriften für die Beitrage hör dann praktisch beeeits in den $1820 e r$ Jahren au doch sind besonders in den Miszellen auch später noch Nachrichten aus italienischen Zeitschriften übernommen worden.

Belgien, das gerade für Deutschland in der Frühindustrialisierung eine wichtige Mittlerfunktion in der Weitergabe englischer und französischer Verfahren innehatte, ist bei der Zeitschriftenauswertung, z. B. in Band 5 hach

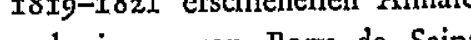
senérales des sciences physigues von Bong de SaintVincent vertreten ${ }^{177}$. Vereinzelt, so in Band $15,49,52$, hat auch eine Übersetzung eines holländischen

den Weg ins Polytechnische Journal gefunden. Weniger für den Beitragsteil als vielmehr gelegentlch für dic Miszellen hat Dingle Gewerbeblatt von Professor Pompeins Bolle and vereinzelt, z. B. in Band ins, aur zurückgerriffen.

Nordarikanische Journale hat Dingler nach eigene Aussage sicher nicht vor I829 bezogen ${ }^{178}$. Uber englische

7 Dinglers günstiges Urteil über diese Zeitschrift, Polyt. Journal 5 (I82I), S. I29, Anm. 66

${ }_{178} \mathrm{CAI} / 179$ I9.12.1829. 
Referateblätter sind aber schon ab dem 2. Band amerikanische technische Artikel im Polytechnischen Journal berücksichtigt worden. Vor allem sind es Aufsätze gewesen aus dem Journal of the Franklin Institute, das seit 826 in Philadelphia herausgegeben wurde, und aus dem von Benjamin Silliman seit I8x8 redigierten American Journal of Science. Aus diesen beiden amerikanischen Journ Journalen hat Dingle kel dirckt ler diese beiden Zeitschriften offensichtlich nicht gehalten $\mathrm{zu}$ haben. Neben einigen anderen amerikanischen Zeitschriften hat er amerikanische Aufsätze wieder indirek über englische Referateorgane erfaßt.

Eine gewisse Hoffnung setzte $x 838$ dic Redaktion auf die Auswertung russischer Blätter. Ein Dr. Widemann sollte Artikel aus dem russischen Journal für ManufakArtikeln aus russischsprachiren Zeitschriften direkt übernommen worden. Daneben gab es noch Übernahmen aus dem französischsprachigen Bulletin scientifique de St. Petersbourg. Uber französische und englische Referatezeitschriften hatte man aber auch hier, ähnlich wie bei den Vereinigten Staaten, hin und wieder wichtige russische Aufsätze indirekt im Polytechnischen Journal erfaßt. Auf diesem Were sind vereinzclt $z$ B auch Band 35, selbst ursprünglich polnische Artikel ins Polytechnische Journal gelangt.

Abschließend muß noch betont werden, daß für dic Nachrichten in den Miszellen zusätzlich noch weitere Zeitschriften und vor allem auch sehr viel häufiger Zeitungen ausgewertet worden sind. Verständlich ist, daß sich in den Miszellen meist noch eine ganz andere Gewichtung der meistausgewerteten Zeitschriften ergibt. Journale, die in den Beiträgen nur wenis Berüksidtigung fanden, sind weren der andersalen vammensetzung in den Miszellen nicht selten sehr viel ausgiebiger ausgewertet worden als in dem stärker nach der techr
teil.

\subsection{Die Mitarbeiter}

Johnnn Gottfried Dingler hat das Polytechnische Journal egründet nicht nur im Hinblick auf die Auswertung der in- und ausländischen Literatur, sondern auch im Vertrauen auf seine und seiner Freunde "eigenthümliche Arbeiten « ${ }^{180}$. Die Originalbeiträge bilden dementspre-

${ }_{180}^{170} \mathrm{CAII} / \mathrm{x}_{3}$ 3x.7.7838, Dietze: Geschichte, S. 6 ${ }^{180}$ Siehe oben Kapitel 2.2. und CA. I/5 8.7.58I9. chend cinen wichtigen Bestandteil des Polytechnischen Journals. Sie errcichen allerdings nur in den ersten $\mathrm{Er}$ scheinungsjahren des Journals und nach der Mitte der I840er Jahre bis zum Ende des Berichtszeitraumes sowie gelegentlich bei einem Band zwischendurch einen Anteil von etwa ro\% oder darüber am Gesamtbestand de Aufsätze.

Während der längsten Zeit des Untersuchungszeitraumes enthielt das Journal nur 3 bis 8 oder 9 Originalbeiträge bei einer Gesamtzahl von oft roo bis I20 Beiträgen pro Band. Manche Bände weisen auch nur einen oder zwei Originalbeiträge auf, und zwei Bände enthalten überhaupt keinen Originalaufsatz ${ }^{18}$. Man kann daher nicht sagen, daß der Anteil der Originalbeiräge am Polytechnischen Jourm im men lite tal sich erst gegen Ende des Berichtszeitraumes die Relationen der Originalartikel prozentual wieder den hohen Anteilen der Zeit des Erscheinungsbeginns nähern, nachdem ihr Anteil durch mehr als zwei Jahrzehnte sogar erheblich abgesunken war. Der Refcratecharakter des Journals blicb während des gesamten Untersuchungszeitraumes crhalten.

Wer veröffentlichte nun im Polytechnischen Journal Originalbeiträge? In den ersten Jahrgängen waren es vor allem Angehörige von Dinglers persönlichem, überwiegend in Süddeutschland ansässigem Bekannten- und Freundeskreis, welche dem Herausgeber Originalartikel beisteucrten ${ }^{182}$. So hat ihm Dr. Wilhelm Heinrich von Kurrer (I78I-I862), mit dem Dingler gemeinsam bereits früher Veröffentlichungen über Textildruckereifragen herausgegeben hatte und nach Dinglers Worten meiner unserer ersten Männer Europens im Fache der Druck-, Färbe- und Bleichkunde «, besonders textildrucktechnische Artikel geliefert ${ }^{183}$. Der Regierungsrat und Kommissär der Stadt Augsburg, Dr. Wirschinger, verbreitete sich über mehr volkswirtschaftliche Themen, und der

${ }^{182}$ Der Begriff Originalbeitrag wurde großzügig verwandt. Den Originalbeiträgen wurden auch alle diejenigen Artikel zugerechnet, welche ohne Verfasser- oder Herkunftsangabe erschienen sind, obwohl bei einer ganzen Reihe von ihnen der

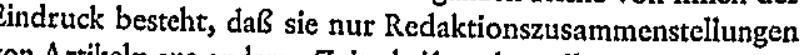
${ }_{182}$ Polyt. Jound anen Zeitschiriften darstellen.

Mitarbeitern, Journal ${ }_{3} 38$ (I855), S. 400. Bei allen denjenigen den diese und weitere beruflichs Conrad Matschoss: Männer der Techniken gewonnen aus: vor allem aus Johann Christian Poggendorff: Bin 1925 ) und iterarisches Handwörterbuch zur Geschichto doraphisch-

Wissenschaften I-5, 2(Leipzig $7863-\mathrm{Ig} 26$ )
Kreisbauinspektor Voit in Augsburg erging sich in den rsten Bänden wiederholt in seinen weitausholenden und fast stets langen Aufsätzen über bautechnische, bauhistorische oder architektonisch-ästhetische Fragen. Stärkeauf dic mechanische Technik in ihren Beiträgen ausgerichter waren Mitorbeter wic Christoph Bernoulli (r78

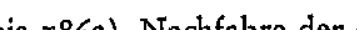
enfamilie und Professor der Naturwissenschaften an de Universität seiner Heimatstadt Basel, wo er hauptsächlich Technologie las, oder der bayerische Oberst:Bergrat, Maschinendirektor und Münchener Honorar-Professo Joseph Ritter von Baader ( $7763-I 835$ ), ein vielseitiger Anreger der Technik und Vorkämpfer des Eisenbahnbaus in Deutschland, für deren Bau er auch im Poly-

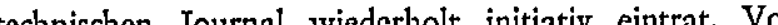
ersten Jahrgängen des Journals wiederholt vertreten Peter Ludwig Marechaux (7764--?), dann der Professor der Mathematil und Physik in München J. L. Späth (1759-I842) und J.A. Buchner ( $1783-\mathrm{I} 852$ ), nachmals Professor der Pharmazie in München. Weitere wichtige Mitarbeiter des Journals in den ersten Jahrgängen waren noch Protessor J. C. Petri in Erfurt, ein Rußlandkenner, der sich besonders iner in teister und Lehrer de Architektur in Augsburg, G. Hävel, der zu Dinglers engerem Bekanntenkreis gehörte.

Es scheint, daß mit Beginn des 3. Jahrgangs die Freunde Dinglers ihren Vorrat an Aufsätzen im Polytechnschen Journal untergebracht hatten. Es sinkt nämlich jetzt die Zahl der Orimalbeiträge ab, und die Paletio der Mitarbeiter wird nach ihrer regionalen Herkunft un der Mitarbeiter wird nach ilhrer region

nach ihrer beruflichen Stellung bunter.
Sozusagen als Echo des Publikums auf das Erscheinc Sozusagen als Echo des Publikums auf das Erscheincn
des Journals tauchen nach den ersten Bänden wiederhol Aufsätze von Lcsern auf, welche meist irgendwelch naturwissenschaftlichen Beobachtungen oder Schilderungen unbekanntor Verfahren einsandten. In Band Iz bringt ein Richter aus der Schweiz einen Beitrag. In Band

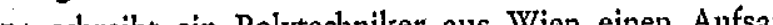
$x_{4}$ schre and der als Maschinen- und Bauingenilun weithin in preußischen Hüttenwesen anerkannte Carl Ludwig AIhaus (I788-r864) von der Sayner-Hütte veröffentlichte einen Aufsatz über Zeichen-Kopiergerätc. Mehrere Au sätze von dem sächsischen Hof-Orgelbaumeister Johan Andr. Uthe in Dresden oder dem Kommandanten de Militär-Akademie in Dresden, Friedrich Christoph Rouvroy, denen weitere Verfasser aus Sachsen und ThürinTrov, dassen gen folgten, lassen exkennen, da das Polyechnise Journal bereits in den ersten Erscheinungsjahren in den mitteldeutschen Raum vorgedrungen war.
Im Jahrfünft $x 825$ bis $x 830$ tauchen neue Namen unter den Verfassern auf. Unter den Professoren haben unter anderen der Bonner Ordinarius für Mineralogie und Geologic Nöggerath (7788-I877) im Journal publiziert genauso wie der Hohenheimer Mathematik- und Physikprofessor Friedrich Joseph Riecke (I794-I876), der Herauser des oben or ähten Wochenblattes für Landund $\mathrm{H}$ a und Hauswirtschaft, und der Marburger Mathematikund Physikprofessor Gerling (7788-x864). Einen Beitrag hat auch geliefert der Professor der Okonomie, Technologie, Chemie usw. Georg Karl Remy aus Keszthely in Ungarn. Sie alle wurden aber keine Dautermitarbciter, sondern waren meist nur Eintagsfliegen im Journal. Vielmehr traten jetzt, ausgeprägter als dies in den crsten Erscheinunssion der Fall gewesen war, wo diese Ent作 wikhng sich allerdings ebenfals bereits abzeichnete, in der Traxis stehende Techniker oder Gewerbsleute als Beiträger in den Vordergrund. Das Polytechnische Journal wurde ein Forum von Bau-Inspektoren, Bauräten, Salinen-Inspektoren, Fabrikleitern, Mechanici, Feuerwerkern sowie Handwerks- und Kunstmeistern. Auch von ihnen scheinen die meisten nur mit einem oder zwei Beiträgen im Journal auf. Einige sind aber Dauermitarbeiter geworden. Dies gilt vor allem für Dr. Ernst Alban (I779I bis I86) aus Pha in Mecklenbure der sich später mit Leidenschaft der Technik, besonders dem Dampfmaschinenbau und dem Bau landwirtschaftlicher Maschinen, widmete. Er hat die erste Maschinenbauanstalt Mecklenburgs gegründet, und von ihm stammen ab Band 27 in der Folge eine große Zahl theoretisch fundierter Originalbeiträge, besonders über Dampfmaschinen und andere technische Probleme. Ein weiterer ukiuntiser beindiger, wenn auch literarisch nicht so literarisch nicht so a Beriranus (x789-x869), Mechanicus aus Bremen und später Ingenieur bei Reichenbach in Blansko in Mahren. Ferdinand Oechsle, Gold- und Silber-Kontrolleur und Mechanicus in Pforzheim, und Dr. Elard Romershausen ( $7784-1857)$ zu Acken an der Elbe, später in Marburg, von Beruf reformierter Pfarrer, stießen ebenfalls damals als Dauermitarbeiter zum Polytechnischen Journal. Eine Reihe von Aufsäzen (a) Würtember m Journal veröffentlicht. In den Jahren vor 1830 hat auch Emil Maximilian Dingler seine ersten Aufsätzc über chemische Themen zum Journal beigetragen. Er wic auch sein Vater haben unter namentlicher Anführung des Verfassers oder als anonyme Redaktion während des gesamten Untersuchungszeitraumes sehr häufig Originalbeiträge im Polytechnischen Journal veröffentlicht. Bei de Redaktionsbeiträgen handelt es sich dabei allerdings 
meist weniger um eigenständige Arbeiten als viclmehr um Kompilationen aus verschiedenen längeren Zeitschriftenaufsätzen zu einem bestimmten Thema.

Uberblickt man das erste Jahrzehnt des Journals, so fällt auf, daß wichtige Mitarbeiter der ersten Stunde wie Marechaux, Wirschinger, Voit, von Kurrer, Petri, vo ren zum Teil überhaupt keine oder nur mehr ganz vereinzelt Aufsätze zum Polytechnischen Journal beigetragen haben. Einzig Joseph von Baader hat dann nach längerem Schweigen zu Anfang der $1830 e r$ Jahre bis z seinem Tod $x 835$ wiederum eine ganze Folge von Originalaufsätzen geliefert, und von Kurrer hat ähnlich wie Bernoulli, mit dem es 1834 eine Verstimmung wegen vermeintlich nicht prompt abgedruckter Beiträge gab, in den späteren Jahren sporadisch mitgearbeitet $t^{184}$.

Im zweiten Jahrzehnt des Journals $183 x-1840$ finden sich unter den wichtigsten Mitarbeitern zum Teil ganz andere Namen als im ersten. Unter den Professorc wird zunächst noch neben Baader in diesen Jahren cin Hauptbeiträger Ludwig Heinrich Zenneck (I779-I859) Professor der Naturgeschichte und Chemic in Tübingen und Hohenheim. Wegen seiner hochwichtigen kritider deutschen technologischen Journ listik, die von Band 76 bis 83 in acht Fortsetzungen im Journal erschien, muß Professor Karl Karmarsch ( 1803 bis 1879), der Direktor der Höheren Gewerbeschule in Hannover, der Vorläuferin der späteren Technischen Hochschule eigens rühmend genannt werden ${ }^{173}$. Anderc Professoren wie der Dorpater Chemie- und Pharmazicprofessor Friedemann Goebel (I794-r85I), Professo Leo in München, Professor Ernst Fabri in Erlangen, Professor der Technologic Cajetan Kaiser (r8o3 Cajetan Kaiser (2803 - ?), der bekannte Münchener Mathematik- und Physikprofessor Karl August Steinhei (r80I-1870) oder der Dresdener Professor Schubert licferten nur einen oder höchstens zwei bis drei Originalaufsätze für das Polytechnische Journal. Einzig der Bascler Chemieprofessor und Erfinder der Schießbaumwolle, Christian Friedrich Schönbcin (x799-I868), veröffentlichte auch in späteren Bänden des Journals hin und wieder Beiträge.

Das Spektrum der übrigen am Polytechnischen Journal durch Originalaufsätze mitarbeitenden Berufsgruppen is wie schon im vorhergegangenen Jahrzehnt wiederum recht praxisnah und bunt. Es finden sich darunter $\mathrm{Me}-$ chanici, Bauinspektoren, Bauingenieure, Baumeister, Brunnenmeister, Münzscheider, Chemiker, Ingenieure,

${ }^{284} \mathrm{CA} I I / 623.2 .2834$
Sekretäre von Gewerbevereinen, Ärzte, Verwalter vo Salinen, Bergwerken oder Fabrikbetrieben sowic Unternehmer. Stärker in den Vordergrund schoben sich jetz in den I83oer Jahren als Mitarbeitergruppe die Lehre an Höheren Gewerbeschulen, Polytechnischen Schulen udgl., welche von den Universitätsprofessoren wegen schwer zu trennen sind. (her an Detring und des. Ausbaus dieser Schulgattung in Deutschland seit den I820er Jahren. Einer der beitragsfreudigsten Mitarbeiter am Journal aus dieser Berufsgruppe wurde in der Folge Dr. Adolph Poppe (I8 13 bis 1894), Lehrer der Technologie und Mathematik in Frankfurt/Main und Sohn des bekannten Technologen an der Univcrsität Tübingen. Vorübergehend hat auch C. Zeller, Lehrer der Landwirtschaft in Karlsruhe und C. Zeller, Lehrer der Landwirtschaft in Karlsruhe und
Sekretär des badischen landwirtschaftlichen Vereins, eine Sekretär des badischen landwirtschaftlichen V
Reihe von Beiträgen im Journal veröffentlicht.

Als wichtige Mitarbeitergruppe während des gesamten Untersuchungszcitraumes haben auch fungiort die Angehörigen von technischen Truppen mit traditionell engen Bezichungen zur Ingenieurtechnik, besonders die

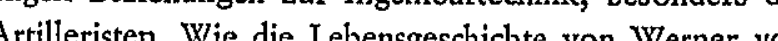
A industrialisierung eine der wenigen Möglichkeiten, einc technische Ausbildung zu erwerben oder technischen Veranlagungen nachgehen zu können, eben der Eintrit in die militärische Laufbahn. Weitere neue Mitarbeite die in diesen $1830 e r$ Jahren mehrere Aufsätze dem Polytechnischen Journal lieferten, waren der Chemiker fü Druck- und Färbekunst in Prog Giunder und Sekretir Pre der rer war ${ }^{185}$, dann der Chemiker J. G. Gentele in Michelbach bei Hall, welcher später einc Fabrik in Stockholm betrieb, und vor allem der Apotheker in Koblenz und nachmalige Extraordinarius für Pharmazie in Bonn, Dr. Karl Friedrich Mohr (I806-I879). Mohr und Gentele blieben auch in späteren Jahren eifrize Mitariter des den bekannteren Erfindern hat Carl Ludwig Freiherr von Reichenbach (I788-I869), Holzverkohlungsfachmann beim Grafen Salm-Reifferscheidt in Blansko in Mähren und Entdecker des Paraffins und Kreosots, in diesen Jahren zwei Beiträge zum Polytechnischen Journal beigesteuert.

Die Originalaufsätze der I840er Jahre lassen in der beruflichen Stellung mancher Verfasser und in den be-

${ }^{185}$ Karl Karmarsch: Ein Lebensbild. Gezeichnet nach dessen hinterlassenen Erinnerungen aus meinem Leben. Mit Ergänzungen von Egb. Hoyer (Hannover $x 880$ ), S. 63 . handelten Themen bereits deutlicher den Ubergang zum Industriezeitalter crkennen. Neu sind jetzt vor allem die Mitarbeiter am Journal, dic in verantwortlicher Stellung im Eisenbahnwesen tätig waren und die nun konkrete Einzelprobleme des Eisenbahnbaus behandelten. So äuBerten sich zu verschiedenen Eisenbahnfagen im Dolytechnischen Journal der Direktor der Taunus-Eisenbahn in Frankfurt, Hofrat Beil, der Maschinenmeister der Düsseldorf-Elberfelder Eisenbahn J. Lausmann oder F. Busse, Bevollmächtigter der Leipzig-Dresdener Eisenbahncompagnie.

Von der Gruppe der Lehrer wird ab diesen x840er Jahten ein Hauptmitarbeiter C. Walther, Lehrer dann professor der pratrischen Mechanit und Maschine dan dc an der Polytechnischen Schule in Augsburg. Er hat zahlreichc, nicht eigentlich kritische oder cigenständige wissenschaftliche Artikel, sondern vorwiegend schlichte Beschreibungen von Konstruktionen, Maschinen oder technischen Vorrichtungen verfaßt. Es war dies eine Art der Darstellung, welche auch bei den übrigen Mitarbeitern des Journals außerordentlich verbreitet war. Diese wird schon dadurch verständlich, daß sich unter den Mitarbeitern nach wie vor zahlreiche Praktiker oder technikbegeisterte technische Dilettanten befanden.

Mit der stärkeren Industrialisierung treten als Mitarbeiter die technischen Beamten, wie zum Beispiel die Salinen-Inspektoren, stärker in den Hintergrund.

Beitragsfreudigste Lieferanten von Oxiginalartikeln in den Jahren $x 84 \mathrm{I}-\mathrm{I} 850$ sind neben Emil Dingler und den soeben erwähnten Busse und C. Walther die alten Mitarbeiter Dr. Mohr, Adolph Poppe in Frankfurt der alte Pforer Romershausen, des rungen an Gewehren und dem geruchlosen Abtritt bis zu Fragen des Elektromagnetismus reichten, sowie Dr. Ernst Alban und J. G. Gentele in Stockholm. Von neuen Mitarbeitern lieferten im ersten Jahrfünft der $1840 \mathrm{or}$ Jahre eine Reihe von Beiträgen L. Seelinger, Fabrikdirektor in Saarbrücken, und G. W. Bichon, ein Freund Emil Dinglers, der bei Justus von Liebig in Gießen Chemic studietce, und L Vi Malinowsky. Mit der VerChe stictir 作 fünft dor I840er Jahre werden auch die Mitarbeiter häufiger, die mehrerc Aufsätze im Polytechnischen Journal unterbrachten. Es lohnt sich nicht, sie alle hier aufzuzählen, wcil sic zu unbekannt und zu viele sind. Jedenfalls befanden sich unter anderem darunter ein $\mathrm{C}$. A. Grüel, Mechaniker und Techniker in Berlin, C. F. Schneitler in Berlin, E. O. Schmidt und August Beringer in Esslingen/Neckar.

Nachdem die Professoren Zenneck und C. F. Schönbein nur melnr vereinzelt am Anfang der I840or Jahre dem Journal Aufsätzc hatten zukommen lassen, stießen im Jahrfünft I846-I850 unter den Professoren neu mit mehreren Beiträgen zum Polytechnischen Journal Friedrich Ludwig Knapp (I8I4-I904), Professor der Technologic und Chemie in Gießen, dann in München und Braunscher (1) wie auch ein Pon den bekannteren Erfindern hat Werner Siemens (x8I6-I892) im Band 97 eine Erstlingsarbeit "Öber die Anwendung der erhitzten Luft als Triebkraft" im Journal veröffentlicht, und de neben seinem Bruder Robert Schlagintweit nachmals bekannte Forschungsreisende und Geograph Herman Schlagintweit (x826-x882) aus München hat sich in Solytechnischen Journal über Meßinstrumente und Lin sen verbreitet.

Im Zeitraum $185 x-I 855$ finden sich unter den Hauptbeiträgen wieder die altbewährten Namen wie Ernst A ban, dessen offensichtlich nachgelassene "Mitteilungen aus meinem Leben und Wirken als Maschinenbauer« in Fortsetzungen im Polytechnischen Journal erschienen dann Dr. Mohr, Romershausen, J. G. Gentele, C. Walther aus Augsburg, L. G. Treviranus in Brünn und mit nachlassendem Eifer Adolph Poppe. Dazu kamen nun als Mchrfachbeiträger Professor G. Decker in Augsburg, der auch schon vorher gelegentlich im Journal veröffentlicht hatte und dessen Beiträge stets mit vielen mathematschen Berechnungen angereichert waren, sowic der Professor an der Polytechnischen Schule in Nürnberg, dann an der Universität in Würzburg Rudolph Wagner (I82 bis I880) und der Chemieprofessor an der Universität München August Vogel jun. (I8I7-I889). Ansonst haben wieder Angehörige der verschiedensten Berufe mit zum Teil neuen, bisher unbekannten Namen Aufsätze zum Polytechnischen Journal beigesteuert. An der regionalen Herkunft der Beiträger ist bemerkenswert, daß jetzt wie auch bereits in den früheren Jahrgängen neben den Südund den Mitteldeutschen aus Hessen und Sachsen auch sehr häufig Nord- und Westdentsche Aufsätze im Polytechnischen Journal veröffentlicht haben. Osterreicher und Deutschböhmen haben seit jeher am Journal als Beiträger mitgearbeitet. Praktisch sind im Journal Mitarbeiter aus dem gesamten damaligen deutschsprechenden oder von Deutschen durchsetzten Gebiet vertreten von der Schweiz bis ins Baltikum und von Straßburg bis nach Lemberg im österreichischen Galizien.

Auffallend, aber erklärlich ist, daß bei den Universitätsprofessoren, welche dem Polytechnischen Journn Aufsutze zukommen ließen, es sich fast ausschließlich um Auturwion zlom sche Chemiker, handelte. Dies resultiert daraus, daß die 
deutschen Universitäten schon in der ersten Hälfte des I9. Jahrhunderts, obwohl es zunächst noch verschieden lich Iehrstible für Technologic gab, zunchmend dic 180. Dic Lend dio hrer und chulen, den Höhleren Gewerbeschulen und den Polytechnika haben erst allmählich diese technische Ausbildungslücke füllen können, und es kennzeichnet eigentlic die Situation, daß es ein Praktiker wie Dingler war, der eine der ersten deutschen technischen Zeitschriften gcgründet hat.

Dic Herausgeber des Journals haben sich nachweislich wiederholt bemüht, neue Mitarbeiter für ihre Zeitschrift zu gewinnen. So schrieb J. G. Dingler I828 an Baron von Cotta, daß ex "viele neue und tüchtige Mitarbeiter gewonnen habe«, und sein Sohn suchte auf einer technischen Reise durch Bölmen und Sachsen Mitarbeiter für das Journal zu interessieren ${ }^{187}$. Beide haben sich auch stets von einer großen Anzahl von Originalartikeln einen cesteigerten Absatz erhofft. Während des Untersuchungs zeitraumes waren es aber schon yon ihrer Zahl her wohl Erfolg der Zeitschrif ntscheidend und nachhaltig bestimmten. So mußte z. B. I862. Emil Dingler enttäuscht bekennen, daß der Absatz des Journals nicht gestiegen sei, obwohl er in diesen Jahrgang eine "ungewöhnlich große Anzahl wichtiger Originalabhandlungen sowohl auf dem mechanisch- als dem chemisch-technischen Gebiete « aufgenommen habe, "welche zum Teil auch viel Aufsehen erregten « ${ }^{188}$.

Die mit Ausnahme einer kleinen Schar von Dauermit arbeitern rasch wechselnden Namen der Beiträger lassen den Schluß zu, daß es zu einer richtigen Pflege eines ausgedehnten und ausgewählten Mitarbeiterkreises und vo Korrespondenten; welche durch ihre Beiträge das $\mathrm{Gc}$ samtgebiet der Polytechnik hätten abdecken können nicht gekommen ist. Die Schwicrigkeit, Dauermitarbeite am Polytechnischen Journal zu interessieren, lag zunächst schon einmal darin, daß sich die Einzeldiszipline zunchmend ihre eirenen fachspezifischen Blüter schufen, dence der Fachopezint in etses en a er damit sicher sein konnte, daß scine Aufsätze zu Kenntnis der Fachkollegen gelangten. Zum Aufbau eines breit gestreuten Mitarbeiterkreises war aber auch di Redaktion des Polytechnischen Journals schon personell nicht in der Lage. Die Gewinnung von Mitarbeitern und ständigen Korrespondenten hätte bei einem Journal mit ener vierzehntagigen Erscheinungsweise und $80 \mathrm{~b}$

${ }^{180}$ Timm: Geschichte, S. 54 ff.

199 $\mathrm{CAI} / 244$ 29.12.1828; CAI/227 27.8.1834.

${ }^{298} \mathrm{CAII} / 57$ 5.2.1862

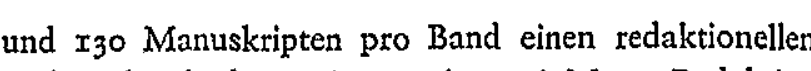
Aufwand erfordert, wie er mit zwei Mann Redaktio und nur mit einem Herausgeber gar nicht zu leisten war. Es scheint, daß schon aus diesem Grund de Estes Organs und die etwas bu zufällige Zusammensetzung des Mitarbeiterkreiscs auch u einer Zeit ungebrochen erhalten blieben, als die technologische Lücke Deutschlands gegenüber England und Frankreich im Schwinden begriffen war und als man schon die Möglichkeit gehabt hätte, seinerseits umfangreicher, als man es getan hat, auf hinreichend leistungsfähige inländische Mitarbeiter zurückzugreifen.

Nach der Betrachtung der Herkunft der Beiträge im Polytechnischen Journal ist noch abschließend zu fragen wie sich das Verbältnis der deutschen zu den ausländischen Artikeln während des Untersuchungszeitraumes entwickelt hat. Das knapp 30 Jahre von 1820 an andauernde deutliche Ubergewicht der englischen Referat ist ausgangs der I840er Jahre durch die damals erreichte, beinahe gleich große Zahl französischer Referate beende worden. Mit Ausmahme der earen Ersheinunging Werter in den r8mer und den frühen 183 ocr Jahren außerordentlich minimal im Polytechnischen Journal vertreten gewesen. Die Anzah der deutschen Beiträge nahm erst seit der zweiten Hälfte der $x 830 e r$ Jahrc spürbar zu. I855, also beim letzten untersuchten. Band, ist das Verhältnis der deutschen, englischen und französischen Beiträge zu jc einem Dritte praktisch ausgeglichen. Die Referateübcrnahmen aus an-

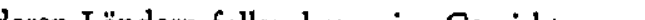
erstmals in anderen Ländern veröffentlicht worden ist.
ent

\section{3 Ưbersetzer}

Die Anmerkungen, fachliche Einwände und Ergänzungen zu den Artikelinhalten von seiten der Ubersetze weisen diese als naturwissenschaftlich und technisch versierte Fachleute aus. Die Ubersetzer fügten den Referaten oft auch ausführliche Bericltigungen oder Hinweise und Schlußfolgerungen für die deutschen Verhältnisse hinzu. Gelegentlich machte der Herausgeber des Journals selbs unter der Bezeichnung des Ubersetzers Anmerkungen besonders i on Verhältnisse betraf ${ }^{180}$.

Trotz der hohen Zahl an Übersetzungen, welche bei der Herausgabe des Polytechnischen Journals anfielen, hatte »dic Redaction des Polyt. Journals ... keine Ưber-

${ }^{190} \mathrm{CAI} / 70$ 3r.T2.I823. sezer angestellt. Einige derselben exhalten ihr Honorar, andere arbeiten olune Honorar mit $\aleph^{100}$.

Nur selten werden die Übersetzer namentlich faßbar. Dem Dingler-Freund und Landshuter Professor Joseph August Schultes verdankt die Redaktion laut Nekrolog zahlreiche Übersetzungen aus den »englischen, französischen und italiänischen technischen Werken und Nachrichten" sowic "vicle der gediegensten Aufsäze und der belehrendsten Notizen «, "ohne daß er seinen Namen jcmals darin geltend machte«. Ebenso hat sein Sohn und Mitredakteur am Polytechnischen Journal bis zu scinem frühen Tod im Jahre I840 besonders Ubersetzungen aus englischen, inärösischen, italienischen und hollëndischen in beiden Schultes wohl die beständigsten Ubersetzer der ersten 20 Jahre des Polytechnischen Journals zu sehen ${ }^{101}$.

In den ersten Bänden des Journals, so z. B. in Band 5, fungierte als Übersetzer von französischen Beiträgen auch der Münchener Professor Marechaux. Ab dem I5. Band und besonders in den Bänden vom Anfang der $1830 e r$ Jahre finden sich dann wiederholt kritische Zusammenfassungen des Uthersetzers im Anschlu an die übersetz-

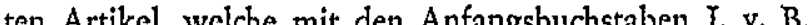
untcrzeichnet sind. Wenn die Vermutung richtig ist, wofür einiges spricht, daß sich dahinter Joseph Ritter von Baader verbirgt, wären von ihm in diesen Jahren zahlreiche englische, aber auch einige französische Artikel übersetzt worden. Aus dem Holländischen hat einmal in Band 49 ein Dr. E. G. Kaiser aus Landshut einen Beitrag übersetzt, und daß russische Zeitschriftenaufsätze ein Dr. Widemann übersetzen sollte, wurde bereits erwälnnt.

Diese wenigen Namen sind die etwas magere Ausbeute auf der Suche nach den Ubersetzern für das Polytechnische Journal. Möglicherweise haben aber auch die Herausgeber in größerem Umfang die Úbersetzungen der englischen und französischen Aufsätze selbst besorgt, und viclleicht hat man sich auch - was nahegelegen hätte - der Lehrkräfte der Polytechnischen Schule in Augsburg für die Úbersetzungen bedient.

Die Schwierigkeit der Ubersetzungen für das Polytechnische Journal lagen dabei vor allem darin, einerseits Ubersetzer mit Sachverstand und Kenntnis der Fachterminologie zu finden. Andererseits sollte in den Ubersetzungen die fachterminologische Nomenklatur nicht zu

100 Polyt. Journal 36 (I830), S. 188

${ }^{101}$ Polyt. Journal 42 (I8 83 I), S. 232 und 78 (r840), S. 80 selhr in den Vordergrund treten, damit diese auch dem einfacheren Gewerbsmann verständlich war ${ }^{102}$. Durch die nuszugsweise und freie Ubersetzung in den unwesent lichen Passagen komprimierte man die Artikel und suchte, sie gemeinverständlicher zu machen. Man stelite damit aber auch hohe Anforderungen an die denkende Mitarbeit und den Sachverstand der Uborsetzer.

\subsection{Mitarbeiterhonorare}

In den Bereich der Aufgaben und Unkosten des Herausgebers fiel nicht nur die Literaturbeschaffung, sondern auch die Honorierung von Mitarbeitern. Es war abe nicht selbstverständlich, daß Dingler jeden im Journal veröffentlichten Beitrag eines Einsenders honorierte. $\mathrm{E}_{\mathrm{r}}$ betonte zwar in den Vorbemerkungen zum xo. und 30. Band des Polytechnischen Journals, daß er alle Originaaufsätze über wichtige Verbesserungen oder neue Brfindungen "auf Verlangen entsprechend« honorieren werde ${ }^{103}$. Er schloß jedoch in der Praxis alle diejenigen Beiträge, deren Veröffentlichung weniger im Interesse des Velmchr des Verfassers lagen, als "nich Honorierung" gecignet von der Bezallung aus ${ }^{104}$.

Dingler hat damit keineswegs alle Originalbeiträge honoriert. Als Begründung für die Erhöhung seines eigenen Herausgeberhonorars führte er im Jalire 1846 unter anderem auch an, daß dic Mitarbeiter, denen es bei den Veröffentlichungen allein um die Ehre gehe, im Schwinden begriffen scien ${ }^{106}$.

Nach seinen sporadischen Angaben in den Briefen an Cotta hat Dingler als Mitarbeiterhonorar etwa 25 Gulden pro Druckbogen veranschlagt. Er war aber zum Bejspiel bei Karmarsch's "Kritischer Ubersicht der deutschen technologischen Journalistik « bereit, mehr zu bezahlen, falls dieser mehr verlangen sollte. In einem anderen Falle meinte er bei einem Text, der keine zwei Kolumnen lang war, daß "das Honorar mit 5 fl honet bezahlt seyn dürfte «100.

${ }^{102} \mathrm{CA}$ II/xoo undatiert.

${ }^{203}$ Polyt. Journal xo (x823), S. I und 30 (I828), S. IV. ${ }_{104} \mathrm{CA}$ I/IS9 $\mathrm{x8.3.1828.}$

100 CA I/246 22.xx.1839; CA II/xoo undaticrt schrieb $\mathrm{B}$ Dingler an Cotta: "Wir bezahlen für den Druckbogen 2-3 Louis d'or Honorar"; $\mathrm{CAI} / 33$ 26.5.I82x. 


\section{Der Inhalt des Journals}

\section{I Der Inhalt der Beiträge}

Seinen Wert erhält das Polytechnische Journal erst durch seinen Inhalt. Der Auswahl und thematischen Zusammensetzung seiner Aufsätze verdankt es wesentlich den Erfolg beim Publikum.

Esfolg beim Publikum.
Es wurde nun versucht, den sich aufgrund der Artikeltitel ergebenden Inhalt der Beiträge des Polytechnischen Journals durch deren Einordnung in die Systematik de Dezimalklassifikation in den Griff zu bekommen. Diese universale Klassifikation hat den Vorteil, daß sic weiteren Kreisen bekannt sein dürfte. Vor allem aber vereinigt dicses System dank seiner hierarchischen Gliede-

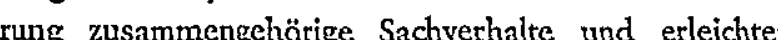
rung zusammengectige Sachverhalte und erleich

damit den Uberblick über den Inhalt des Journals.

Legt man der Inhaltsanalyse die wenig tiefgegliederte, aber für den $Z$ weck einer Ubersichtsorientierung ausreichende Kleinstausgabe der Dezimalklassifikation zugrun$\mathrm{de}^{107}$ und geht man nach der Abfolge der Wissensgebicte dieser Systematik vor, so ergibt sich, daß im Beitragstel des Polytechnischen Journals Fragen der Zoll- und Handelspolitik, des Patentrechts, der Gewerbeordnung und des Handels nur sporadisch behandelt worden sind. In folge der Wiedergbe von Berichten über Aussellugen colge der We ne sowie den Stand und die Fortschritte der Industrie und des Gewerbes in den einzelnen Ländern ist ein mehrmaliges Auftreten von Beiträgen mit diesen Themen zu konstatieren. Der eigentliche Ort der Behandlung allgemeiner wirtschaftspolitischer, handelskundlicher oder wirtschaftsrechtlicher Fragen sowie von Berichten über Gewerbeausstellungen udgl. war im Polytechnische Journal auch der Miszellen- und nicht der Beitragsteil. Aus der Hauptabteilung 5 (Mathematik, Naturwissenschaften) des Systems der Dezimalklassifikation haben die Herausgeber immer wieder zwischendurch einzelne die Herausgeber immer wieder zwischendurch ein

Aufsätze im Polytechnischen Journal veröffentlicht.
So haben die beiden Dingler die mathematische und So haben die beiden Dingler die mathematische und
naturwissenschaftliche Basis der mechanischen und der chemischen Technik durch vereinzelte Aufsätze aus dem Gebiet der allgemeinen Mechanik, der Physik, der Optik, der chemischen Analyse und der Beschäftigung mit chemischen Elementen in ihre Berichtauswahl einbezogen. Von ihnen wurden auch Abhandlungen über astronomische und geoditische Intrumente und die Tempermische und geodhische Insere Temperamineralogische Artikel, jedoch kaum biologische, bota-

${ }^{107}$ Karl Fill: Einführung in das Wesen der Dezimalklassifikation (Berlin, Köln, Frankfurt/M. ${ }^{3}$ x969), S. 5I-To2. nische und zoologische finden sich ebenfalls hin und wieder im Journal.

Ergiebiger wird dic Inhaltsauswertung erst im Bereich der Hauptabteilung 6 (Angewandte Wissenschaften, Medizin, Technik). Die hier unter den allgemeinen Fragen dieser Hauptgruppe cinzuordnenden Patentschauen aus den Zeitschriften sind allerdings nur in den ersten Bänden des Journals im Beitragsteil erschienen. Sie wurden später regelmäßig im Miszellenteil abgedruckt und fielen damit für die Auswertung im Beitragsteil aus.

Naturgemäß hat es Dingler nicht als seine Aufgabe angesehen, medizinische Referate im Polytechnischen Journal abzudrucken. Einzig bei der Lebensmittelüberwachung, der Unfallverhütung, der Brandbekämpfung sowie der Ausstattung von Krankenhäusern und den Heilmitteln hat or in den Randgebieten der Medizin medizinische Aufsätze im weiteren Sinne berücksichtigt. Ohne dic Dampfmaschine als Antriebskraft der zahlreichen neuaufkommenden Arbeitsmaschinen aus dem nunmehr wesentlichsen Werkeff Fisen und ohne Dampteisenbahn ur Dampfeisenbahn und Dampfschiff als neue Transportmittel sind die rasche Produktionssteigerung und Industrialisierung des I2. Jahrhunderts undenkbar ${ }^{108}$. Es war daher Ausdruck der Situation der Zeit, daß der erste große, echt technische Inhaltskomplex des Polytechnischen Journals mit zahlreichen Beiträgen sich mit Dampfmaschinen, Dampfkesseln, Dampflokomotiven, Schiffsdampfnaschinen und sonstigen Problemen der Wärmekneftmaschinen betaßt. Entsprechend den nue sehr allmälich stärker wcr schr alluallch stakker werdenden Ehsatz und Bau der Damphaschinen in Deyschland sind im Polytechnischen Journal die Dampfmaschinen betreffenden Artikel erst ab etwa $\times 825$ spürbar häufiger vertreten.

Recht deutlich läßt sich durch die seit der zweiten Hälfte der $1830 e r$ Jahre einsetzenden Aufsätze und die Häufung der Artikel in den $x 850 e r$ Jahren im Polytechnischen Journal der beginnende Aufstieg der Elektrotechnik verfolsen deren wichtigste wiscenshatiche Grundhen ers im crsten Ditlel des Do. Jahth Gnule Deiner der Telegraph und die zu seiner Anwendung benötigte Energicgewinnung auf elektrochemischem Wege mittels galvanischer Elemente ${ }^{108}$,

Das Gebiet des Maschinenbaus und der mechanischen Technologie wurde im Polytechnischen Journal durch zahlreiche Artikel während des gesamten Untersuchungs-

\footnotetext{
${ }^{\text {108 }} \mathrm{Klemm:} \mathrm{Technik,} \mathrm{S.} \mathrm{273,} \mathrm{36r;} \mathrm{Karmarsch:} \mathrm{Geschichte,}$
S. 209.
}

zeitraumes abgedeckt. Zwischen den Systemstellen 62I.5 (Pneumatische Energie) bis einsclließlich 62x.9 (Spanen(Pnertis de Umformung) der Deimallhssifk tion massicren sich geradezu die Beiträge. Aufsätze, die sich mit Pumpen, Rohren, Rohrleitungen, Hähnen, Ventilen als selbständigc Vorrichtung und als Teile von Maschinen beschättigen, finden sich hier genauso wie solche über Schweißen und Löten, über Kraftübertragung und Hebeeinrichtungen, über die spanende Umformung des Hobelns, Fräsens, Schloifens, S̈̈gens, Drehens und Bohrens sowic Artikel über Hämmer und Pressen.

Mit Ausnahme einiger Beiträge, z. B. über die Grubensicherheitslampen, haben die beiden Dingler den Sektor der gesamten Bexgbautechnik im Inhalt des Journals der gesamten Bergbautechnik im Inhalt des Journals
nicht berücksichtigt. Die Montanistik lag sicher den Interessen der beiden Dingler fern. Wichtiger aber war, daß bereits seit dem $x 8$. Jahrhundert berg- und hüttenmännische Fachzeitschriften herausgegeben wurden, welche im Zusammenhang mit der Errichtung der Bergakademien entstanden waren (Freiberg in Sachsen 1765 , Clausthal 1775 ). Eine Pflege dieses Sektors im Polytechnischen Journal erübrigte sich.

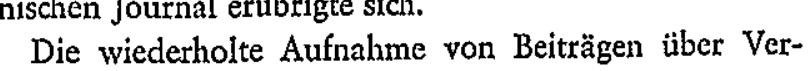
Die wiederholte Aufnalume von Beiträgen über Ver-
besserungen an Flinten oder Pistolen führte zu der Anbesserungen an Flinten oder Pistolen führte zu der An-
sammlung einer gewissen Anzahl von waffentechnischen Beiträgen.

Mit dem Brückenbau beginnt dann ein weiterer inhaltlicher Schwerpunktsbereich, der von der Technik der Verkehrswege, dem Eisenbahnlinienbau, den Eisenbahnfahrzeuren, den Eisenbahnbetriebsmitteln und dem StraBenbau handelt. Aufgrund der Anregungen, die von der ersten öffentlichen Dampfeisenbahn 1825 von Darlingersten öffentlichen Dampfeisenbahn 1825 von Darling-
ton nach Stockton ausgingen, ist es verständlich, daß ton nach Stockton ausgingen, ist es verständlich, daß
sich die Beiträge zu den Schienenbahnen exst ab Anfang

sich die Beiträge zu den Schienenbahn
der $x 8$ 3oer Jahre spürbar vermehren.

Geht man nach der Systematik der Dezimalklassifikation weiter vor, können als folgende Unterschwerpunkte des Inhals des Polytechnischen Journals festgehalten che werden: der Landesked

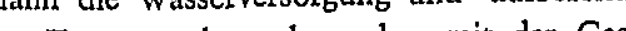
im Zusammenhang besonders mit der Gasbeleuchtung stehenden Probleme der Lichttechnik. Eine Massierung von Beiträgen ergibt sich erst wieder bei den Landfahrzeugen (außer den Schienenfahrzeugen) und den Wasserfahrzeugen bzw. dem Schiffbau; besonderes Augenmerk ist dabei bei den Fahrzeugen den Verbesserungen an den Fahrzengteilen, den Achsen, Rädern und Federn geschenkt worden.

Es entsprach laut Vorwort zum I. Band den Intentionen J. G. Dinglers in dem Polytechnischen Journal auch, die »Land- und Hauswirtschaft" zu behandeln und das Blatt zu einem nützlichen Repertorium für den Landmann zu machen. In der Praxis hat er aber dann doch unzht mur di techniknäheren Bereiche der handwirt berücksichtigt Er beschrankte die Artikel aus diesem Bereich vorwiegend auf landwirtschaftliche Maschinen, Bodenbearbeitungs- und Enntegeräte sowie auf Maschinen und Vorrichtungen zur Aufboreitung oder Zubereitung von Futtermitteln. Es war olne Zweifel eine Folge des I840 erschienenen Werkes von Justus von Licbig: Die organische Chemie in ihrer Anwendung auf Agrikultur und Physiologie, und der damit eingelciteten grundsätzlichen Neubewertung der Dïr critur in Mite Fragen der kanstlichen Dungung und Dungemittel, des Guanos und des Stallmists im Polytechnischen Journal größere Aufmerksamkeit und Berücksichtigung fanden. Für den übrigen Bereich der Land- und Forstwirtschaft des Obst- und Gartenbaus und der Vichzucht kann man feststellen, daß sich zwischendurch immer wieder Beiträge aus diesem Gebiet finden, daß sie aber ticht den Inhalt des Polytechnischen Journals wesentlich bestimm hen Zahl auch nur im ersten Erscheinungsjahrzehnt ins Polytechnische Journal aufgenommen worden. Dingler hat sich in der Folge aus diesem Bereich nahezu völlig zuruickgezogen, der ohnehin durch die landwirtschaftlichen Gesellschafts- und Vereinsorgane schon seit dem letzten Drittel des 18 . Jahrhunderts eine entwickelte Fachpresse besaß109.

Einzig Beiträge über Fascrpflanzen wie Flachs, Hanf, über Seidenroupen- und Schildläusezucht, die als Matetialien fïr die Text auch cine industriew ischafliche Bedeutung besaßen, hat Dingles neben den Düngemitteln im Boreich der Landwirtschaft intensiver berücksichtigt, wie er auch eine Reihe vo Aufsätzen über tierische Produkte aufgenommen hat.

Mit Ausnahme von Artikeln über Kücheneinrichtungen, bei denen es konkret meist um verbesserte Herde oder Ofen ging, hat Dingler nur cin paar hauswirtschaftliche Beiträgc im Polytechnischen Journal abgedruckt.

Völlig ausgespart geblieben sind im Beitragsteil des Polytechnischen Journals Probleme der Betriebsführung, der Betriebswirtschaft und der Organisation von Industrie und Handel. J. G. Dingler hatte zwar I820 im ersten Band des Polytechnischen Journals die Gewerbekunde, die Handels- und Warenkunde als zum Gebiet der Polytechnik gehörig bezeichnet. Das Polytechnische Journal ist in seinem Hauptteil dann aber doch sehr viel

${ }^{100}$ Kirchner: Zeitschriftenwesen 2, S. 33 ff. 
technikbezogener geworden, als es diese Ankündigung hätte vernuten lassen. Bei seiner Inhaltsumschreibung der Polytechnik im Jahre $x 820$ stand J. G. Dingler woh Jahrhunderts, das sich die kaufmännische Schulung de Gewerbetreibenden schr nachinaltig hatte angelegen sein lassen. Die eigentlichen entscheidenden Innovationen sind in der Frühindustrialisierung des I9. Jahrhunder aber dann doch auf dem technischen Sektor erfolgt, und die angehenden Fabrikantenunternehmer dieser Ära sahen sich auch in erster Linie mit technischen Problemen konfrontiert, während sie dic kaufmännische Seite de

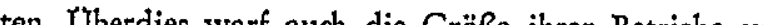
ten. Uberdies warf fangreichere betricbsorganisatorische Fragen noch gar

Aus dem Systembereich "Betriebsführung und Organisation von Industrie, Handel und Verkehr» hat Dingle einzig nur von dem hier reichlich isoliert olne rechten Zusammenhang zum Oberthema stehenden Gebiet »Graphische Gewerbe, Druckerei, Verlag, Buchhandel« ein Reihe von Aufsätzen im Polytechnischen Journal veröffentlicht.

Nach dem Dampfmaschinen- und allgemeinen Ma schinenbau, dem Schiff- und Fahrzeugbau beginnt m der Dezimalklassifikations-Systemstelle 66 (Chemisch Technik, Chemische Industric, Verwandte Industriezwe ge) der nächste große Inhaltsschwerpunkt des Polytechnischen Journals. Noch ehe man in Deutschland von de Existenz einer entwickelteren chemischen Industrio sprechen konnte, welche mit erheblicher Vetzö ïber En Jate des I. Jahrhunderts verhaltismanig unvermittelt ihr rasches und spektakuläres Größenwachstum begann, sind in Polytechnischen Journal bercits zahlreiche Aufsätze zur Seifenherstellung, der Erzeugung anorganischer und organischer chemischer Produkte, über Mineralfarben, dic Technologie der Gase und der Brennstoffe veröffentlich worden. In einer beachtlichen Anzahl von Beiträgen sind im Polytechnischen Journal dann weirerc Aibeiten schienen, welche über vabesente techische

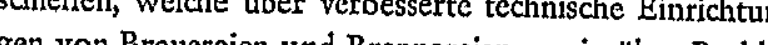
gen von Brancien und Brenner me der Herstellung alkoholischer Getränke handelten. Dem wirtschaftlichen Aufsticg der Rübenzuckerfabrikation in der ersten Hälfte des 19 . Jahrhunderts, besonders seit den 183 oer Jahren, entspricht die häufige literarische Beschaftigung im Polytechnischen Journal mit den Fragen der Rubenzuckerherstellung und der

${ }^{200}$ Karmarsch: Geschichte, S. 829ff., 238 ff. winnung von Stärke, Sirup und Melasse ${ }^{200}$. Probleme de Brotzubereitung, der Getreidclagerung und -verarbe tung der Mïllerei und verbesserten Mühleneinrichtu(a) wiederholt angeschnitten worden. Ebenso wurden Fragen der Konservierung von Nahrungsmitteln, der Kerzenherstellung und der Ol- und Fett- sowie Wachsgewinnung behandelt. Durch ihre Reforateauswahl sind die beiden Dingle auch wiederholt cingegangen auf Fragen der Glasindustrie und Glastechnologie und auf die Herstellung von Feinkeramik, Grobkeramik und Porzellan.

Von den weiteren, mchrmals berücksichtigten Themen ist dann weniger der Bereich der Anstrichmittel und de malerhandwerklichen Anstrichtechnik als vielmehr de Scktor Farbstoffe und Färbcrei in sehr enger Beziehung zu sehen zu seiner Anwendung in der textilindustriellen Färbetei und in seiner Herstellung zu den chemischen Erzeugnissen, wobei jedoch die Zeit der aufsehenerregenden Entdeckungen und die Zorstellung im grosen de Eroßn der knapp nach Berichtsschluß einsetzte. Fragen der Metallurgic, der Eisen- und Metallverhütung, der Roheisen- und Stahlgewinnung und der Hochöfen haben Vater und Sohn Dingler in ihrem Journal bereits in den ersten Jahrgängen in ihre Berichtsauswah einbezogen. Verstärkt sind sie jedoch auf diesen Themenkreis erst in der Zeit vor und um die Mitte des $x 9$ Jalurhunderts cingegangen, als dic bisherige, praktische Rührigkeit und dem Brennmaterial Holzkohle verpflichtete Eisenverhüttung in Deutschland unter dem Vorbild und der Hcrausforderung der englischen Konkurren gezwungen war, zu Kokshochöfen und modernen Verfahrensarten übcrzugehen ${ }^{200}$.

Verhältnismäßig wenig und überdies offensichtlich mit nachlassender Intensität gegen Ende des Berichtszeitraumes in der Berichterstattung gepflegt haben die beiden Dingler den Bereich der verarbeitenden Eisen- und Metallgewerbe und der Holzindustrie und HolzverarbeiMetallgewerbe und der Holzindustrie und Holzverarbei-
tung. Diese betriebsreichen, handwerklich/kleinbetrieblich strukturierten Gewerbezweige blicben allerding auch im 99. Jahrhundert trotz einer Reihe neu erfundener Arbeitsmaschinen eine der stärksten Domänen de praktischen Handwerker und Empiriker, 50 da auch nicht vid an techis the lichen Rrfindungen zu berichten gab. Die technische Berichterstattung hatte damit gerade zahlreichen kleinen Handwerkern und Gewerbetreibenden, zu deren Schulung und Informierung über dic Fortschritte der Technil dicse polytechnischen und Gewerbevereinsblätter in erster Linie gegründet worden waren, mit am wenigsten zu bieten.
Hat Dingler die Papierindustrie, die durch die Erfindung der Papiermaschine I799 und 2844 des Folzschliffs in der ersten Hälfte des I9. Jahrhunderts sich im technischen Umbruch befand, mit einer Folge von Referaten berücksichtigt, so ist anzumerken, daß Probleme der Gerberei und Lederindustric zwar wicderholt im PolyGerberei und Lederindustric zwar wiederholt im Poly-
technischen Journal behandelt worden sind, daß diesc aber auch kein bevorzugtes Berichtsthema darstellten. Der Grund für diese geringe Berücksichtigung der Gerberei und Lederherstellung dürfte darin liegen, daß dic entscheidenden Neuerungen und die Industrialisierung dieser Branche erst in der zweiten Hälfte des I9. Jahrhunderts erfolgte.

Eigenes Interesse des Textilfabrikanten Dingler an der Textilindustrie und die technische und wirtschaftliche Bedeutung dieses ersten umfassend mechanisierten und besonders im entwicklungsführenden England ältesten hochkapitalistiscl/großindustriellen Industriezweiges haben zu einer dem Maschinenbau und der Chemie ähnlichen Schwerpunktbildung textilindustrieller Beiträge im Polytcchnischen Journal geführt. Neben der Tex-

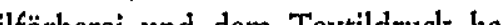
tilfärberci und dem Textildruck hat dabei das weitau stärkste Interesse gefunden dic Technik der Textil-
maschinen mit vereinzelt bis zu über zehn Beiträgen maschinen
pro Band.

Von den weiteren Themen sind im Polytechnischen Journal häufiger behandelt worden Fragen der allmählich das Versuchsstadium überwindenden Kautschukund Guttapercha-Gewinnung und -vorarbeitung und die Technologie der Bearbeitung von Steinen. Von dem feinmechanischen Gewerbesektor sind bei der Berichtsfeinmechanischen Gewerbesektor sind bei der Berichtsauswahl wiederholt berücksichtigt worden die Uhrmahöheren Anforderungen an die Meß- und Regeltechnik wichtig werdende Meßgeräte- und Meßinstrumentenherstellung, dann die Vervielfälttigungsmaschinen und -geräte zum Schreiben, Kopieren und Drucken sowie der Musikinstrumen, Kopier

Sehr häufig wurde im Polytechnischen Journal cingeangen auf Probleme der Herstellung und dic Technik der Verbrennungslampen, der Ollampen und der Gasleuchtkörper, die bis zur Einführung der elektrischen Beleuchtung einen dcr folgenreichsten Fortschritte im Beleuchtungswesen darstellten.

Olne eigentliche Schwerpunktbildung in irgendeinem Sektor sind ferner von. Dingler in seinem Journal gelegentlich oder öfters Themen behandclt worden aus dem Bereich der Schlosserei, der Sattlerei und der Schuhmacherei, der Schirmherstellung, der Versilberung und Vergoldung, der Bürogeräte und der Hütefabrikation.
Beim Bauhandwerk verdichten sich die Aufsätze geringfügig $\mathrm{za}$ den Baustoffen und den Bauteilen. Es ist aber hier genausowenig zu einer echten inhaltlichen Schwerpunktbildung gekommen wie bei den sanitären nos ugs und des Schornsteinbaus. Aus dem Inhaltskomplex der Hauptabtcilung 7 der Dezimalklassifikation (Kunst, Kunstgewerbe, Photographie, Musik, Spiel, Sport) haben die beiden Dingler ebenfalls einige wenige Themenbereiche in ihr Journal aufgenommen. So haben sie eine Reihe von Refcraten abgedruckt, welche über Zeichengeräte und Zeichenmittel sowie über Drucktechniken, besonders auch über die im lezten Jahrzehnt des I8. Jahrlunderts in In stärkerem Umfang haben sie dann seit den ersten x840er Jahren Technik, Ausrüstung und Grundlagen der soeben aufgekommenen Photographie in ihre Berichtsauswahl einbezogen

Diese Inhaltsanalyse ist unter der Einschränkung zu betrachten, daß der der Aufschlïsselung zugrundeliegende Auszug aus der Deximalklassifikation nur eine sehr aro Aur sierten Reihe von Problemen und auch Wahlmöglichkeiten bei der Zuordnung einzelner Beiträge zu bestimmten Systemstellen, auf die hier nicht näher eingegangen werden soll. Für den vorliegenden $Z$ weck, bei dem es weniger um die einzelne Fein-Systemstelle als vielmelnr um die Herausarbeitung der Fachgebietsgruppen ging, vermittelt aber diese Inhaitsaufschlüsselung doch ein ausreichendes und in und in

Das von Dingler angestrebte Ziel der Viclfalt des Inhalts ist ohne Zweifel erreicht worden. Für Lücken oder Aussparungen in der Berichtsauswahl lassen sich durchwcgs plausible Gründe anführen. Die vorrangige Auswertung der englischen und französischen Literatur wirkte sich auf das Polytechnische Journal dadurch positiv aus, daß es ansesichts der industriellen Rückständigkeit Deutschlands in seinen Referaten gegenüber der tatsächlichen Wirtschaftssituation in der Regel einen zeitlichen Vorsprung besaß. Betrachtet man allerdings die in den ersten Jahrgängen des Journals nur zögernd cinsetzende intensivere Berichterstattung auf dem Gebiet der Dampfmaschinen, so hat man den Eindruck, daß Dingler in den ersten Jahrgängen mit seiner Nachrichtenvermittlung zwar mit den industriellen und technischen Fortshriten Deutschlands loonform ging do $B$ er ihnen a dic in aber auch nicht wesentlich voraus war. Dic in Artikellänge und Themenauswahl unausgeglichene Zusammen- 
Karmarsch, daß die ersten Jahrgänge des Polytechnischen Journals auch seine schwächsten waren ${ }^{201}$. In Stil und Inhalt hat sich aber dann das Polytechnische Journal rasch konsolidiert. Bemerkenswert ist, daß der Eintrit des Sohnes Emil Dingler in die Redaktionsatheit im Jahre 830 sowic dic tionsarbeit durch ihn in der Folgezeit keine augenblicklich sichtbare entscheidende Änderung des Inhalts des Polytechnischen Journals zur Folge hatte.

\subsection{Der Inhalt der Miszellen}

Bei der Besprechung des Inhalts der Miszellen kann man zunächst zwci größete Gruppen von Berichten voranstcllen, welche mit einiger Regelmäßigkeit im Miszellenteil aufscheinen, nämlich die Zusammenstellungen von Patentgenehmigungen und die Literaturberichte.

Das Polytechnische Journal hat nicht nur im Beitragsteil ausführliche Beschreibungen einzelner Patentverfahten wiedergegeben. Es hat von Anfang an Übersichten über die neuerteilten Patente in England, Schotthond, Frankreich, den Vereinigten Staten und Preußen veroffentlicht Die Patentshau Sten in den esen

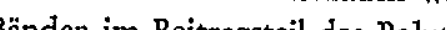
Bänden im Beitragstell des Polytechnischen Journals untergebracht. Ab Band Io wurden sie regelmäßig im Miszellenteil veröffentlicht

Vollständigkeit in der Wiedergabe aller neuen Patente ist im Polytechnischen Journal offensichtlich nur bei den englischen Patenten angestrebt und erreicht worden. Nahezu in jedem Heft des Polytechnischen Journals finden sich die Zusammenstellungen der in den letzen Mon vor Erscheinen des jeweilion atente Ab Band as baben die Herule Patente. Ab Band 25 haben die Herausgeber auch Verzeichnisse der in England nach $x_{4}$ Jahren Rechtsschutz verfallenen Patente mitgeteilt. Nach den Worten Emil Dinglers war das Polytechnische Journal die einzige Zeitschrift in Deutschland, "welche alle englischen patentierten Erfindungen mittheilt, und da man gegenwärtig gar kein Journal - nicht einmal in Frankreich oder England - besitzt, welches dafür ein vollständiges Reperg. wäre, so sehen sich die deutshen Shifteller Technik senthigt ster das polythische Jou technik genothigt, stets das polytechnische Journal zu citieren, um nicht auf ein Heer von fremden Zeitschriften verweisen zu müssen, die man nicht einmal auf den taatsbibliotheken vorfindet ${ }^{202}$

Nicht unbedingt in jedem Heft, aber doch in verhältnismäßig dichter Folge finden sich meist am Schluß der jeweiligen Miszellen Litoraturzusammenstellungen über

\section{${ }^{201}$ Polyt. Journal $2 \mathrm{I}_{4}\left(\mathrm{x}^{2} 8 \mathrm{74}\right)$, S. II}

${ }^{202} \mathrm{CA} \mathrm{II} / 3$ 20.2.I834; Dictze: Geschichte, S. 6 die neueste polytechnische Literatur. Berücksichtigt wurde dabei vor allem die neuerschienene englische, französische, italienische und deutsche polytechnische Literatur. Vereinzelt wurden auch holländische und porturesischo Titel mitaufer ökonomische Titel miteinbezogen. Es handelt sich bei diesen Schrifttumsverzeichnissen um reine Bibliographien; sie beschränken sich nur auf die Anführung der Titel und der bibliographischen Angaben. Wohl aus der Überlegung heraus, daß für den Leserkreis des Poly. technischen Journals zur Lektüre ohnehin nur deutschsprachige Titel in Frage kämen, wurden einzig und allein bei der neucsten deutschen und ins Deutsce ubero watur wichtige Werke auch kritisch rezensiert, oder es wurde zumindest ausführlich über ihren Inhalt referiert. Naturwissenschaftliche Werke waren von dieser Berichterstattung normalerweise ausgeschlossen ${ }^{203}$.

Zusätzlich zu den Patent- und Literaturübersichten waren die Miszellen die bevorzugte Wiedergabestelle für die Nachrichten über die Aktiviẗten der vaschictenen Gewerbevereine: Über Preisausschreiben, Gewerbeausstellungen und Preisverteilungen. In den Miszellen wurden auch überwiegend dic Berichte über den Zustand der Gewerbe und der Industrien in den einzelnen Ländern abgedruckt. Statistische Angaben über Einwohnerzahlen und Bevölkerungsbewegungen, über Wirtschaftsproduktion und Handelsvolumen und die Wirtschaftskraft der einzelnen Iänder Regionen oder Sẗ̈dro finden sich in ihnen genauso wie Ertrassechnungen für einzelne Wirtschaftssparten

In den Miszellen wurden Hinweise gegeben auf neue Zollsätze und die Mautgesetze und Mautpraxis einzelner Länder. Es wurden die Vor- und Nachteile des Freihandelssystems angesprochen und die Einführung neuer Maße und Gewichte kolportiert. Es waren dies alles Nachrichten, die für den praktischen Gewerbsmann von unmittelbarem Wert waren

Mit dem fortschreitenden Verkehrsausbau wurden in den Miszellen auch Nachrichten verbreitet über Eisenbahn-, Kanal-, Tunnelbauten und Straßenausbauten. Sehr häufig wurden in den späteren Bänden auch Nachrichten verbreitet über technische Unglücksfälle und Verkehrsunfälle, über Entgleisungen von Eisenbahnen, Explosionen von Dampfkesseln, Schiffsunglücke udgl., wobei diese Meldungen über den Sensationswert himausgehend den Zweck verfolgten, $d$ Urachen $Z$ Veres technischen Einrichtungen anzuregen. Notizen über Ge- winne ausländischer Eisenbahngesellschaften sollten offensichtlich zur Gründung ähnlicher Gesellschaften in Deutschland anspornen. In den Miszellen hat Dingler auch wiederholt kurze Beriche über neue polytechische Schulen verbreitet. Verhältnismäßig häufig sind in den Miszellen landwirtschaftliche Themen angesprochen: über landwirtschaftliche Anbauflächen, über Anbau, Ertrag und Nutzung einzelner Pflanzen oder über Schädlingsbekämpfung. Die Miszellen teilten auch Nachrichten übor Rohstoffunde oder die Höhe der Arbeitslöhne mit und verglichen die Leistungsfähigkeit und den Bildungsstand der Arbeiter in den einzelnen Iundern SozialFragen wurden allerdings selten angesprochen. Wiederholt hat Dingler in den Miszellen Nekrologe und Ehrenbezeugungen abgedruckt.

Diese soeben wiedergegebene Aufzählung stellt die Eigentümlichkeit der Nachrichtenzusammensetzung im Miszellenteil des Polytechnischen Journals in den Vordergrund. Sie darf aber nicht darüber hinwegtäuschen, daß der Großteil der Nachrichten im Miszellenteil die gleichen Themenkreise wic der Beitragsteil zum Gemenstand batte, nur daßs stand hatte, nul hafter Form wiedergegeben wurden. $\mathrm{Da}$ eine feste inhaltliche Abgrenzung zwischen Miszellenteil und Beitragsteil nicht bestand, läßt sich auch daraus ersehen, daß z.B. in Band $I 6$ ein in Fortsetzung abgedruckter Aufsatz im Beitragsteil begonnen und im Miszellenteil beendet worden ist.

Insgesamt läßt sich als Tendenz feststellen: Die Miszellen stellen cine Ergäzung und Fnthasung des Beitragsters da dic Gewerberebenden infomieten Uhe allec Tren der Handels- und der Warenkunde. Sie ergänzten den Beitragsteil auch dadurch, daß sie die wirtschaftliche Nutzanwendung der neuen Erfindungen ansprachen und den Polytechniker über die neueste Literatur informierten. Sie entlasteten den Beitragsteil, indem sie einfache Sic

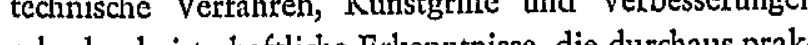
oder landwitschafliche Brke tischen Nutzwert hatten, aber einen längeren Beitrą nicht lohnten, dem Publikum zur allgemeinen Unterrichtung und zur Anwendung zur Kenntnis brachten. Manchen der Miszellennotizen ist nur ein Kuriositätenwert zuzusprechen.

\subsection{Anmerkungen, Zusätze und}

Redaktionsnotizen

Es ist bereits wiederholt darauf hingewiesen worden, daß zum sachlichen Referat im Polytechnischen Journal noch fachliche und kritische Anmerkungen in Fußnoten, Hinweise und Zusätze von seiten der Redaktion oder der Ubersetzer gemacht wurden. Obwohl meist anonym bleibend und im Inhalt an das Referat anschließend oder aus diesem hervorgehend, kommt diesen Meinungsäußerungen nicht selten ein selbständiger Nachrichtenwert zu. Dicse Hinweise, Bedenken und Ergänzungen stellen oft erst den fachlichen Bezug der Artikelinhalte zu den deutschen Gewerbsverhältnissen her oder berichten übe Anwendungserfahrungen. Besonders manche der Zusätze, zum Beispiel die von J. v. Baader anfangs der 18300 Jahre, weiten sich im Anschluß an die Referate durch J ihre Länge

Diese Zusätze und Anmerkungen sind in den Bänden ab Mitte der I830er Jahre seltener geworden, bzw. sic beschränken sich jetzt häufiger auf Hinweise weiterführender Literatur, oder sie verweisen auf ähnliche Artikel im Polytechnischen Journal. Dagegen werden in den späteren Bänden diejenigen Beiträge häufiger, in denen dic Redaktion die wesentlichen Ergebnisse aus Zeitschrifurberichten zusammenfass jetzt auch wiederholt den Referaten einführende ode erklärende Einleitungen voran, die zum Wesentlichen eines Beitrags hinführten 204. Emil Dingler hat auch in den späteren Bänden selbst wiederholt Zusätze zu Referaten gemacht und mit E. D. abgezeichnet.

Jedenfalls beschränkte sich die Redaktion des Polytechnischen Journals keineswegs nur auf die stumm Auswhl der Referate. Für sie war durch diese kommenreferierende Taigkent mit der Herausgabe des Jou

Der politisch aktive Johann Gottfried Dingler sah überdies in seinem Journal »kein Zeitungsblatt, das alle Farben spielen muß « ${ }^{200}$. Er hat dic Anmerkungen und die Miszellen daher auch als Plattform zur Verkündung seiner wirtschaftspolitischen Ansichten benutzt. Seine wirtschaftspolitiche, besonders handelspolitische Auffaswits sung, laßte er in de Mindle cinmal zun werk durch Deutschland und Verboth aller fremden Waaren,

204 Polyt. Journal 64 (I837), S. 6r: "Wir stellen im Folgenden die wesentlichen Ergebnisse dieser interessanten Arbeit zusammen.« Polyt. Journal 66 (I837) S. x68: "Wir geben daher in Folgenden eine gedrängte Beschreibung der Art, wie Castel experimentierte, und fügen dic von ihm gewonnenen Resultate

${ }^{205}$ Polyt. Journal 33 (I829) S. 478 . 
was die Bundesstaaten vor gänzlicher Verarmung retten kann ${ }^{200}$. Er trat für Freihandel im Innern Deutschlands sowie für Prohibitivzölle gegen die Einfuhr ausländischer Waren ein und beriüre ausländische Direttinvestitionen in Deutschland, da damit die Erziehung der Deutschen zur Industrie befördert würde ${ }^{207}$. "Philosophischer Schnikschnak, theologischer Mystizismus, Fanatismus und Jesuitismus und peristische Alfanzerei" waren ihm verantwortlich für den rïckständigen Zustand der Industrie in Bayern und in den meisten deutschen Staaten ${ }^{208}$. Diese Überzeugung, verbunden mit entschiedener Fortschrittsgläubigkeit verlieh seinen Kommentaren nicht sen einen ungeöhnlich ageressiven Ton und fïhto lische Frömmigkeitsformen ${ }^{200}$. Unter dem andersgearte-

ten Emil Dingler hören diese zum Teil recht subjektiven Urteile und politisierenden Kommentare auf. Er beschränkte sich nur mehr auf rein sachliche Anmerkungen, Jedenfalls widerlegen gernde diese Auslassungen $J G$. Polytechnischen Journals die Ansicht von Troitzsch, daß Dingler, "bei aller Treuc zur Sache, aus wirtschaftlichen Gründen, allein um den Absatz seines Blattes zu sichern, mancherlei Zugeständnisse im Hinblick auf den Inhalt machen" mußte. Gerade in der auch von Dingler ausgenutzten Ungebundenheit gegenüber den anstehenden wirtschaftspolitischen Frasen und der von Vereins- und Mitgliederder der freien polytechnischen Bcrichterstattung gegenüber

6 Preis, Herstellungskosten, Auflage und Absatz

6.I Preis und Herstellungskosten

6.I.x Der Preis des Journals

Ohne genaue Kenntnis und unabhängig von der detaillierten verlegerischen Kalkulation über Herstellungskosten, Honoraranteil, den Vertriebskosten und allgemeinen Unkosten schätzte J. G. Dingler die Höhe des Verkaufspreises, der den Abnehmern des polytechnischen Journals zugemutet werden könnte, anders Jerleger Noch ehe das Verlegcr. Noch che das orste Heft erschienen war, meinte er Herausgeber, den Ress des Dolytechnischen Journa würde er auf $x 2 \mathrm{fl}$ pro Jahrgang festsetzen, »der Absat würde dann auch verhältnismäßig größer seyn; vorzüglich bei Oekonomen ¿ $^{211}$. Der Verlag kalkulierte jedoch dic Marktchancen und Unkosten anders und legte den Bezugspreis des Polytechnischen Journals bei $x 6 \mathrm{fl}$ jährich fest. I82I ließ der Herausgeber wiederum vorne men, es hatten sich viele seiner Korrespondenten dahingehend geäußert, daß es zur Vermehrung des Absatzes besser wäre, den Preis des Jahrgangs auf $\mathrm{I}_{2} \mathrm{fl}$ herabzuetzen, "diese Aeußerungen sind Folge der geldarme Zeiten, worinnen ihr Grund zu suchen ist ${ }^{212}$. Obwoh

Polyt. Journal I3 (x824), S. 270 f. Anm. To3.

${ }^{200}$ Polyt. Journal is ( $(2824)$, S. $49 x$.

${ }^{209}$ Polyt. Journal 25 ( $(827), S .87 \mathrm{f}$

${ }^{200}$ Polyt. Journal 12 (I823), S. 425 Anm. I633; Polyt. Journal

$I_{3}(x 824)$, S. 255 und S. 392 f. Anm. I63 und Anm. 205 U. 207.

${ }^{210}$ Troitzsch: Entwicklung, S. 332; Schulz: Fachpresse,

S. 32 ff.

211 CAI/ro 3.Ix.r8I9.

${ }^{212} \mathrm{CA1} / 32$ 9.5.x82.
Dingler in diesem Brief $182 \mathrm{I}$ auch schrieb, daß er überzcugt sei, daß der Preis von I $6 \mathrm{fl}$ nicht zu hoch sei, hat er jedoch anläßlich seines Zeitungsplanes im Jahre I825 wiederum gefordert, daß der Preis der Zeitung höchsten bei II $\mathrm{fl}$ festgelegt werden dürfe, damit das Blatt den Gewerbsleuten, für die es zunächst bcstimmt sei, auch zugänglich sei ${ }^{213}$. Genauso schlug er bei den Bemühungen um die Absatzsteigerung r82I vor den Behörden

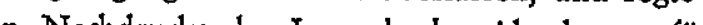
n, Nachdrucke des Journals den Abnehmern für I2 bzugeben ${ }^{214}$

Diese wiederholten Anregungen Dinglers zur Senkung des Abgabepreises scheinen insofern einen realen Hintergrund gehabt zu haben, da sich nach der Berechnung vo Schulz ergibt, daß gegenüber einer vergleichbaren naturwissenschaftlichen Zeitschrift aus dem Jahre 1790 das Polytechnische Journal $\gg d e m$ Nennwert nach teurer im Bezug war. Die Preislage der späteren Blätter, insbesondere des ebenfalls führenden Polytechnischen Centralblattes (seit 1835 ), entsprach überdies demjenigen Bezugspreis, der sich ergeben hätte, wenn Cotta die Preissenkung um den Betrag durchgeführt hätte, welche Dingler vorgeschlagen hat $t^{215}$. Dingler scheint hier auch von der echten Sorge geleitet worden zu sein, daß angesichts der wirtschaftlichen Depression der I82oer Jahre die Okonomen und Cewerbetreibenden, an welche das Journal in erster Linio wande, vom Beru

${ }^{219}$ Siche oben Exkurs: Der Zeitungsplan ${ }^{14}$ Anm. 2 I2 und CA I/22 x5.II. $x 833$. ${ }^{10}$ Schulz: Fachpresse, S. 38 ten würden. Er war es auch, der sich heftig gegen dic I825 von der bayerischen Postadministration eingeführte Einteilung des Landes in Rayons wandtc, welche mit nach der Entfernung vom Versandort steigenden Aufschlägen den Preis de Fernabsatz gefährdeten ${ }^{210}$.

Unbeschadet der wiederholten Dingler'schen Bedenken, hielt aber der Verlag an den $x 6 \mathrm{Al}$ Abgabcpreis fest. Der offensichtlich für seine Zeit hohe Bezugspreis hatte aber den Vorzug, daß trotz des Anstiegs der Herstellungskosten und des Honorars, denen allerdings auch ein Anstieg der Auflagen- und Verkaufsziffern entsprach, der Preis des Polytechnischen Journals über 50 Jahre auf cleicher Höbo gehalten werden kornte In den Bu Briefen Dinglers an Cotta ist namulich erstmals I872 die Rede von der Absicht des Verlages, "infolge der gesteigerten Drucklöhne, Papierpreise etc.« den Preis des Journals auf $2 \mathrm{I} \mathrm{A}$ (xz Taler) zu erhöhen. Emil Dingler erklärtc sein Einverständnis, da er den Preis mit den Preisen der zunächst konkurrierenden technischen Zeitschriften bezüglich Bogenzahl und Figurentafeln in angemessenem Verhältnis stehend fand ${ }^{217}$

6.I.2 Herstellungskosten

Versucht man das Verhältnis des Abgabepreises zu den Herstellungskosten in Verbindung zu setzen, so kann man auf zwei Berechnungen des Verlagshauses Cotta zurückgreifen. Die eine Herstellungskostenrechnung stammt aus dem Jahre 7825 , die andere ist undatiort. Sie dïfte aufgrund der in ihr enthaltenen Angaben über Auflagenzahl und Honorarhöhe jedoch im Jahre $x 850$ erstellt worden sein.

"Kosten d. polyt. J. 182

Druk v[on] I00 Bog[en] 750 Auf[age] aे $f$ Io I000

Druk v[on] I2 Umschlägen

Pap[ier] If Ball[en] aे $f 30$

Schr[ei]bp[apier]

Diese Kostenrechnung ${ }^{218}$ enthält keine Angaben für die Kupfer, fürs Broschieren und für Korrekturen und

20irchner: Zeitschriftenwesen 2 , S. 387.

U11/90 7.To.x872; Dietze: Geschichte, S.7.

CA $1 / 85 a$ Vertrag iüber die Polytechnische Zeitung. nungs-Anmerkung Cottas auf dem Vertragsentwurf danke ich dem freundlichen Entgegenkommen von Frau Dr. Dorothea Kuhn vom Schiller-Nationalmuscum, Cotta-Archiv (Stiftung der Stuttgarter Zeitung) in Marbach/Neckar. führt auch das Honorar nicht auf. Nach der Angabe von Cotta aus der oben zitierten Rechnung an anderer Stell setzte er für Kupferdruck und Papier für 2 Tafeln I8 (Io fl für Kupferdruck, $8 \mathrm{fl}$ für Tafeln) an. Bei $27 \mathrm{im}$ Jahrgang I825 des Polytechnischen Journals erschienenen Kupfertafeln ergibt das 243 f Ausgaben für dic kommt das Herausgeberhonorar in 作 werden, da keine Angaben, nicht berücksichtigt. Addier man diese zusätzlichen Ausgaben zu den oben angefüh ten $x 578 \mathrm{fl}$, ergibt das bei 750 Exemplaren Auflage im Jahr I825 einen Herstellungskostenpreis auf $x$ Exemplar in Höhe von 5,76 Gulden.

Ende des Jahres I825 wurde das Herausgeberhonora auf $4000 \mathrm{fl}$ erhöht. Setzt man nun diese $4000 \mathrm{fl}$ anstelle sich Herstellungskosten für das Exemplar des Polytechnischen Journals im Jahr $I 825$ von 7,76 Gulden.

Die zweite Herstellungskostenrechnung ist, wie gesagt, undatiert und stammt ziemlich sicher aus dem Jah $1850^{210}$.

"Polytechnisches Journal

Ex Satz it. Durck mit 75\% Correctur f $\quad$ I $84 \mathrm{I}, \mathrm{s}$ Satz u. Druck d. Umschl. u. Anzeigen 336,380 Rs. [Ries] Papier à f 35, x2 mit ro\% Aufschlag

Ansatz v. Umschlagpapier $\begin{array}{lr}f & 1337,36 \\ f & 212,15\end{array}$

Stich u. Druck der Tafeln f 705,57

Papier dain f 337,30 f 1043,27

Honorar an Dr. Dingle $5227,3 \mathrm{I}$ \begin{tabular}{cc}
$f$ & $5000,-$ \\
\hline$f$ & I0227,3x
\end{tabular}

Herstellungskosten auf $x E x=f 7,18 x r$

Zwischen den beiden Herstellungskostenrechnungen fallen zunächst die Preisunterschiede bei einzelnen Positionen allem sind die erheblichen Preisdifferenzen von Stich, Druck und Papier der Tafeln zwischen 1825 und 1850 bemerkenswert und in dieser Höhe nicht rech erklärlich. Man kann überdies annehmen, daß die Aufstellung von $\mp 825$ auch deswegen nicht ganz vollständig ist, da sie in schwer lesbarer Konzeptform als Marginalic auf dem Rand eines Briefes, skizzenhaft hingeworfen, entwickelt wurde. Aus diesen beiden Rechnungen ergibt ch aber doch recht deutlich ein Herstellungskostenpreis

${ }^{210} \mathrm{CA}$ II//xo2 Undatiert (vermutlich $\times 850$ ) 
des Polytechnischen Journals zwischen 5,76 und $7,76 \mathrm{~A}$ im Jahr 1825 , der hier allerdings wegen fehlender Einzelangaben tatsächlich vielleicht etwas höher gewesen sein dürfte, und einem eindeutigen Herstellungskostenpreis von 7 Gulden $x 8$ Kreuzer in Jahr I850.

Diese Berechnungen erstrecken sich ausschließlich auf die Herstellungskosten und das Honorar. Sie berücksichtigen nicht die allgemeinen Kosten, wic Handlungsunkosten und Vertriebskosten. Diese waren aber nicht unbeträchtlich. "Kirchner gibt aufgrund Cotta'scher Verlagsarchivalien an, daß das Polytechnische Journal allein für Frachten $400-600 \mathrm{fl}$ erforderte ${ }^{220}$. Zwei Drittel der Auflage, unter anderem auch der Absatz nach Wien22x wurden von Cotts über Leipnig ausgeliefert Bei ber von 370 Exenplaren schloß jedenfalls das Polytechnische Journal noch mit Verlust ab. Erst 1824 wurde bei cinem Absatz vori 5 I2 Exemplaren die Rechnung für das Polytechnische Journal bei Cotta erstmals nachweislich mit Gewinn abgeschlossen ${ }^{222}$. Der Gewinn scheint sich allerdings mit dem steigenden $\mathrm{Ab}$ satz trotz der Honorarerhöhung von Ende 8825 rasch gesteigert zu haben. Kirchner gibt an, das Polytechnische Journal perbrachte 5 Jahre nach seiner Grïndung bereis

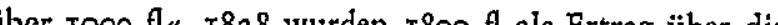
(1) 1000 Alc 1828 usgewis Journal ist damit nach den ersten schwicrigen Erscheinungsjahren für das Verlagshaus Cotta bald ein finanzicll recht ergiebiges Unternehmen geworden.

220 Kirchner: Zeitschriftenwesen 2, S. 474. Auf S. 47x druckt Kirchner eine Kostenrechnung des Polytechnischen Journals in
Prozentrelationen für $x 8 x 9$ und 1828 ab. Diese Kostenrechnung kann zu den oben angeführten Berechnungen als Ergänzung herangezogen werden, doch leidet die Vergleichbarkeit dieser Kostenrechnung an unterschiedlichen Aufschlïssclungen und an particllen Lü̈cken in den Angaben.

${ }^{221} \mathrm{~J}$. G. Dingler mokierte sich wiederholt über das beim Absatz nach Wien deutlich hervortretende "unbegreifliche Spcdiionsysstemw. " Jetzt führt man die Waare über Leipzig spazicren bei 4 bis 6 fach höherer Fracht und 8 bis ro fachen Zeit-

Dingler plädierte beim Absatz nach Wien für die Floßsverund im Winter für die L andfrect äber Sontur.

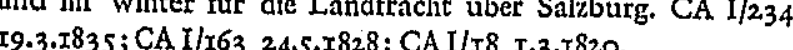

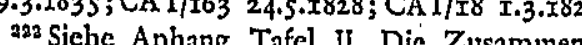

afel danke ich wiederum. Frau $\mathrm{Dr}$. Dorothen $\mathrm{K}$. Schiller National-Muscum, Cotta-Archiv, Marbach/Neckar. Diese Angaben aus den Druckauftragsbüchern finden sich cbenfalls bei: Hamann: Zeitschriften, Anhang Tabelle Ir. Einzelne Angaben dicser Druckauttragsbücher-Tabelle differiere mit Auflagenziffern bei Kirchner: Zeitschriftenwesen 2, S. 46r,

\subsection{Auflage und Absatz}

Entgegen der wiederholten optimistischen Erwartung von J. G. Dingler, daß Cotta bei dem Polytechnischen Journal "auf einen bedeutenden Verschleiß zählen« könne oder, daß der "Absatz unbezweifelte sei, ließ sich der Absatz des Journals zunächst nicht sehr erfreulich an a $^{223}$. Die J. G. Cotta'sche Buchhandlung scheint sich auch zunächst im unklaren wegen der auszudruckenden Auflagenhöhe gewesen zu sein. Nach einer Anfrage an J. G. Dingler, wie hoch dic Auflagenzahlen seiner bisherigen Veröffentlichungen gewesen seien, und der Antwort Dinglers, daß von seinem Journal für die Zitz-, Kattunoder Indiennedruckerei (Augsburg, Leipzig I806-07) xooo Exemplare aufgelegt worden seien und überdics cine zweite Auflage notwendig geween Journals die gleiche Zahl zugrunde224. Vom Jahrgang I820 des Polytechnischen Journals wurden IoIo Exemplare aufgelegt. Abgesetzt wurden aber im Jahr $x 820$ nur 337 Exemplare.

Das erste Heft des Jahrgangs I82I wurde noch mit I000 Exemplaren, die weiteren Hiffte jedoch nur noch mit 750 Exemplaren aufgelegt. Der Absatz betrug I82I II5 und I822 - wie gesagt - 370 Exemplse. Alle dre Alle drei Jahrgänge des Polytechnischen Journals I820-I822 schlossen mit Verlust für den Verlag ab $\mathrm{b}^{222}$

Wegen dieser wenig erfreulichen Absatzentwicklung setzten von Anfang an Bemühungen zur Absatzsteigerung des Polytechnischen Journals ein. Die Abhilfemaßnahmen zielten in zwei. Richtungen. Erstens suchte man Anlehnung an die staatliche oder halbstaatliche Gewerbeförderung bzw. den Staat, der den nachgeordneten technischen Behörden das Poly denishe Jour an Anschaffung vorschlagen sollte. Zweitens hoffte man, durch allgemeine Werbung in Zeitschriften und Zeitungen den Bèkanntheitsgrad des Polytechnischen Journals und damit den Absatz zu steigern.

\subsection{Einschaltung der Behörden zur}

\section{Absatzsteigerung}

Es ist unbekannt, ob die am Anfang des 3. Bandes abgedruckte Widmung des Herausgebers im ersten Jahrgan des Polytechnischen' Journals an den bayerischen König, den »erhabenen Beschüzer aller gemeinnüzigen Künster,

${ }^{223} \mathrm{CA}$ I/II 27.II.I8I9; CA I/2I 4.4.I820; Dietze: Geschichte, ${ }_{224}^{4}$ CA I/xo 3.Ix.I8I9. einen sichtbaren Erfolg hatte. Diese Widmung schadete jedoch auch sicherlich nicht den bald einsetzenden Kontaktaufnahmen mit den zuständigen Regierungsstelle über die Absatzverbesserung. So schrieb Dingler I82 an Cotta: "Ehen so werde ich dieser Tago mit unserem ancomal gehen ist, daß unsere Regierung, die das Erscheinen dieses Journals sehr wohlgefällig aufnahm, die technischen Stellen für die Anschaffung und Bexechnung anweist, wodurch ein bedeutenderer Absatz bezweckt würde«"225.

Zunächst erfolgreicher als in Bayern, das im Kunstund Gewerbeblatt des polytechnischen Voreins ohnehin

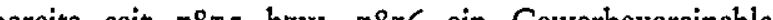
esaß, war Dingler mit seinen gezielten Absatzsteigeungsbemühungen in Württemberg und Preußen. $x 82$ schrieb er Cotta, daß er zum besseren Absatz des Polytechnischen Journals dem König von Württemberg ein Exemplar mit der Bitte geschickt habe, "dasselbe prüfen zu lassen und wenn es der Auszeichnung werth geachtet würde, es den technischen Behörden $z u$ empfehlen«. Im 7. Band des Journals konnte er dem Publikum den Erfolg seiner Bemühungen mitteilen, daß der König von Württemberg geruht habe, sin Rücksicht der Nüzlichkeit, welche das polytechnische Journal für den deutschen Kunst- und Gewerbefleiß hat ... dasselbe durch das Departement des Innern den Behörden empfehlen zu lassen«. Intern bat Dingler Cotta, aus dieser Empfehlung seiner Stuttgarter Regierung den geeigneten Vorteil zu ziehen.

Von der preußischen Regierung versprach sich Dingle noch einen »brilantern Erfolg für den Absaz«. Auch hie konnte er dann dem Publikum mitteilen, daß der Köni von Preußen dem Herausgeber eröffnet habe, »daß das Staatsministerium des Handels und der Gewerbe beauftragt sey, genantes Journal zu prüfen, um über die Empfehlung desselben an die technischen Behörden das Wetere zu verfügen $\aleph^{220}$.

In Bayern ergab sich eine große Chance für die Absatzsteigerung in den Jahren $1825 / 26$. Durch eine Erläuterung zum "Gewerbsgesetz « sollten damals in Bayern dic Zünfte gleicher Gcwerbe zusammengeschlossen und angehalten worden, "sich durch Schriften auszubildenu. Aus diesem Anlaß hatte Dingler von München die "Versicherung " erhalten, daß diesen "demnächst zu bildenden Gewerbsvereinen die Haltung des polytechnischen Journals von der allerhöchsten Stelle amtlich empfohlen ${ }^{225} \mathrm{CAI} / 32$ 9.5.x821; Dietze: Geschichtc, S. 4.
${ }^{220} \mathrm{CA} \mathrm{C} / 4 \mathrm{I}$ 30.r.I822; Polyt. Journal 7 (I822), S. 254 . wird «227. Nachdem Dingler nocl beim Ministerium wegen dieser Empfehlung urgiert und er auch den Baron von Cotta gebeten hatte, beim bayerischen König wegen dieser Empfehlung vorstellig zu werden, kam man mit den staatlichen Stellen sin betreff des Absazes resp, der des Regelune Allen der Regelung: Allen, etwa rooo Behörden in Bayern ("Magistrate, Landgerichte, Herrschaftsgerichte, Rentämter ec.«) sollte ein Gratisexemplar des Polytechnischen Journals mit einem Inhalt der vorhergegangenen Hefte und einer "gedruckten Erläuterung über Sclbst-Anschaffung und Weiterempfehlung « zugesandt werden. An größere Städte und Landgerichte sollten zwei Exemplare gesendet werden. Dingler gefiel diese Regelung, und er heffe, außerhalb Bayerns anwenden zu können. Bei dem zum Versand kommenden Heft wollte cr größte thematische Mannigfaltigkeit erreichen'228.

Leider tauchen in den Briefen Dinglers an Cotta keine exakten Zahlen auf über die tatsächlichen Absatzsteigerungen aufgrund dieser amtlichen Empfehlungen. Die Angaben über dic Auflagenhöhe und den Absatz sind ebenfalls zu lïckenhaft um deutliche Sprünge in Auf-

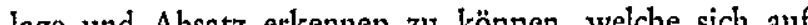
diese Empfehlungen zurückführen lassen können. Die Notwendigkeit der Anlehnung der freien technischen Berichterstattung an die amtliche Gewerbeförderung zeigt aber doch, daß in diesen $x 820 e r$ Jahren der eigentlich durch die Zeitschrift angesprochene Leserkreis der an technischen Fortschritten interessierten Gewerbetreibenden entweder noch nicht vorhanden oder daß er zu dünn war, um einem Blatt von der Art des Polytechnischen Journals einen hinreichenden Absatz außerhalb des Berciches der staatlichen oder halbstaatlichen Wirtschaftserziehung sichern zu können. Die Mitgliederliste des Polytechnischen Vereins für das Königreich Bayern von $I 8 \mathrm{r} 6$ bis $\mathrm{I} 824$ mit nur $30 \%$ Gewerbetreibenden und Freiberuflichen und $70 \% \mathrm{im}$ Staatsdienst stehenden Mitgliedern sowie dic Mitgliederzusammensetzung des Vereins zur Beförderung des Gewerbefleißes in Preußen in seinem Grïdungite 2822 bei dem 2 To yon 367 Mitgliedern Beamte waren, sind weitere Hinweise darauf, daß in der Ubergangsphase vom Kameralismus zum Liberalismus zunächst das Wirtschaftsleben und die $\mathrm{Ge}$ werbeförderung noch außerordentlich stark von der

CAI/9x 23.rr.x825; CAI/ror 16.2.I826.

CAl/2 28.7825 CA I/TO2 I0.4. $\times 826$. 
staatlichen Intervention und Bevormundung bestimmt blieben ${ }^{220}$.

Das Ziel der Absatzförderung durch amtliche Kaufempfehlungen an die Dienststellen ist ohne Zweifel erleichtert worden durch dic Einflußmöglichkeiten und das Ansehen, das Baron Cotta und in gewissem Grade auch Ansehen, das Baron Cotta und in gewissem Grade auch der Herausgeber bei den staatlichen Stellen besaßen. Für
den weiteren Absatz hatte aber diese Einschaltung der Behörden den Vorteil, daß damit das Polytechnische Journal schon in den $x 820$ er Jahren einen festen krisensicheren Abonnentenkreis bei Schul-, Amts- und Dienststellenbüchereien fand, ehe überhaupt erst weitere freie berufstechnische Blätter erschienen waren denen damit dieser Absatzweg erschwert, wenn nicht yerbaut war.

\subsubsection{Werbung in Zeitschriften}

und Zeitungen

Gegen die Verminderung der Druckauflage nach dem crsten Heft des zweiten Jahrgangs im Jahre I82I hatte sich J. G Dingler mit dem Arsument außer den Anzige WectyChnisch JourtaVerlags bisher noch keine Werbung für das Polytechnische Journal in anderen Blättern betrieben worden sei. Der Herausgeber wollte es daher unternehmen, daß in süddeutschen Zeitungen und Zeitschriften die Rede vom Polytechnischen Journal sein sollte, während der Verlag für Anzeigen in einigen nördlichen Blättern Deutschlands sorgen solite. Dingler meinte auch, daß eine Herabsetzung des Abgabepreses des Joumals dem Abstr angute rom Morgentatt fuir die Morgenblatt fur die gebildeten Stände des Cotta-Verlags m nördlichen Deutschland nach Ansicht Dinglers zu wenig gelesen würden, sollte vor allem der Inhalt der Hefte des Journals in verschiedenen Zeitungen Norddeutschlands angezeigt werden. Neben dem Allgemeinen Anzeiger der Deutschen und dor Hamburger Zeitung gab er dabei auch die Wiener Zeitung, den Nürnberger Correspondent, da dieser in Sachsen viel gelcsen worde, und die Straßburger Zeitung als geeignet für Inserate an

I826 führte Dingler in einer dem Verlag übersandten Liste wiederum diejenigen Zeitungen auf, in welchen für das Polytechnische Journal geworben werden sollte. Au ihr werden zum Teil die Regionen sichtbar, in welche

${ }^{220}$ Troitzsch: Entwicklung, S. 334f.; Schnabel: Deutsche ${ }_{230}$ CAte 3, S. 33T; Schulz: Fachpresse, S. 33 ${ }_{202} \mathrm{CAI} / 37$ 2.5.II. 182. man offensichtlich den Verschleiß für verbesscrungswürdig hielt. Ein Avertissement sollte an Perthes \& Besser in Hamburg geschickt werden, ferner sollte inseriert werNürnberger Correspondenten, dem Schwäbischen Merkur, der Straßburger Zeitung, einer der gelesenen Schweizer Zeitungen, der Wiener Hofzeitung, der Prager Zeitung und im Brockhaus Anzeiger ${ }^{232}$

Die meisten Anzeigen für das Polytechnische Journal erfolgten jedoch in den verlagseigenen Zeitungen und Zeitschriften des Cotta-Verlags, so im Intelligenzblatt den landwirtschaftlichen Blättern sowie in den anderen "verschiedenen Zeitschriften des Verlags «"233.

Vom Abdruck der durch viele gemeinnützige Abhandlungen ausgezeichncten Inhaltsverzcichnisse der letzten Hefte in der Allgemeinen Zeitung versprach sich Dingle einmal gar einen "Zugang von too neuen Abonnenten ${ }^{234}$. Zeitweilige Sperren, die vom Verlag Cotta bezüglich der Werbung verlagseigener Werke in der Beilage der Allgemeinen Zeitung vorhängt wurden, suchte Dingler durch Ausnahmegenehmigung für das Polytechnische Journal zu umgehen ${ }^{234}$. Fast alle diese Angaben über Wcrbcaktionen beziehen sich auf die $1820 e r$ Jahre. Als um die Mitte der I840er Jahre der Absatz, nach den Zahlen der Druckauflagen zu schließen, nicht recht vorankam, meinte J. G. Dingler in einem Schreiben an Cotta, daß die Inhaltsanzeigen des Polytechnischen Journals wiederum "wenigtens ein paar Mat in nordische monatlic . 1835 hat der Herausgeber den Absatz nach Rußland steigern wollen. Er hofftc auch, nach Amerika gute Geschäfte machen zu können und fragte bei Cotta an, ob er denn dorthin keine solide Verbindung habe ${ }^{230}$

Bei den Inseraten in den Zeitschriften und Zeitungen kam die Hauptwerbefunktion dem Abdruck der Inhalts verzeichnisse der letzten Hefte oder des letzen Hefes Jounil Dingler äußerto sich einmal über die Art einer Annonce zur Absatzsteigerung: »Was aber hauptsächlich zur Vermehrung de Abonnenten vermittelst Annonce beytragen dürfte, is meiner Meinung nach einzig \& Allein die Verbreitun der Inhalts-Verzeichnisse, da dic Mannigfaltigkeit der

\section{${ }^{202} \mathrm{CA}$ I/100 28.x.7826.}

${ }^{2033} \mathrm{CAI} / \mathrm{IS5} 5 \mathrm{I} .2 . \mathrm{I828} ; \mathrm{CAI} / 204$ 23.2.x8

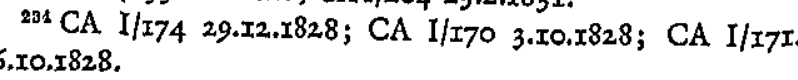

${ }_{200}^{200} \mathrm{CAII} / 99$ undatiert (wohl 1846$)$.

${ }^{230} \mathrm{CA} \times / 23524.5 . \times 835$
Gegenstände fast jedem Industriellen etwas bieten $\mathrm{muß} \ll^{235}$.

Neben dem Abdruck der Inhaltsverzeichnisse wurde

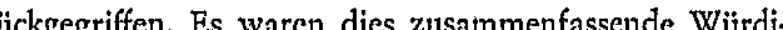
gungen des Polytechnischen Journals, die auf den ersten Blick leicht den Eindruck erwecken konnten, als ob es sich um eine unabhängige Besprechung des Journals von dritter Seite und nicht um einen Werbetext des Verlages oder der Redaktion handelte ${ }^{237}$.

Diese verschiedenartigen Bemühungen um die Steigerung des Absatzes machten im Verein mit dem Inhalt das Polytechnische Journal binnen kurzem zu einem recht erfolgreichen Blatt. Dem nachweislich ersten $A b$ schluß mit Gewinn im Jahr $x 824$ folgten nun gesteigerte schluß mit Gewinn im Jahr 1824 folgten nun gesteigerte
Auflagen- und Absatzzahlen. 1826 betrug die Auflagenhöhe rooo Exemplare, von denen 780/810 abgesetzt wurden. I834 wurden I250 Exemplare aufgelegt und Ior6 verkauft.

Dor publizistische Erfolg des Polytechnischen Journals machte sich jetzt auch darin bemerkbar, daß die Verlagshandung dazu wberech

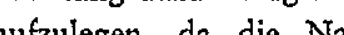
aufzulegen, da die Nachbestellangen dazu zwangen. Bereits 1833 hatte J. G. Dingler wegen der vielen Anfragen nach fehlenden Bänden des Polytechnischen Journals angeregt, diese Bände nachzudrucken und sie gegebenenfalls zur Gewinnung wciterer Abnchmer um einen ermäßigten Preis abzugeben ${ }^{238}$. Im Werbetext für das Polytechnische Journal im Börsenblatt 1834 wurde ebenfalls die Notwendigkeit des Nachdrucks vergriffener bän enaue Zeitpunkt läßt sich nicht angeben, aber um dic Mitte der I830er Jahre muß dann der Nachdruck der
vergriffenen Bände des Polytechnischen Journals in Gang gekommen sein.

Druckauflage und Absatz stiegen in den nächsten Jahren weiter an. 1840 betrug die Druckauflage im Zeitraum Jänner-Juni 5500 und von Juli-Dezember Z450 Stïck. 846 legte man die ersten zwei Hefte erstin 2000 Exemphe me in 2000 Uberzoger war, denn ab x. Juli ging man gleich um 400 Exemplare aul 1600 Sack Auflage auf $x_{400}$ Exemplare reduziert. In den weiteren I85oer Jahren schnellten jedoch im Gefolge der damals einsetzenden - wirtschaftlichen Aufstiegswelle, welche wirtschaft einband, die Auflagen- und Absatzzahlen

$\mathrm{Zu}$ dieser Gattung gehörte $\mathrm{z} . \mathrm{B}$. dic Anzeige im Börsen-

blatt $x,(1834)$, Sp. $27 x-273$ deutlich in die Höhe. 1854 , ein Jahr yor J. G. Dinglers Tod, wurden I724 Exemplare (nohne die 27 Freiexemplare (1) abgesetzt ${ }^{230}$. 1855 wurden im I. Heft 2400 und ab dem 2. Heft 2000 Exemplare des Polytechnischen Journals aufgelegt. I858 betrug dic Auflage im I. Helt erstmals 3000 Exemplare, mußte aber dann im 2, und 3 Heft auf 2600 und ab dem 5 . Heft auf 2400 Exemplar zurückgesteckt werden. Immerhin bleibt von $x 850$ bi x858 die Druckauflagenerhöhung um I000 bzw. $x 600$ Exemplare bemerkenswert. Für die folgenden Jahre sind keine weiteren Auflagenziffern bekannt. Doch hatte sich x86I der Absatz sogar geringfügig verringert, stieg abe dann seit dem Jahr 862 in welchem allein 80 Exemplare mchr abgesetzt worden waren, wieder an ${ }^{240}$.

Mit seinen Auflagenzahlen gehörte das Polytechnische Journal zur Spitzengruppe der erfolgreichen Cotta'schen Zeitschriften und rangierte zum Teil auch weit vor de Auflagenzahlen vergleichbarer technischer und gewerblicher Zeitschriften. So lag die Druckauflage der seit $x 8_{3}$ bei Cotta erscheinenden allgemeinwissenschaftlichen Zeitschrift Deutsche Vierteljahresschrift im Jahr 2847 bei rooo Exemplaren. Das Polytechnische Centralblat in Leipig und dic Berg- und hïtrenmännische Zeitun in Lipzis und dic Verendlungen des Vereins zur Beförderun des Gewerbefleißes in Preußen und die des Kunst- und Gewerbeblattes des polytechnischen Vereins in Bayern betrug $x 847$ tooo bzw. x200 Exemplate. Hingegen wurden vom Polytechnischen Journal I847 I600 rcsp. ab Juni dieses Jahres I550 Exemplare ausgedruckt, eine Zahl, die in dicsem Jahr nur von den großherzoglich hessischen Gewerbevereinsblättern (2roo) und der All(20) Ver von Teilen der Auflagen zeigen überdeutlich, daß das Polytechnische Journal ein außergewöhnlich erfolgreiches Publikationsorgan wa

Leider versagt das Quellenmaterial völlig bei der $\mathrm{Fra}$ ge nach der regionalen Absatzstreuung und dem Abonnenten- und Leserkreis des Polytechnischen Journals. Aufgrund der wiederholten Werbebemühungen in Norddeutchland ist anzunelmen daß der Absatz in Süddeutschland größer als im Norden war242. Gelegentliche

${ }^{230} \mathrm{CA} I I / 35$ Ix.2.x855

${ }^{240} \mathrm{CA} \mathrm{II} / 57$ 5.2.2.8862; CA II $/ 60$ 8.4.1862; CA II/70 5.2.1865. ${ }_{241}$ Ubersichten über dic Druckaullagen im Jahr I847 sieh bei Hamann: Zeitschriften, Anhang Tabelle II und I3.

${ }_{242} \mathrm{CA} I I / 44$ 3x.x.r858, schrieb Emil Dingler an Cotta: "Das relative Verhältnis des Absatzes des polytechnischen Journals in Nord- und Süddeutschland, 
Außerungen, daß das Journal in England "gekannt istu, setzung des Mitarbeiterkreises auf die regionale Her$\mathrm{da} ß$ es in Holland geschätzt werde, daß einige Exemplare auch nach Rußland abgesetzt wurden oder daß es das "gelesenste" in Deutschland sei, helfen in dieser Frage kaum weiter243. Auch Rückschlüsse von der Zusammen-

24s Polytechnisches Journal 27 (18828), S. xo5; 35 (x830), S. 70; $60(x 836)$, S. $x 66 ; \mathrm{CAI} / 235$ 24. 3. 1835 kunft und die berufliche Zusammensctzung des Leserkreises können eine Leseranalyse nicht ersetzen. Sic wären im Ergebnis zu oberflächlich. Dicse Untersuchung über das Polytechnische Journal im Zeitraum $x 820$ bis x855 muß daher damit schließen, daß sie quellenbedingt die Antwort auf die Frage nach dem Leserkreis und der publizistischen Wirksamkeit dieser Zeitschrift offenläßt.

Tafel I

7 Anhang

Die Herkunft der Aufsätze im Beitragsteil des Polytechnischen Journals nach den Erscheinungsländern der ausgewerteten Zeitschriften (einschließlich Monographien und Originalbeiträgen)

Zand:

Erscheinungsland

der Beiträge:

$\begin{array}{llllllllllllllllll}\text { Deutscher Bund } & \text { I3 } & \text { xo } & \text { ro } & 4 & 3 & 5 & 6 & 2 & \text { I5 } & \text { I3 } & 23 & 29 & 24 & \text { I9 } & 25 & 31 & 39\end{array}$

$\begin{array}{llllllllllllllllll}\text { Orig.art") I2 } & 9 & 9 & 4 & 3 & 5 & 4 & 2 & \text { II } & 2 & 7 & 3 & \text { I4 } & 6 & \text { IO } & \text { II } & \text { I6 } \\ \text { Zss.art. } & \text { I2 } & & & \end{array}$

4 II $I 6 \quad 26$ IO $x_{3}$ IS $20 \quad 23$

England

Frankreich

Italien

Belgien

Holland

Schweden

USA

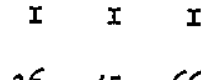

$\begin{array}{rrrrrrrrrrrrrrrrr}26 & 45 & 66 & 73 & \text { IOI } & 70 & 46 & 54 & 46 & 55 & 56 & 47 & 43 & 29 & 34 & 44 & 35 \\ 5 & x_{2} & \text { I2 } & 21 & 28 & 30 & 28 & 34 & 24 & 24 & 3 x & \text { I9 } & 24 & 3 x & 38 & 35 & 4 x\end{array}$

Miszellen

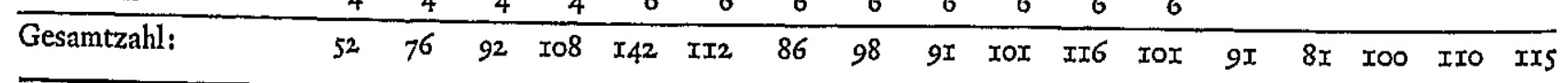$$
3
$$$$
44
$$$$
\text { I }
$$

I

I $I$

* Dem Deutschen Bund wurden bei den Originalartikeln auch die wenigen deutschsprachigen Originalbeitrïge von Baltenveröffentlichung im Polytechnischeizern oder Schweden zugerechnet, da ja das Ersterscheinungsland dieser Artikel durch ihre

\section{Tafel II}

Auflage und Absatz von»Dinglers Polytechnisches Journal« (Auszüge aus den Cotta'schen Druckauftragsbüchern)

Jahrgang Auflagenhöhe Absatz Anmerkungen Jahrgang Auflagenhöhe Absatz Anmerkungen

\begin{tabular}{|c|c|c|c|c|c|c|}
\hline 1820 & roro & 337 & Abschluß mit Verlust & I 826 & I000 & 780/8 xo Abschluß mit Gewinn \\
\hline I82I & & & & I827 & - & 890 Abschluß mit Gewinn \\
\hline x. Heft & 1000 & 3 I 5 & Abschluß mit Verlust & I828 & xroo & 880/920 Abschluß mit Gewinn \\
\hline ab 2. Heft & 750 & - & - & $r 829$ & - & - \\
\hline I822 & - & 370 & Abschluß mit Verlust & $x 830$ & I200 & 920 Abschluß mit Gewinn \\
\hline I823 & - & - & - . & I83 & Ixoo & 983 \\
\hline 1824 & - & $5 \pm 2$ & Abschluß mit Gewinn & 1832 & - & - \\
\hline 1825 & - & - & - & & & \\
\hline
\end{tabular}

\begin{tabular}{|c|c|c|c|c|c|c|c|}
\hline Jahrgang & $\begin{array}{l}\text { Auflagen- } \\
\text { höhe }\end{array}$ & Absatz & Anmerkungen & Jahrgang & $\begin{array}{l}\text { Auflagen- } \\
\text { höhe }\end{array}$ & Absatz & Anmerkungen \\
\hline I833 & - & - & - & 3. Heft & $I 800$ & - & - \\
\hline \multirow[t]{4}{*}{$x 834$} & x250 & ausge- & & $a b$ r. Juli & $x 600$ & - & - \\
\hline & & liefcrt & - & $I 847$ & $x 600$ & - & - \\
\hline & & Ostern & & ab Juni & x550 & - & - \\
\hline & & I0I6 & & $x 848$ & I550 & - & - \\
\hline I 835 & $x 35^{\circ}$ & - & - & I849 & I500 & - & - \\
\hline I 836 & $x_{400}$ & - & - & ab April & I 400 & - & - \\
\hline 1837 & & & & I850 & $x 400$ & - & - \\
\hline Jan./Febr. & I500 & - & - & $x 85 x-1854$ & - & - & - \\
\hline März-Sept. & $I 450$ & - & - & $x 854$ & - & $x 724$ & (nach CA II/35 \\
\hline Okt.-Dez. & 1400 & - & - & & & & II. 2. $x 855$ ) \\
\hline 1838 & I450 & - & - & $x 855$ & & & \\
\hline I839 & I500 & - & - & I. Heft & 2400 & - & - \\
\hline$I 840$ & & & & ab 2. Heft & 2000 & & - \\
\hline Jan.-Juni & $x_{500}$ & - & _ & I856 & & & \\
\hline Juli-Dez. & I 450 & - & - & I. Heft & 2400 & - & - \\
\hline$I 84 x$ & I 450 & - & - & 2. u. 3. Heft & 2000 & - & - \\
\hline$I 842$ & I500 & - & - & $\mathrm{ab}$ 4. Heft & 2100 & - & - \\
\hline$I 843$ & I500 & - & - & $x 857$ & & & \\
\hline I844 & & & & x. u. 2. Heft & 2500 & - & - \\
\hline Jan.-Juni & I500 & - & - & ab 3. Heft & 2300 & - & - \\
\hline Juli-Dez. & $I_{450}$ & - & - & $x 858$ & & & \\
\hline 1845 & x500 & - & - & I. Heft & 3000 & - & - \\
\hline I846 & & & & 2. u. 3. Heft & 2600 & - & - \\
\hline 2 Hefte & 2000 & - & - & ab 5. Heft & 2400 & - & - \\
\hline
\end{tabular}

Quellen

8 Quellen- und Literaturverzeichnis
Polytechnisches Journal. Hrsg. von Johann Gottfricd Dingler

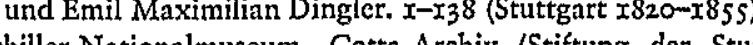
garter Zeitung). Marbach/Necka

26I Briefe Johann Gottfried Dinglers (17778-x855) an die I848. Zitiert als: $C A$ I/Nummer des Briefes und Datum. I02 Briefe Emil Maximilian Dinglers (x806-I874) an die Cotta'sche Verlagsbuchhandlung aus dem Zeitraum I8291874. Zitiert als: CA II/Nummer des Briefes und Datum.

\section{Literatur}

Allgemeine deutsche Real-Encyklopädie für die gebildeten Stände. Conversations-Lexikon I2.I4 (Leipzig: Brockhaus ${ }^{10} \times 854$ ).

ALLIVANG, Alexander: Augsburger Straßennamen - Dinglerstraße. Alt-Augsburg, Jg. X, H. 4/5 (I937), S. 72-78.

Börsenblatt für den Deutschen Buchhandel und für die mit ihm verwandten Geschäftszwcige, Jg. I (Leipzig I834), Sp. 27x273.
Conversations-Lexikon oder encyklopädisches Handwörterbuch für gebildete Stände 9 (Leipzig, Altenburg: Brockhaus

I8X77.
DIETZE, W.: Aus der Geschichte des Journals. Dinglers Polytechnisches Journal I820-T2020. Festschrift zum hunderjährigen Bestehen der Zeitschrift (Berlin I920). S. 3-8.

DINGLER, Emil: Nekrolog auf Johann Gottfried Dingler. Polytechnisches Journal I38 (x855), S. 396-400.

DINGLER, Helmuth: Johann Gottfried Dingler. Ncue Deutsche Biographie 3 (I957), S. 730.

EBERLE, Josef: Johann Friedrich Cotta. Sonderdruck aus der Stuttgarter Zeitung (1952).

FiLL, Karl: Einfuhrung in das Wesen der Dezimallklassifikation (Berlin, Köln, Frankfurt/M. ${ }^{3}$ I969).

Das große Conversations-Lexikon fur die gebildeten Stande Hrsg. von Josef Meyer, 4x (Hilldburghausen, Amsterdam usw. I85x).

HAMANN, Hans-Joachim: Die ökonomischen und technischen Zeitschriften in der Frühzeit der deutschen Industrie. Mschr. Univ. Köln (Köln I957/58). 
KARMARSCH, Karl: Kritische Ubersicht der deutschen technologischen Journalistik. Polyt. Journal Bd 73 (I839), S. 208234, 299-3IX, 370-395; Bd 76 ( $(x 840)$, S. 52-7I, I2 8-r 52 Bd 78 (I840), S. 297-313, S. 369-400; Bd 83 (I8 42 ), S. 53-83 S. I 4 8- $x 63$, S. 22I-240, S. $286-338$.

-,: Geschichte der Technologie seit der Mitte des 18. Jahrhunderts (München 8872 ).

J Nkrolog aut Emil Maximilian Dingler. Polytechnische

: Johann Gottricd Dingler. Allgemeine Deutsche Biographie 5 (I877), S. $239 \mathrm{f}$.

-,: Ein Lebensbild. Gezeichnet nach dessen hinterlassenen Ergañzungen von Egb. Hoyer (Hannover 1880 ).

KIRCHNER, Joachim: Das deutsche Zeitschriftenwescn. Scin Geschichte und seine Probleme I. 2. (Wiesbaden $x 958$, I962). KLemM, Friedrich: Technik. Eine Geschichte ihrer Problem (München I954).

KrIEG, Walter: Materialien zu einer Entwicklungsgeschicht der Bücher-Preise und des Autoren-Honorars vom Is. bis zum 20. Jahrhundert (Wien, Bad Bocklet, Zürich I953).

LoHRRR, Lisclotte: Cotta. Geschichte eines Verlages I659I959 (Stuttgart 1959).

-,- (Bearb.): Schiller-Nationalmuseum Marbach/Neckar. Bestandsverzeichnis des Cotta-Archivs (Stiftung der Stuttgarte Zeitung) I: Dichter und Schriftsteller (Veröffentlichungen

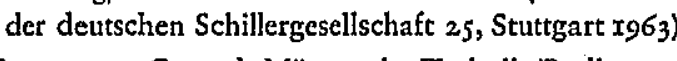
Matschoss, Conrad: Männer der Technik (Berlin I925). MrNZ, Gerhard: Die Zeitschrift. Ihre Entwicklung und ihre Lebensuedingungen. Eine wirtschaftsgeschichtliche Studie (Stuttgart I928).

POGGENDORFF, Johann Christian: Biographisch-literarisches Handwörterbuch zur Geschichte der exakten Wissenschaften $x-5,2$ (Leipzig I863-I926).

FFLe, Albert: Cotta (Geisteshelden I8, Berlin I895).

CHirLer, Herbert: Georg Cotta. Schwäbische Lebensbilder 2 (Stuttgart x94I), S. 54-66.

Cotta. Schwäbische Lebensbilder 3 (Stuttgart I942), S. 72- 224

CHNABEL, Franz: Deutsche Geschichte im neunzehnten JahrBreisgau I934)

SchuLTes, Joseph August: Nekrolog. Polytechnisches Journal 42 (I83I), S. 222-232.

SCHULTRS, Julius Hermann: Nekrolog. Polyt. Journal 78 (x840). S. 77-80.

SchuLZ, Kurt: Um dic Zukunft der technischen Fachpresse. Entwicklung und Gegenwartsprobleme der Berichterstattung in Deutschland (Berlin 193I).

TMM, Albrecht: Kleine Geschichte der Technologie (Urban Bücher 78, Stuttgart 2964$)$.

(Uni-TaschenWillolin: Dingers

Tournal - eine I970), S. X-I2.

1970), S. X-I2. Zeitschriften in Deutschland zwischen $x 820$ und $x 850$ Wissenschaft, Wirtschaft und Technik, Studien zur Geschichte. Wilhelm Treue zum 6o. Geburtstag. Hrsg. von Karl-Heinz Manegold (München 1969, S. 33I-339).

ZORN, Wolfgang: Augsburg. Geschichte einer deutschen Stadt (Augsburg I955).

\section{Zusammenfassung}

Das I820 gegründete "Polytechnische Journal" ist die alteste technische Zeitschrift Deutschlands, welche ohne Verbindung zu einem Gewerbevercin cntstanden ist. Ih Gründer und erster Herausgeber war der Augsburger Chemiker und Fabrikant Johann Gottfried Dingler ( $x 788$ bis 2855 ). Verlegt wurde die Zeitschrift von dem be kannten "Klassiker-Verlag" J. G. Cotta in Stuttgart.

Nach einer allgemeinen Einführung in die Situatio der technischen Berichterstattung am Beginn des Ig. Jahrlhunderts in Deutschland werden in der vorlicgenden Studie zunächst untersucht die Gründung, das Programm und die Personen der Herausgeber des Polytechnische Journals. Fragen der Herstellung der Erscheinungsweis und des Erscheinungsbildes sowic antsprechend de Entwicklung der Technik und der Industrie wurde der Nachrichtenstoff in erster Linie englischen, dann französischen und nur sehr allmählich ansteigend deutschen Zeitschriften entnommen. Das Polytechnische Journal war vorrangig ein Refcrateorgan. Der Anteil der Orig- nalbeiträge blieb stets gering. Nach anfänglich schwachem Absatz ( 8820337 verkaufte Exemplare) und $\mathrm{Ab}$ schlüssen mit Verlusten wuchs das Polytechnische JourDeutschands heron (1854 1724 verkaufte Exemplarc) Grundlage des Erfolgs waren ein breiter, nahezu alle Gewcrbezweige abdeckender "polytechnischer" Inhal inc gemeinverständliche, durch Abbildungen veralschaulichte Stoffdarbietung und cinc erstaunliche Schnelligkeit in der Verbreitung der neuesten Erfindungen des In- und Auslandes. Das Polytechnische Journal leistete in der Frïhindustrialisierung einen atholichen Beitzes zur Erzichung der deutschen Gewerbetreibenden zur Industrie. Die Entwicklung des Polytechnischen Journals wurde bis zum Jahre I855 untersucht. Durch das Entstehen fachlich und berufsständisch stärker spezialisierte Zeitschriften endete um dic Mitte des I9. Jahrhunderts Charakters. die eigentliche Blätezeit der Zeitschriften polytechnischen
The 'Polytechnische Journal', founded in 1820 , is the oldest technical periodical in Germany, which came into cxistence without having any prior connection with business or trade association. Its founder and firs Johnin Gottried Dinglee (7778-1855), chemist and factory owner of Augsburg. The periodical was published by the well-known 'publisher of classics' J. G. Cotta in Stuttgart

Following a general introduction into the situation o technical reporting at the beginning of the xgth century in Germany, the present study proceeds to examine the foundation, the programm and personalities of the editors of this 'Polytechnische Journal'. Subsequently, problems dealing with production, frequency of publication, general appearence, as well as the editor's cation, general appearence, as well as the editor's
share of the royalties are topics under discussion. In line share of the royalties are topics under discussion. In line
with the development of technology and industry, topical with the development of technology and industry, topica primarily from English, later from French, and only very gradually from German periodicals. Dingler's Journa was primarily a reporting magazinc. The share of originl coltilly al contributi finding only a rather limited market (only 337 copics wcre sold in I820) and making losses, subsequently the 'Polytechnische Journal' rapidly developed into one of the most successful magazines in Germany (I724 copies were sold in 1854). This success was to be found in broadly-based 'polytechnical' contents, covering nearly all branches of industry. Another reason for the succes of this masaine was that the subject matter was readily understandable, enhanced by illustrations, and that the most recent foreign and domestic inventions were ro ported on quickly in the 'Polytechnische Journal' Dingler's Technical Journal, in the early industria period, made a considerable contribution to the education of German businessmen and traders, thus promoing the development of industry. The development of this magazine was being investigated until 1855 . The technich technical of the Igth century after the more specialized profession-
al journals began to appear.
Основанный в 1820 году «Политехнический журнал" - старейшее техническое периодическое издание в Германии, возникшее без связи с каким-либо профессиональным объединением. Его основателем и первым издателем был аугсбургский химик й фабрикант Иоганн Готфрид динглер $(1788-1855)$. Журнал выходил в известном «Издательстве классиков» Й. Г. Котта в Штутгарте.

Посте общего введения в үсловия технической информации в начале 19-го века в Германии, в предлагаемом исследовании рассматривается сначала акт основания, программа и личности издателей Политехнижеского журнала. Затем следуют вопросы производства, выпуска и внешнего оформленил, как и проблема издательского тонорара. В соответствии с развитием техники и промьшленного производства, информационный материал заимствовался в первую очередь из английской, затем из французской и лишь в очень постепенно увеличивагощемся обтеме из жемецкой периолической течати, Политехнический журнал был рлавньм образом реферативньм изданием. Процент оригинальных статей всегда оставался небольшим. Посте первоначальных убыттков из-за слабого спроса (в 1820 гопу было продано 337 әкземпляров) Полик(1820 гду било подано з37 экземпллров) Поли作 .

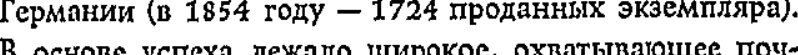

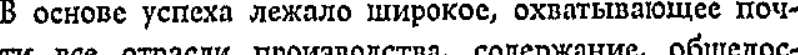

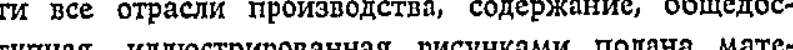
Drence D pen can

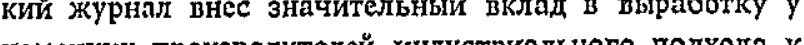
немецких производителей ипдустриального подхода к делу. Разьитие Политехиического журнала рассмптри ваетсл до 1855 года. В середине 19-го века, с появлелием более специализированныт по предмету и профессиональной направленности журналов, времл наибольшего расцвета изданий политехнического характера прошло. 